TRANSACTIONS OF THE

AMERICAN MATHEMATICAL SOCIETY

Volume 364, Number 9, September 2012, Pages 4763-4800

S 0002-9947(2012)05509-7

Article electronically published on April 18, 2012

\title{
WAVE EQUATIONS AND THE LEBRUN-MASON CORRESPONDENCE
}

\author{
FUMINORI NAKATA
}

\begin{abstract}
The LeBrun-Mason twistor correspondences for $S^{1}$-invariant selfdual Zollfrei metrics are explicitly established. The correspondence is described by using explicit formulas for solutions of the wave equation and the monopole equation on the de Sitter three-space, and these formulas are explicitly given by using Radon-type integral transforms. We also obtain a critical condition for the LeBrun-Mason twistor spaces, and show that the twistor correspondence does not work well for twistor spaces which do not satisfy this condition.
\end{abstract}

\section{INTRODUCTION}

The twistor theory concerning holomorphic disks, developed by C. LeBrun and L. J. Mason, is now progressing steadily (see [17, 18, 19, 20, 21, 22, 23, 24]). In general, LeBrun-Mason type twistor correspondence is characterized in the following way:

- the twistor space is given by the pair of a complex manifold $Z$ and a totally real submanifold $P$ in $Z$,

- corresponding objects to the 'twistor lines' in the ordinary twistor theory [1, 11, 26] are given by the holomorphic disks on $Z$ with boundaries lying on $P$,

- a natural differential geometric structure is induced on the parameter space $M$ of the family of holomorphic disks,

- the induced structure is of low regularity in general, and satisfies some global conditions which give a strong restriction on the topology on $M$, and

- conversely, the twistor space $(Z, P)$ is obtained from such a differential geometric structure.

In this article, we mainly deal with the non-rigid case of the LeBrun-Mason correspondence for self-dual conformal structures [19]. In this case, the twistor space is a pair $\left(\mathbb{C P}^{3}, P\right)$ where $P$ is an embedded $\mathbb{R P}^{3}$ sufficiently close to the standard one, and the corresponding geometry is a self-dual indefinite conformal structure $[g]$ on $S^{2} \times S^{2}$ of signature $(--++)$. In this case, the required global condition for $[g]$ is the Zollfrei condition, that is, every maximal null geodesic of $[g]$ is closed (cf. [8]). LeBrun and Mason showed that any self-dual indefinite conformal structure on $S^{2} \times S^{2}$ sufficiently close to the standard one is automatically Zollfrei, and that

Received by the editors January 13, 2010 and, in revised form, October 21, 2010.

2010 Mathematics Subject Classification. Primary 53C28, 35L05, 53C50.

Key words and phrases. Twistor method, holomorphic disks, indefinite metric, wave equation, monopole equation, Radon transform.

This work is partially supported by Research Fellowships of the Japan Society for the Promotion of Science of Young Scientists.

(C)2012 American Mathematical Society Reverts to public domain 28 years from publication 
such conformal structures correspond one-to-one with the twistor spaces $\left(\mathbb{C P}^{3}, P\right)$ in the above sense.

On the other hand, before LeBrun and Mason developed the above theory, infinitely many examples of self-dual indefinite metrics on $S^{2} \times S^{2}$ were obtained by K. P. Tod [27, and independently by H. Kamada [14. Tod constructed $S^{1}$. invariant self-dual indefinite metrics on $S^{2} \times S^{2}$ via a method analogous to what is called LeBrun's hyperbolic ansatz [16. Kamada investigated compact scalar-flat indefinite Kähler surfaces with Hamiltonian $S^{1}$-symmetry. It is known that such a surface is automatically self-dual, and Kamada proved that such a structure is admitted only on $\mathbb{C P}^{1} \times \mathbb{C P}^{1}$. Kamada also constructed infinitely many examples of such structures containing Tod's examples. Since Tod's and Kamada's examples contain the self-dual metrics sufficiently close to the standard one, at least some of them must be Zollfrei by the above results by LeBrun and Mason. So the natural question is the following:

- Are the metrics constructed by Tod and Kamada all Zollfrei?

- If they are Zollfrei, can we establish the LeBrun-Mason correspondences for them?

We show that these problems are settled positively, which is the main theorem in this article (Theorem 7.1).

To attack the above problems, we start from the study of the wave equation and the monopole equation on the three-dimensional de Sitter space $S_{1}^{3}$. We introduce a couple of Radon-type integral transforms from functions on $S^{2}$ to functions on $S_{1}^{3}$, and show that general solutions of the wave equation and the monopole equation satisfying a certain convergence condition are all obtained from functions on $S^{2}$ by making use of the integral transforms. We note that examples of abelian and nonabelian monopoles on $S_{1}^{3}$ are obtained via the twistor method, which was given by V. Kotecha and R. S. Ward [15] before the LeBrun-Mason theory was established. In this article, we deal with abelian and topologically trivial monopoles on $S_{1}^{3}$. We see that all the solutions satisfying the convergency are obtained by the twistor method, and that these solutions are described explicitly and simply by using the introduced integral transforms.

The results in this article are considered as the LeBrun-Mason theory version of the Jones-Tod reduction theory [12]. In contrast, in [22, 23], the author studied the LeBrun-Mason theory version of the Dunajski-West reduction theory [3, 5]. Particularly in 22, we obtain infinitely many self-dual indefinite Zollfrei conformal structures on $S^{2} \times S^{2}$ with singularity, and their LeBrun-Mason correspondences are established by making use of the Radon transform on $\mathbb{R}^{2}$. Though it seems that there are no direct relations between these previous works and the results in this article, these results seem to insist on the significance of the Radon transform as a tool in the study of LeBrun-Mason theory. Here we note that there has been a certain amount of work on the relation between integral geometric transforms and the Penrose transform in the context of split signature twistor correspondences (see, for example, [2]).

The organization of this paper is as follows. We first study the wave equation on the de Sitter space $S_{1}^{3}$ in Sections 2 and 3 . We introduce a couple of Radontype integral transforms, and show that any solution of the wave equation on $S_{1}^{3}$ satisfying a certain convergence condition at infinity is obtained from a function on $S^{2}$ by applying one of the transforms (Theorem 3.1). As a consequence, we see 
that any solution of the wave equation on $S_{1}^{3}$ satisfying the convergency carries a symmetry which we call the oddness.

We next study the monopole equation on $S_{1}^{3}$ in Section 4. We introduce the notion of a monopole potential and show that any gauge equivalent class of monopole solutions corresponds one-to-one with a monopole potential. Further, based on the above results for the wave equation, we show that the gauge equivalent classes of monopole solutions satisfying the convergency correspond one-to-one with functions on $S^{2}$ which we call generating functions (Theorem 4.5).

Following Kamada's formulation, we can construct self-dual indefinite metrics on $S^{2} \times S^{2}$ from monopole solutions on $S_{1}^{3}$ satisfying some extra conditions (Proposition 4.2). In light of this construction, we introduce the notion of admissible monopoles by which we obtain the self-dual metrics on $S^{2} \times S^{2}$ via Kamada's construction. By the above results, the admissible monopoles are obtained from functions on $S^{2}$ satisfying an extra condition corresponding to the admissibility. Tod's and Kamada's examples are obtained by giving examples of an admissible monopole, and in this article we show that their examples essentially cover all the admissible monopoles.

In the latter half of this article (Sections 5 , 6 and 7), we establish the LeBrunMason correspondence for the above-obtained self-dual metrics on $S^{2} \times S^{2}$. We set a twistor space $\left(\mathbb{C P}^{3}, P_{h}\right)$ for each function $h$ on $S^{2}$, and we establish the LeBrunMason correspondence between the twistor space $\left(\mathbb{C P}^{3}, P_{h}\right)$ and the self-dual conformal structure on $S^{2} \times S^{2}$. We see that the self-dual metrics on $S^{2} \times S^{2}$ obtained from admissible monopoles are all Zollfrei. We also study the non-admissible case, and show that the twistor space $\left(\mathbb{C P}^{3}, P_{h}\right)$ carries an unexpected property for holomorphic disks in this case (Proposition 7.3). This means that if the twistor space is non-admissible, the twistor correspondence does not work well at least in the sense so far.

\section{Wave equation on the De Sitter 3-Space}

In this section, we introduce the wave equation on the de Sitter 3-space. Then we introduce integral transforms and show that we can get solutions of the wave equation by these transforms.

The space of small circles. Let $\mathbb{S}^{2}=\left\{\left(u_{1}, u_{2}, u_{3}\right) \in \mathbb{R}^{3} \mid u_{1}^{2}+u_{2}^{2}+u_{3}^{2}=1\right\}$ be the unit sphere equipped with the standard metric and let $\left(S_{1}^{3}, g_{S_{1}^{3}}\right)$ be the de Sitter 3 -space defined by

$$
\begin{aligned}
S_{1}^{3} & :=\left\{\left(x_{0}, x_{1}, x_{2}, x_{3}\right) \in \mathbb{R}^{4} \mid-x_{0}^{2}+x_{1}^{2}+x_{2}^{2}+x_{3}^{2}=1\right\}, \\
g_{S_{1}^{3}} & :=\left.\left(-d x_{0}^{2}+d x_{1}^{2}+d x_{2}^{2}+d x_{3}^{2}\right)\right|_{S_{1}^{3}} .
\end{aligned}
$$

We identify $S_{1}^{3}$ with $\mathbb{R} \times \mathbb{S}^{2}$ via the diffeomorphism $\mathbb{R} \times \mathbb{S}^{2} \stackrel{\sim}{\longrightarrow} S_{1}^{3}$ given by

$$
(t, y) \longmapsto\left(x_{0}, x_{1}, x_{2}, x_{3}\right)=\left(\sinh t, \cosh t\left(y_{1}, y_{2}, y_{3}\right)\right) .
$$

For each $(t, y) \in \mathbb{R} \times \mathbb{S}^{2} \cong S_{1}^{3}$, we define

$$
\Omega_{(t, y)}:=\left\{u \in \mathbb{S}^{2} \mid u \cdot y>\tanh t\right\}
$$

which is an open set on $\mathbb{S}^{2}$ bounded by a small circle. By the correspondence $(t, y) \leftrightarrow \partial \Omega_{(t, y)}$, we identify the de Sitter space $S_{1}^{3}$ with the space of oriented small circles in $\mathbb{S}^{2}$. Notice that the subset $S_{o}^{2}:=\left\{(t, y) \in S_{1}^{3} \mid t=0\right\}$, which is called the neck sphere, corresponds to the space of big circles on $\mathbb{S}^{2}$. Now let us fix $(t, y) \in S_{1}^{3}$ 
and take vectors $y_{1}^{\perp}, y_{2}^{\perp} \in \mathbb{S}^{2}$ so that $\left\{y_{1}^{\perp}, y_{2}^{\perp}, y\right\}$ gives an oriented frame of $\mathbb{R}^{3}$ with compatible orientation. We define a map $\gamma_{(t, y)}: S^{1} \rightarrow \mathbb{S}^{2}$ by

$$
\gamma_{(t, y)}(\phi)=\frac{\cos \phi}{\cosh t} y_{1}^{\perp}+\frac{\sin \phi}{\cosh t} y_{2}^{\perp}+\tanh t y,
$$

which gives a parametrization of the small circle $\partial \Omega_{(t, y)}$. We will see later in Section 6 that the above identification between $S_{1}^{3}$ and the space of the oriented small circles on $\mathbb{S}^{2}$ naturally arises from the LeBrun-Mason correspondence for an Einstein-Weyl 3 -fold.

The wave equation. The wave equation on the de Sitter space $S_{1}^{3}$ is given by

$$
\square V:=* d * d V=0,
$$

where $V$ is a smooth function on $S_{1}^{3}$ and $*$ is the Hodge operator on $S_{1}^{3}$ with respect to the indefinite metric $g_{S_{1}^{3}}$ and the natural orientation on $S_{1}^{3} \simeq \mathbb{R} \times \mathbb{S}^{2}$.

We fix the notation for the operators on $\mathbb{S}^{2}$ as follows: let $\check{*}$ be the Hodge operator, $\check{d}$ be the exterior derivative and $\Delta_{\mathbb{S}^{2}}$ be the Laplace operator. We also use the same notation $\tilde{*}, \breve{d}$ and $\Delta_{\mathbb{S}^{2}}$ for the fiberwise operators on $S_{1}^{3}$ as an $\mathbb{S}^{2}$ bundle $S_{1}^{3}=\mathbb{R} \times \mathbb{S}^{2} \rightarrow \mathbb{R}$. For any one-form $\eta$ on $S_{1}^{3}$ satisfying $\eta\left(\partial_{t}\right)=0$ (where $\left.\partial_{t}=\frac{\partial}{\partial t}\right)$, we obtain

$$
* \eta=-d t \wedge(\check{*} \eta) \quad \text { and } \quad *(d t \wedge \eta)=-* \eta .
$$

If we denote the volume form on $\mathbb{S}^{2}$ by $\omega_{\mathbb{S}^{2}}$, then we have $* d t=-\cosh ^{2} t \omega_{\mathbb{S}^{2}}$. For a smooth function $V$ on $S_{1}^{3}$,

$$
\begin{aligned}
d V & =V_{t} d t+\check{d} V, \\
* d V & =-V_{t} \cosh ^{2} t \omega_{\mathbb{S}^{2}}-d t \wedge(\check{*} \check{d} V), \\
d * d V & =-\left(V_{t} \cosh ^{2} t\right)_{t} d t \wedge \omega_{\mathbb{S}^{2}}+d t \wedge \check{d} \check{*} \check{d} V,
\end{aligned}
$$

where $V_{t}=\partial_{t} V$, and so on. Hence the wave equation (2.2) is written as

$$
\left(-\frac{\partial^{2}}{\partial t^{2}}-2 \tanh t \frac{\partial}{\partial t}+(\cosh t)^{-2} \Delta_{\mathbb{S}^{2}}\right) V=0 .
$$

Function spaces. Let us denote the antipodal map on $\mathbb{S}^{2}$ by $\alpha$. We also define an involution on $S_{1}^{3}$ by $\sigma:(t, y) \mapsto(-t,-y)$. If we identify $S_{1}^{3}$ with the space of oriented small circles on $\mathbb{S}^{2}, \sigma$ corresponds to the orientation-reversing operation for each oriented small circle. Let us denote by $C^{\infty}\left(\mathbb{S}^{2}\right)$ and by $C^{\infty}\left(S_{1}^{3}\right)$ the space of real-valued smooth functions on $\mathbb{S}^{2}$ and on $S_{1}^{3}$ respectively. We set

$$
\begin{aligned}
C_{\text {even }}^{\infty}\left(\mathbb{S}^{2}\right) & :=\left\{h \in C^{\infty}\left(\mathbb{S}^{2}\right) \mid h=h \circ \alpha\right\}, \\
C_{\text {even }}^{\infty}\left(S_{1}^{3}\right) & :=\left\{F \in C^{\infty}\left(S_{1}^{3}\right) \mid F=F \circ \sigma\right\}, \\
C_{\text {odd }}^{\infty}\left(\mathbb{S}^{2}\right) & :=\left\{h \in C^{\infty}\left(\mathbb{S}^{2}\right) \mid h=-h \circ \alpha\right\}, \\
C_{\text {odd }}^{\infty}\left(S_{1}^{3}\right) & :=\left\{F \in C^{\infty}\left(S_{1}^{3}\right) \mid F=-F \circ \sigma\right\} .
\end{aligned}
$$

We call $h \in C_{\text {even }}^{\infty}\left(\mathbb{S}^{2}\right)$ an even function, and so on. We define the maps $\Pi$ : $C^{\infty}\left(\mathbb{S}^{2}\right) \rightarrow \mathbb{R}$ and $\amalg: C^{\infty}\left(S_{1}^{3}\right) \rightarrow \mathbb{R}$ by

$$
\begin{aligned}
\Pi(h) & =\int_{\mathbb{S}^{2}} h \omega_{\mathbb{S}^{2}} \quad \text { for } h \in C^{\infty}\left(\mathbb{S}^{2}\right), \\
\amalg(F) & =\int_{v \in \mathbb{S}^{2}} F(0, v) \omega_{\mathbb{S}^{2}} \quad \text { for } F \in C^{\infty}\left(S_{1}^{3}\right),
\end{aligned}
$$


where $\omega_{\mathbb{S}^{2}}$ is the volume form on $\mathbb{S}^{2}$. We set

$$
\begin{aligned}
C_{*}^{\infty}\left(\mathbb{S}^{2}\right) & :=\left\{h \in C^{\infty}\left(\mathbb{S}^{2}\right) \mid \Pi(h)=0\right\}, \\
C_{*}^{\infty}\left(S_{1}^{3}\right) & :=\left\{F \in C^{\infty}\left(S_{1}^{3}\right) \mid \amalg(F)=0\right\}, \\
C_{\text {even } *}^{\infty}\left(\mathbb{S}^{2}\right) & :=\left\{h \in C_{\text {even }}^{\infty}\left(\mathbb{S}^{2}\right) \mid \Pi(h)=0\right\}, \\
C_{\text {even } *}^{\infty}\left(S_{1}^{3}\right) & :=\left\{F \in C_{\text {even }}^{\infty}\left(S_{1}^{3}\right) \mid \amalg(F)=0\right\} .
\end{aligned}
$$

Let us denote the space of real-valued constant functions by $\mathbb{R}$. Then we obtain the natural decompositions

$C^{\infty}\left(\mathbb{S}^{2}\right)=\mathbb{R} \oplus C_{\text {even } *}^{\infty}\left(\mathbb{S}^{2}\right) \oplus C_{\text {odd }}^{\infty}\left(\mathbb{S}^{2}\right) \quad$ and $\quad C^{\infty}\left(S_{1}^{3}\right)=\mathbb{R} \oplus C_{\text {even } *}^{\infty}\left(S_{1}^{3}\right) \oplus C_{\text {odd }}^{\infty}\left(S_{1}^{3}\right)$ given by $h=\Pi(h)+\left(\frac{1}{2}(h+h \circ \alpha)-\Pi(h)\right)+\frac{1}{2}(h-h \circ \alpha)$, and so on.

Transforms. We define linear transforms $R, Q: C^{\infty}\left(\mathbb{S}^{2}\right) \rightarrow C^{\infty}\left(S_{1}^{3}\right)$ by

$$
\begin{aligned}
R h(t, y) & :=\frac{1}{2 \pi} \int_{0}^{2 \pi} h\left(\gamma_{(t, y)}(\phi)\right) d \phi, \\
Q h(t, y) & :=\frac{1}{2 \pi} \int_{\Omega_{(t, y)}} h(u) \omega_{\mathbb{S}^{2}},
\end{aligned}
$$

where $\gamma_{(t, y)}(\phi)$ is given by (2.1). Of course, $R$ is well-defined by (2.6) without depending on the choice of vectors $\left\{y_{1}^{\perp}, y_{2}^{\perp}\right\}$. By definition we obtain

$$
R\left(C^{\infty}\left(\mathbb{S}^{2}\right)\right) \subset C_{\text {even }}^{\infty}\left(S_{1}^{3}\right), \quad Q\left(C_{*}^{\infty}\left(\mathbb{S}^{2}\right)\right) \subset C_{\text {odd }}^{\infty}\left(S_{1}^{3}\right) .
$$

Restricting $R$ and $Q$ on the neck sphere $S_{o}^{2} \cong \mathbb{S}^{2}$, we also define linear transforms $\mathcal{R}, \mathcal{Q}: C^{\infty}\left(\mathbb{S}^{2}\right) \rightarrow C^{\infty}\left(\mathbb{S}^{2}\right)$ by

$$
\mathcal{R} h(y):=\operatorname{Rh}(0, y) \quad \text { and } \quad \mathcal{Q} h(y):=Q h(0, y) .
$$

The transform $\mathcal{R}$ is called the Funk transform (cf. [6]) or the spherical Radon transform. See [7, 9] for the details of the (spherical) Radon transform and the related topics. The inverse problem for the (spherical) Radon transform is a classical problem, and there are a number of works on this subject. On the other hand, the transform $\mathcal{Q}$ seems to be paid little attention. We will study the inverse problem for the transform $\mathcal{Q}$ in the next section and in Appendix A.

Lemma 2.1. For any smooth function $h$ on $\mathbb{S}^{2}$, the following equation holds:

$$
\frac{\partial}{\partial t} R h(t, y)=-Q \Delta_{\mathbb{S}^{2}} h(t, y) .
$$

Proof. Since we can vary $t$ fixing the frame $\left\{y_{1}^{\perp}, y_{2}^{\perp}, y\right\}$ of $\mathbb{R}^{3}$, we obtain

$$
\frac{\partial}{\partial t}\left(\gamma_{(t, y)}(\phi)\right)=-(\cosh t)^{-1} \nu(\phi),
$$

where $\nu(\phi)$ is the unit normal vector field along $\gamma_{(t, y)}(\phi)$ directed outside of the domain $\Omega_{(t, y)}$. Let $d m$ be the measure on $\partial \Omega_{(t, y)}$ induced by the standard metric on $\mathbb{S}^{2}$. Then we have $d m=(\cosh t)^{-1} d \phi$. Hence we obtain

$$
\frac{\partial}{\partial t} R h(t, y)=-\frac{1}{2 \pi} \int_{\partial \Omega_{(t, y)}}(\nabla h) \cdot \nu d m=-\frac{1}{2 \pi} \int_{\Omega_{(t, y)}}\left(\Delta_{\mathbb{S}^{2}} h\right) \omega_{\mathbb{S}^{2}}=-Q \Delta_{\mathbb{S}^{2}} h(t, y)
$$

by the divergence formula. 
Lemma 2.2. For any smooth function $h$ on $\mathbb{S}^{2}$, the following equation holds:

$$
\frac{\partial}{\partial t} Q h(t, y)=-(\cosh t)^{-2} R h(t, y) \text {. }
$$

Proof. We fix $y \in \mathbb{S}^{2}$ and take vectors $y_{1}^{\perp}, y_{2}^{\perp} \in \mathbb{S}^{2}$ so that $\left\{y_{1}^{\perp}, y_{2}^{\perp}, y\right\}$ gives an oriented orthonormal basis on $\mathbb{R}^{3}$. We use a spherical coordinate $(\theta, \phi) \in(0, \pi) \times$ $(0,2 \pi)$ on $\mathbb{S}^{2}$ defined by

$$
(\theta, \phi) \longmapsto u(\theta, \phi):=\sin \theta \cos \phi y_{1}^{\perp}+\sin \theta \sin \phi y_{2}^{\perp}+\cos \theta y .
$$

Then we have $\Omega_{(t, y)}=\left\{(\theta, \phi) \in \mathbb{S}^{2} \mid 0 \leq \theta \leq \alpha\right\}$, where $\alpha$ is the real variable defined by $\cos \alpha=\tanh t$. In this coordinate, noticing $\omega_{\mathbb{S}^{2}}=\sin \theta d \theta \wedge d \phi$,

$$
Q h(t, y)=\int_{\Omega_{(t, y)}} h(u(\theta, \phi)) \sin \theta d \theta \wedge d \phi=\int_{0}^{2 \pi}\left[\int_{0}^{\alpha} h(u(\theta, \phi)) \sin \theta d \theta\right] d \phi .
$$

Since $\frac{\partial}{\partial t}=-(\cosh t)^{-1} \frac{\partial}{\partial \alpha}$, we obtain

$$
\begin{aligned}
\frac{\partial}{\partial t} Q h(t, y) & =\frac{-1}{\cosh t} \int_{0}^{2 \pi} h(u(\alpha, \phi)) \sin \alpha d \phi \\
& =\frac{-1}{\cosh ^{2} t} \int_{0}^{2 \pi} h(u(\alpha, \phi)) d \phi=-\frac{R h(t, y)}{\cosh ^{2} t}
\end{aligned}
$$

as required.

Remark 2.3. We can check $Q(1)(t, y)=\operatorname{Area}\left(\Omega_{(t, y)}\right)=1-\tanh t$ by using the above coordinate $(\theta, \phi)$.

Proposition 2.4. For any smooth function $h$ on $\mathbb{S}^{2}$, the induced function $f:=R h$ on $S_{1}^{3}$ solves the following hyperbolic partial differential equation:

$$
L f:=\left(-\frac{\partial^{2}}{\partial t^{2}}+(\cosh t)^{-2} \Delta_{\mathbb{S}^{2}}\right) f=0 .
$$

Proof. First we claim that $R$ commutes with $\Delta_{\mathbb{S}^{2}}$. Actually, if we fix $t \in \mathbb{R}$, the transform $M^{t}: h \longmapsto R h(t, \cdot)$ is $S O(3)$-equivariant; hence $M^{t}$ commutes with $\Delta_{\mathbb{S}^{2}}$ by Theorem A.1 in Appendix A. Thus $R$ commutes with $\Delta_{\mathbb{S}^{2}}$. Then, by the above lemmas,

$$
\frac{\partial^{2}}{\partial t^{2}} R h(t, y)=-\frac{\partial}{\partial t} Q \Delta_{\mathbb{S}^{2}} h(t, y)=(\cosh t)^{-2} R \Delta_{\mathbb{S}^{2}} h(t, y)=(\cosh t)^{-2} \Delta_{\mathbb{S}^{2}} R h(t, y)
$$

for any smooth function $h$ on $\mathbb{S}^{2}$. Hence $f:=R h$ solves (2.9).

Lemma 2.5. Let $f$ be a smooth function on $S_{1}^{3}$ satisfying the equation $L f=0$. If we put $V:=f_{t}$, then $V$ satisfies the wave equation $\square V=0$.

Proof. Applying $\frac{\partial}{\partial t}$ on the equation $(\cosh t)^{2} L f=0$, we obtain the equation (2.5).

Proposition 2.6. Let $h$ be a smooth function on $\mathbb{S}^{2}$ satisfying $\Pi(h)=0$. Then $V:=Q h$ solves the wave equation $\square V=0$.

Proof. Since $\Pi(h)=0$, there exists a smooth function $\tilde{h}$ on $\mathbb{S}^{2}$ satisfying $h=-\Delta_{\mathbb{S}^{2}} \tilde{h}$. If we put $f:=R \tilde{h}$, then $V=-Q \Delta_{\mathbb{S}^{2}} \tilde{h}=f_{t}$ by Lemma 2.1. On the other hand, $L f=0$ by Proposition 2.4 so $\square V=\square f_{t}=0$ by Lemma 2.5.

Remark 2.7. We call a function $h \in C_{*}^{\infty}\left(\mathbb{S}^{2}\right)$ a generating function in the sense that $h$ induces a solution of $\square V=0$ or $L f=0$. 


\section{OdDNESS AND THE INVERSE PROBLEM}

In this section, we investigate the inverse problem for the transforms $R$ and $Q$. The goal is the following.

Theorem 3.1. (1) Let $V$ be a smooth function on $S_{1}^{3}$ which solves the wave equation $\square V=0$. Suppose that $V \rightarrow 0$ and $V_{t} \rightarrow 0$ as $t \rightarrow \pm \infty$ uniformly for $y \in \mathbb{S}^{2}$. Then $V$ is odd, and there exists a unique smooth function $h \in C_{*}^{\infty}\left(\mathbb{S}^{2}\right)$ satisfying $V=Q h$.

(2) Let $f$ be a smooth function on $S_{1}^{3}$ which solves the equation $L f=0$. Suppose that there exist $h_{ \pm}(y) \in C^{\infty}\left(\mathbb{S}^{2}\right)$ and that $f(t, y) \rightarrow h_{ \pm}(y)$ and $f_{t}, f_{t t} \rightarrow 0$ as $t \rightarrow \pm \infty$ uniformly for $y \in \mathbb{S}^{2}$. Then $f$ is even, and $f=R h_{+}$holds. Moreover, if $f \in C_{*}^{\infty}\left(S_{1}^{3}\right)$, then $h_{ \pm} \in C_{*}^{\infty}\left(\mathbb{S}^{2}\right)$.

Inverse problem for $\mathcal{R}$ and $\mathcal{Q}$. First, we study the transforms $\mathcal{R}$ and $\mathcal{Q}$. By definition, $\mathcal{R}$ and $\mathcal{Q}$ are the identities on the constant functions $\mathbb{R} \subset C^{\infty}\left(\mathbb{S}^{2}\right)$. We also have $\mathcal{R}\left(C_{\text {odd }}^{\infty}\left(\mathbb{S}^{2}\right)\right)=\mathcal{Q}\left(C_{\text {even } *}^{\infty}\left(\mathbb{S}^{2}\right)\right)=0$. The following bijectivity is, however, rather non-trivial.

Proposition 3.2. Both of the following transforms are bijective:

(1) $\mathcal{R}: C_{\text {even } *}^{\infty}\left(\mathbb{S}^{2}\right) \longrightarrow C_{\text {even* }}^{\infty}\left(\mathbb{S}^{2}\right)$,

(2) $\mathcal{Q}: C_{\text {odd }}^{\infty}\left(\mathbb{S}^{2}\right) \longrightarrow C_{\text {odd }}^{\infty}\left(\mathbb{S}^{2}\right)$.

Hence we obtain

$$
\begin{gathered}
\operatorname{ker}\left\{\mathcal{R}: C^{\infty}\left(\mathbb{S}^{2}\right) \rightarrow C^{\infty}\left(\mathbb{S}^{2}\right)\right\}=C_{\text {odd }}^{\infty}\left(\mathbb{S}^{2}\right), \\
\operatorname{ker}\left\{\mathcal{Q}: C^{\infty}\left(\mathbb{S}^{2}\right) \rightarrow C^{\infty}\left(\mathbb{S}^{2}\right)\right\}=C_{\text {even } *}^{\infty}\left(\mathbb{S}^{2}\right)
\end{gathered}
$$

The above bijectivity of $\mathcal{R}$ on $C_{\text {even } *}^{\infty}\left(\mathbb{S}^{2}\right)$ was first noticed by P. Funk [6]. There is an explicit inversion formula of $\mathcal{R}$ or its generalization, which we can find in the textbook by S. Helgason [9]. On the other hand, just for the purpose of verifying the bijectivity of $\mathcal{R}, \mathrm{V}$. Guillemin's method is reasonable (Appendix A of [7]). We give the proof of the bijectivity of $\mathcal{Q}$ on $C_{\text {odd }}^{\infty}\left(\mathbb{S}^{2}\right)$ in Appendix A by a similar argument as Guillemin's.

Key lemma. The key to proving Theorem 3.1 is to verify the oddness and evenness for the initial values $\left.V\right|_{t=0}$ and $\left.V_{t}\right|_{t=0}$, which will be shown in Lemma 3.4. Before this, we first notice the following.

Lemma 3.3. For each function $V \in C^{\infty}\left(S_{1}^{3}\right)$, we define a function $I(\tau)$ on $\tau \in \mathbb{R}$ by

$$
I(\tau):=\frac{\cosh ^{2} \tau}{2 \pi} \int_{\mathbb{S}^{2}}\left(\left.V_{t}\right|_{t=\tau}\right) \omega_{\mathbb{S}^{2}}
$$

If $V$ solves the wave equation $\square V=0$, then $I(\tau)$ is independent with $\tau \in \mathbb{R}$.

Proof. Let $p: S_{1}^{3}=\mathbb{R} \times \mathbb{S}^{2} \rightarrow \mathbb{R}$ be the projection, and let $t_{1}$ and $t_{2}$ be real numbers such that $t_{1}<t_{x}$. If $V$ satisfies $\square V=* d * d V=0$, then we obtain

$$
0=\frac{1}{2 \pi} \int_{p^{-1}\left(t_{1}, t_{2}\right)} d * d V=\frac{-1}{2 \pi} \int_{p^{-1}\left(t_{2}\right)-p^{-1}\left(t_{1}\right)} V_{t} \cosh ^{2} t \omega_{\mathbb{S}^{2}}=-I\left(t_{2}\right)+I\left(t_{1}\right) .
$$

Hence $I(\tau)$ does not depend on $\tau \in \mathbb{R}$.

Lemma 3.4. Let $V$ be a smooth function on $S_{1}^{3}$ which solves the wave equation $\square V=0$. Suppose that $V \rightarrow 0$ and $V_{t} \rightarrow 0$ as $t \rightarrow \pm \infty$ uniformly for $y \in \mathbb{S}^{2}$. Let $\psi(y):=V(0, y)$ and $\xi(y):=V_{t}(0, y)$. Then $\psi \in C_{\text {odd }}^{\infty}\left(\mathbb{S}^{2}\right)$ and $\xi \in C_{\text {even } *}^{\infty}\left(\mathbb{S}^{2}\right)$. 
Proof. We fix $y \in \mathbb{S}^{2}$ and take a coordinate $(\theta, \phi)$ on $\mathbb{S}^{2}$ similarly as in the proof of Lemma 2.2. We use the coordinate $(t, \theta, \phi) \in \mathbb{R} \times \mathbb{S}^{2} \cong S_{1}^{3}$, and we put

$$
\begin{aligned}
\Omega_{(t, y)}^{\prime} & :=\{t\} \times \Omega_{(t, v)} \subset S_{1}^{3}, \\
M_{y}\left(t_{1}, t_{2}\right) & :=\left\{(t, \theta, \phi) \in S_{1}^{3} \mid t_{1}<t<t_{2}, \cos \theta>\tanh t\right\}=\bigcup_{t \in\left(t_{1}, t_{2}\right)} \Omega_{(t, y)}^{\prime}, \\
\Sigma_{y}\left(t_{1}, t_{2}\right) & :=\left\{(t, \theta, \phi) \in S_{1}^{3} \mid t_{1}<t<t_{2}, \cos \theta=\tanh t\right\}=\bigcup_{t \in\left(t_{1}, t_{2}\right)} \partial \Omega_{(t, y)}^{\prime} .
\end{aligned}
$$

Notice that

$$
\begin{aligned}
\partial M_{y}\left(t_{1}, t_{2}\right) & =\Sigma_{y}\left(t_{1}, t_{2}\right) \cup \Omega_{\left(t_{2}, y\right)}^{\prime} \cup\left(-\Omega_{\left(t_{1}, y\right)}^{\prime}\right), \\
\partial \Sigma_{y}\left(t_{1}, t_{2}\right) & =\partial \Omega_{\left(t_{2}, y\right)}^{\prime} \cup\left(-\partial \Omega_{\left(t_{1}, y\right)}^{\prime}\right) .
\end{aligned}
$$

Now let $V$ be a function on $S_{1}^{3}$ as in the statement and $\tau$ be a positive real variable. Since $\square V=* d * d V=0$, integrating on $M_{y}\left(t_{1}, t_{2}\right)$, we obtain

$$
0=\int_{M_{y}\left(t_{1}, t_{2}\right)} d * d V=\int_{\Sigma_{y}\left(t_{1}, t_{2}\right)+\Omega_{\left(t_{2}, y\right)}^{\prime}-\Omega_{\left(t_{1}, y\right)}^{\prime}} * d V .
$$

For $i=1,2$, let $\alpha_{i} \in(0, \pi)$ be the real variable defined by $\cos \alpha_{i}=\tanh t_{i}$. To calculate the integral over $\Sigma_{y}\left(t_{1}, t_{2}\right)$, we introduce a real coordinate $(a, b) \in$ $\left(\alpha_{2}, \alpha_{1}\right) \times(0,2 \pi)$ on $\Sigma_{y}\left(t_{1}, t_{2}\right)$ by the embedding $j:\left(\alpha_{2}, \alpha_{1}\right) \times(0,2 \pi) \rightarrow \Sigma_{y}\left(t_{1}, t_{2}\right)$ defined by $(t, \theta, \phi)=(t(a), a, b)$, where $\cos a=\tanh t(a)$. Then we obtain

$$
\begin{aligned}
* d V & =-V_{t} \cosh ^{2} t \omega_{\mathbb{S}^{2}}-d t \wedge \check{*} \check{d} V=\left(-(\sin a)^{-1} V_{t}+V_{\theta}\right) d a \wedge d b=-\frac{\partial V}{\partial a} d a \wedge d b \\
& =-d(V d b) .
\end{aligned}
$$

Hence

$$
\begin{aligned}
\frac{1}{2 \pi} \int_{\Sigma_{y}\left(t_{1}, t_{2}\right)} * d V & =\frac{-1}{2 \pi} \int_{\Sigma_{y}\left(t_{1}, t_{2}\right)} d(V d b)=\frac{-1}{2 \pi} \int_{\partial \Omega_{\left(t_{2}, y\right)}^{\prime}-\partial \Omega_{\left(t_{1}, y\right)}^{\prime}} V d \phi \\
& =-R\left(\left.V\right|_{t=t_{2}}\right)\left(t_{2}, y\right)+R\left(\left.V\right|_{t=t_{1}}\right)\left(t_{1}, y\right) .
\end{aligned}
$$

On the other hand, for each $\tau \in \mathbb{R}$ we have

$$
\frac{1}{2 \pi} \int_{\Omega_{(\tau, y)}^{\prime}} * d V=\frac{-1}{2 \pi} \int_{\Omega_{(\tau, y)}^{\prime}} V_{t} \cosh ^{2} t \omega_{\mathbb{S}^{2}}=-\cosh ^{2} \tau Q\left(\left.V_{t}\right|_{t=\tau}\right)(\tau, y) .
$$

Hence by (3.2), we see that the quantity

$$
E(y):=R\left(\left.V\right|_{t=\tau}\right)(\tau, y)+\cosh ^{2} \tau Q\left(\left.V_{t}\right|_{t=\tau}\right)(\tau, y)
$$

does not depend on $\tau \in \mathbb{R}$.

Now we claim $E(y) \equiv 0$. Notice that

$$
\cosh ^{2} \tau\left|\int_{\Omega_{(\tau, y)}^{\prime}} V_{t} \omega_{\mathbb{S}^{2}}\right| \leq \cosh ^{2} \tau \operatorname{Area}\left(\Omega_{(\tau, y)}\right) \cdot \max _{u \in \Omega_{(\tau, y)}^{\prime}}\left|V_{t}(u)\right| \leq \max _{u \in \Omega_{(\tau, y)}^{\prime}}\left|V_{t}(u)\right|
$$

since $\operatorname{Area}\left(\Omega_{(\tau, y)}\right)=1-\tanh \tau$. Hence we obtain $\lim _{\tau \rightarrow+\infty}\left[\cosh ^{2} \tau Q\left(\left.V_{t}\right|_{t=\tau}\right)(\tau, y)\right]$ $=0$ by the convergence of $V_{t}$. On the other hand, by the convergence of $V$, we also have $\lim _{\tau \rightarrow \pm \infty}\left[R\left(\left.V\right|_{t=\tau}\right)(\tau, y)\right]=0$. Thus, by taking the limit $\tau \rightarrow+\infty$ on (3.3), we obtain $E(y) \equiv 0$ as required. 
Next notice that

$$
I=\cosh ^{2} \tau Q\left(\left.V_{t}\right|_{t=\tau}\right)(\tau, y)+\cosh ^{2} \tau Q\left(\left.V_{t}\right|_{t=\tau}\right)(-\tau,-y)
$$

where $I$ is the quantity defined in (3.1). If we take the limit $\tau \rightarrow-\infty$, then the second term of the right-hand side of (3.5) vanishes by an argument similar to the above. Hence we obtain

$$
I=\lim _{\tau \rightarrow-\infty}\left[\cosh ^{2} \tau Q\left(\left.V_{t}\right|_{t=\tau}\right)(\tau, y)\right] .
$$

Thus, by taking the limit $\tau \rightarrow-\infty$ on (3.3), we obtain $I=0$. This means $\left.V_{t}\right|_{t=\tau} \in$ $C_{*}^{\infty}\left(\mathbb{S}^{2}\right)$ for any $\tau \in \mathbb{R}$.

Finally, evaluating $\tau=0$ to (3.3), we obtain $\mathcal{R} \psi+\mathcal{Q} \xi=0$. Recall that $\mathcal{R} \psi \in$ $C_{\text {even }}^{\infty}\left(\mathbb{S}^{2}\right)$ and $\mathcal{Q} \xi \in \mathbb{R} \oplus C_{\text {odd }}^{\infty}\left(\mathbb{S}^{2}\right)$ by Proposition 3.2. Further, since $\xi=\left.V_{t}\right|_{t=0} \in$ $C_{*}^{\infty}\left(\mathbb{S}^{2}\right)$ by the above argument, we have $\mathcal{Q} \xi \in C_{\text {odd }}^{\infty}\left(\mathbb{S}^{2}\right)$. Thus we obtain $\mathcal{R} \psi=$ $\mathcal{Q} \xi=0$. Hence $\psi \in C_{\text {odd }}^{\infty}\left(\mathbb{S}^{2}\right)$ and $\xi \in C_{\text {even } *}^{\infty}\left(\mathbb{S}^{2}\right)$ by Proposition 3.2 .

Proof of Theorem [3.1. Let $V$ be as in statement $(1)$, and let $\psi(y):=V(0, y)$ and $\xi(y):=V_{t}(0, y)$. By Lemma 3.4, we have $\psi \in C_{\text {odd }}^{\infty}\left(\mathbb{S}^{2}\right)$ and $\xi \in C_{\text {even } *}^{\infty}\left(\mathbb{S}^{2}\right)$. Then by Proposition 3.2. there exist smooth functions $h_{\text {odd }} \in C_{\text {odd }}^{\infty}\left(\mathbb{S}^{2}\right)$ and $h_{\text {even }} \in$ $C_{\text {even } *}^{\infty}\left(\mathbb{S}^{2}\right)$ satisfying

$$
\psi=\mathcal{Q} h_{\text {odd }}, \quad \xi=-\mathcal{R} h_{\text {even }} .
$$

Now let us put $h:=h_{\text {even }}+h_{\text {odd }}$ and $\tilde{V}:=Q h$. Since $h \in C_{*}^{\infty}\left(\mathbb{S}^{2}\right), \tilde{V}$ is a solution of $\square \tilde{V}=0$ by Proposition 2.6. Moreover by construction

$$
\tilde{V}(0, y)=\mathcal{Q} h(y)=\psi(y), \quad \tilde{V}_{t}(0, y)=\left[\frac{\partial}{\partial t} Q h(t, y)\right]_{t=0}=-\mathcal{R} h(y)=\xi(y) .
$$

Hence $V$ and $\tilde{V}$ satisfy the same initial condition, so by the uniqueness theorem for the initial value problem of hyperbolic partial differential equations (see [4]), we obtain $V=\tilde{V}$. Hence $V=Q h$ and $V$ turns out to be odd. The uniqueness of $h$ is obvious by relation (3.6).

Next let $f$ be as in statement (2). If we put $V:=f_{t}$, then $V$ satisfies the conditions in statement (1). Hence $V$ is odd. If we decompose $f$ as $f=f_{\text {even }}+f_{\text {odd }}$ so that $f_{\text {even }} \in C_{\text {even }}^{\infty}\left(S_{1}^{3}\right)$ and $f_{\text {odd }} \in C_{\text {odd }}^{\infty}\left(S_{1}^{3}\right)$, then $V_{t}=f_{t}=\left(f_{\text {odd }}\right)_{t}+\left(f_{\text {even }}\right)_{t}$ gives the decomposition of $V$ satisfying $\left(f_{\text {odd }}\right)_{t} \in C_{\text {even }}^{\infty}\left(S_{1}^{3}\right)$ and $\left(f_{\text {even }}\right)_{t} \in C_{\text {odd }}^{\infty}\left(S_{1}^{3}\right)$. Since $V$ is odd, we obtain $\left(f_{\text {odd }}\right)_{t}=0$. Hence $f_{\text {odd }}=0$ and $f$ is even.

Let us put $\varphi(y):=f(0, y)$ and $\psi(y):=f_{t}(0, y)$. Then, similar to the above argument, there is a unique smooth function $\tilde{h}$ on $\mathbb{S}^{2}$ which satisfies

$$
\varphi(y)=\mathcal{R} \tilde{h}(y), \quad \psi(y)=-\mathcal{Q} \Delta_{\mathbb{S}^{2}} \tilde{h}(y) .
$$

For this function $\tilde{h}$, we obtain $f=R \tilde{h}$. By the definition of $R$,

$$
h_{+}(y)=\lim _{t \rightarrow \infty} f(t, y)=\lim _{t \rightarrow \infty} R \tilde{h}(t, y)=\tilde{h}(y) .
$$

Hence $f=R h_{+}$as required. If $f \in C_{*}^{\infty}\left(S_{1}^{3}\right)$, then $\varphi \in C_{\text {even } *}^{\infty}\left(\mathbb{S}^{2}\right)$ and we obtain $h_{+}=\tilde{h} \in C_{*}^{\infty}\left(\mathbb{S}^{2}\right)$ by the construction. Since $f$ is even, $h_{-}(y)=h_{+}(-y) \in$ $C_{*}^{\infty}\left(\mathbb{S}^{2}\right)$.

Convergency at infinity. By Theorem 3.1 and its proof, we can paraphrase the condition of the convergency at infinity for $V$ in the following way. 
Corollary 3.5. Let $V \in C^{\infty}\left(S_{1}^{3}\right)$ be a solution of the wave equation $\square V=0$. Then the following conditions are equivalent:

(1) $V(t, y) \rightarrow 0$ and $V_{t}(t, y) \rightarrow 0$ as $t \rightarrow \pm \infty$ uniformly for $y \in \mathbb{S}^{2}$,

(2) $V$ is odd and $I=0$, and

(3) $\psi(y):=V(0, y) \in C_{\text {odd }}^{\infty}\left(\mathbb{S}^{2}\right)$ and $\xi(y):=V_{t}(0, y) \in C_{\text {even } *}^{\infty}\left(\mathbb{S}^{2}\right)$.

Proof. Statement $(1) \Rightarrow(2)$ follows from Theorem 3.1, and (2) $\Rightarrow(3)$ is obvious. Now let us assume (3). As in the proof of Theorem 3.1, we have $V=Q h$ for $h=h_{\text {even }}+h_{\text {odd }}$ where $h_{\text {even }} \in C_{\text {even } *}^{\infty}\left(\mathbb{S}^{2}\right)$ and $h_{\text {odd }} \in C_{\text {odd }}^{\infty}\left(\mathbb{S}^{2}\right)$ are defined by (3.6). Then we can check that $V=Q h$ and $V_{t}=-(\cosh t)^{-2} R h$ uniformly converge to zero as $t \rightarrow \pm \infty$. Thus (3) $\Rightarrow(1)$ holds.

Similarly, we obtain the following result of which the proof is omitted.

Corollary 3.6. Let $f \in C^{\infty}\left(S_{1}^{3}\right)$ be a solution of the equation $L f=0$. Then the following conditions are equivalent:

(1) There exist smooth functions $h_{ \pm}(y) \in C^{\infty}\left(\mathbb{S}^{2}\right)$ such that $f(t, y) \rightarrow h_{ \pm}(y)$ and $f_{t}, f_{t t} \rightarrow 0$ as $t \rightarrow \pm \infty$ uniformly for $y \in \mathbb{S}^{2}$,

(2) $f$ is even, and

(3) $\varphi(y):=f(0, y)$ is even and $\psi(y):=f_{t}(0, y)$ is odd.

Rigidity theorem. Let $S_{1}^{3} / \mathbb{Z}_{2}$ be the quotient space of $S_{1}^{3}$ by the involution $\sigma$. Notice that $S_{1}^{3} / \mathbb{Z}_{2}$ is not space-time orientable. Since the operator $\square$ on $S_{1}^{3}$ is $\sigma$-invariant, we can define the wave equation $\square V=0$ on $S_{1}^{3} / \mathbb{Z}_{2}$. Now let us use the coordinate $\left\{(t, y) \in \mathbb{R} \times \mathbb{S}^{2} \mid t>0\right\}$ on the open set $\left\{[t, y] \in S_{1}^{3} / \mathbb{Z}_{2} \mid t \neq 0\right\}$. Then, as a trivial consequence of Theorem 3.1, we obtain the following rigidity theorem.

Corollary 3.7. Let $V$ be a solution of the wave equation $\square V=0$ on $S_{1}^{3} / \mathbb{Z}_{2}$. Suppose $V, V_{t} \rightarrow 0$ as $t \rightarrow \infty$ uniformly for $y$. Then $V \equiv 0$.

We remark that this type of rigidity theorem is also found in [18] or [19]. For example in [19, it is shown that the standard self-dual indefinite metric on the nonspace-time orientable space $\left(S^{2} \times S^{2}\right) / \mathbb{Z}_{2}$ is rigid in the space of self-dual metrics.

Relation with representation theory. The de Sitter space $S_{1}^{3}$ can be dealt with as a homogeneous space, and the wave equation on $S_{1}^{3}$ is a homogeneous equation, so it is reasonable to investigate the wave equation on $S_{1}^{3}$ from a representationtheoretical viewpoint. Actually, such an investigation is given in a quite general setting (see [25]), and the cases for hyperboloids containing $S_{1}^{3}$ are studied in detail [10. Though the above result in this article is concerned with a very special homogeneous space $S_{1}^{3}$, our result has a rather different flavor from these previous works on representation theory. Indeed, our result is based on twistor theory, the operator $Q$ is newly noticed and it enables us to describe the solutions quite simply, and generalizations to non-homogeneous cases are naturally expected as a future perspective of our theory.

\section{Monopole EQuation}

In this section, we investigate the monopole equation over the de Sitter space $S_{1}^{3}$. We show that any gauge equivalence class of monopole solutions is obtained from a solution of $L f=0$ which we call the monopole potential. Then, applying Theorem 3.1. we establish a one-to-one correspondence between generating functions $h \in$ $C_{*}^{\infty}\left(\mathbb{S}^{2}\right)$ and gauge equivalent classes of monopole solutions on $S_{1}^{3}$ which satisfy a 
convergence condition at infinity. Further, we introduce the notion of admissible monopoles by which we can construct $S^{1}$-invariant self-dual metrics on $S^{2} \times S^{2}$.

Tod-Kamada ansatz. Here we review the construction of self-dual metrics on $S^{2} \times S^{2}$ given by Tod and Kamada, following Kamada's formulation.

The basic construction is the following.

Proposition 4.1 (Kamada 14). Let $V$ be a smooth positive function on $S_{1}^{3}$ such that $* d V / 2 \pi$ is a closed two-form on $S_{1}^{3}$ determining an integral class in $H^{2}\left(S_{1}^{3} ; \mathbb{R}\right)$. Let $\mathcal{M} \rightarrow S_{1}^{3}$ denote an $S^{1}$-bundle with connection one-form $\Theta$ with curvature form given by

$$
d \Theta=* d V .
$$

Then $g_{V, \Theta}:=-V^{-1} \Theta \otimes \Theta+V g_{S_{1}^{3}}$ is a self-dual metric on $\mathcal{M}$ of signature $(--++)$ with respect to a suitable orientation on $\mathcal{M}$.

Now we study the case when $* d V$ is exact, i.e. when the $S^{1}$-bundle $\mathcal{M} \rightarrow S_{1}^{3}$ is trivial. In this case, we write $\mathcal{M} \simeq S^{1} \times S_{1}^{3}=\left\{(s, t, y) \in S^{1} \times \mathbb{R} \times \mathbb{S}^{2}\right\}$, where $s$ is the fiber coordinate and $S_{1}^{3}=\left\{(t, y) \in \mathbb{R} \times \mathbb{S}^{2}\right\}$. The total space $\mathcal{M}$ is naturally compactified to $\overline{\mathcal{M}}:=S^{2} \times S^{2}$ by the embedding $\mathcal{M} \hookrightarrow S^{2} \times S^{2}:(s, t, y) \mapsto(x, y)$, where

$$
x^{1}=\frac{\cos s}{\cosh t}, \quad x^{2}=\frac{\sin s}{\cosh t}, \quad x^{3}=\tanh t
$$

In other words, $\mathcal{M}$ is obtained as the free part of the $S^{1}$-action on $S^{2} \times S^{2}$ defined by

$$
\alpha \cdot(x, y)=(R(\alpha) x, y), \quad \text { where } \alpha \in S^{1} \text { and } R(\alpha)=\left(\begin{array}{ccc}
\cos \alpha & -\sin \alpha & 0 \\
\sin \alpha & \cos \alpha & 0 \\
0 & 0 & 1
\end{array}\right) \text {. }
$$

If we put $\varepsilon:=(0,0,1) \in S^{2}$ and $S_{ \pm}:=\{ \pm \varepsilon\} \times S^{2} \subset S^{2} \times S^{2}$, then the disjoint union $S_{+} \sqcup S_{-}$coincides to the fixed point set of the above $S^{1}$-action, and we have $\mathcal{M}=\left(S^{2} \times S^{2}\right) \backslash\left(S_{+} \sqcup S_{-}\right)$. Let us introduce variables $r:=e^{t}$ and $q:=e^{-t}$. Then $(s, r)$ and $(s, q)$ give the polar coordinates on the open neighborhoods of $-\varepsilon \in S^{2}$ and $\varepsilon \in S^{2}$ respectively.

Proposition 4.2 (Kamada 14). Let $(V, \Theta)$ be a smooth solution of (4.1) such that $V>0$ and $* d V$ is an exact two-form. Then the metric $\bar{g}_{V, \Theta}:=(\cosh t)^{-2} g_{V, \Theta}$ on $\mathcal{M}$ extends smoothly to the compactification $\overline{\mathcal{M}}=S^{2} \times S^{2}$ if and only if there exist smooth functions $F_{+}$and $F_{-}$on $\mathbb{R} \times \mathbb{S}^{2}$ in variables $r^{2}, q^{2}$ and $y$ such that

$$
V=1+r^{2} F_{-}\left(r^{2}, y\right) \text { and } V=1+q^{2} F_{+}\left(q^{2}, y\right),
$$

as $r \rightarrow+0$ and as $q \rightarrow+0$ respectively.

If $* d V$ is exact, $\Theta$ is written as $\Theta=d s+A$ using a one-form $A$ on $S_{1}^{3}$. Then the equation (4.1) is written as

$$
d A=* d V
$$

which we call the monopole equation. We call a solution $(V, A)$ of (4.5) a monopole solution or simply a monopole. We write $\bar{g}_{V, A}=\bar{g}_{V, \Theta}$, where $\Theta=d s+A$, and we also use the notation $\bar{g}_{V, A}$ for its compactification. Notice that if $(V, A)$ is a monopole, then $V$ satisfies the wave equation $\square V=* d * d V=0$.

The simplest solution of the monopole equation satisfying the condition (4.4) is given by $(V, A)=(1,0)$, which we call the trivial monopole. In this case, the 
self-dual indefinite metric induced on $S^{2} \times S^{2}$ is the standard indefinite metric, i.e. the product metric $g_{0}=\pi_{1}^{*} h-\pi_{2}^{*} h$, where $\pi_{i}: S^{2} \times S^{2} \rightarrow S^{2}$ is the $i$-th projection and $h$ is the standard metric on $S^{2}$.

Tod's or Kamada's examples of self-dual indefinite metrics are obtained by constructing explicit solutions of (4.5). We deal with these examples in the last part of this section.

Monopole potential. Now we show that any solution of the monopole equation (4.5) essentially arises from a function $f \in C_{*}^{\infty}\left(S_{1}^{3}\right)$ satisfying $L f=0$, where $L$ is the partial differential operator defined in (2.9). We call such an $f$ the monopole potential.

For each real-valued function $\phi \in C^{\infty}\left(S_{1}^{3}\right)$, the transform of monopoles

$$
(V, A) \longmapsto(V, A+d \phi)
$$

is called the gauge transform. Notice that $\Phi^{*} \bar{g}_{V, A}=\bar{g}_{V, A+d \phi}$, where $\Phi=e^{i \phi}: \mathcal{M} \rightarrow$ $\mathcal{M}$ is the gauge transform on the $S^{1}$-bundle $\mathcal{M} \rightarrow S_{1}^{3}$.

Proposition 4.3. Let $(V, A)$ be any monopole on $S_{1}^{3}$. Then, changing $(V, A)$ by a gauge transform, we can assume $\left(1^{\circ}\right) A\left(\partial_{t}\right)=0$ and $\left(2^{\circ}\right) \check{d} \check{*} A=0$, where $\breve{d}$ is the fiberwise exterior derivative and $\tilde{*}$ is the fiberwise Hodge operator on the $\mathbb{S}^{2}$-bundle $S_{1}^{3} \rightarrow \mathbb{R}$. Furthermore such a $(V, A)$ is unique in the gauge equivalence class.

Proof. Let $(V, A)$ be any monopole on $S_{1}^{3}$. Let us write $A=A_{t} d t+A_{1}$ so that $A_{1}$ is a one-form without $d t$-part. If we put $\phi_{1}=-\int_{0}^{t} A_{t} d t$, then the one-form $A+d \phi_{1}$ does not have a $d t$-part. Hence we can assume that $A$ satisfies the condition $\left(1^{\circ}\right)$ from the beginning.

By the monopole equation (4.5), $A=A_{1}$ satisfies $d * d A=0$. Since $d A=$ $d t \wedge \frac{\partial A}{\partial t}+\check{d} A$, we can write

$$
0=d * d A \equiv d *\left(d t \wedge \frac{\partial A}{\partial t}\right) \equiv \frac{\partial}{\partial t}(d *(d t \wedge A)) \equiv-\frac{\partial}{\partial t}(\check{d} \breve{*} A) \quad \bmod d t,
$$

where we applied the relation $*(d t \wedge \eta)=-\tilde{*} \eta$ which holds for any one-form $\eta$ on $S_{1}^{3}$ without $d t$-part. Thus the function $\check{d}^{*} A:=\check{*} \breve{d} \check{*} A$ does not depend on $t$.

Considering $\check{d}^{*} A$ as a function on $\mathbb{S}^{2}$, we can take a smooth function $\check{\phi}$ on $\mathbb{S}^{2}$ satisfying $\Delta_{\mathbb{S}^{2}} \check{\phi}=-\check{d}^{*} A$ since $\left(\check{d}^{*} A\right) \omega_{\mathbb{S}^{2}}=\check{*}\left(\check{d}^{*} A\right)=\check{d} \check{*} A$ is exact. If we define a smooth function $\phi$ as the pull-back of $\check{\phi}$ by the projection $S_{1}^{3} \cong \mathbb{S}^{2} \times \mathbb{R} \rightarrow \mathbb{S}^{2}$, then we obtain $\check{d} \check{*}(A+d \phi)=0$. Hence $A^{\prime}=A+d \phi$ satisfies the conditions $\left(1^{\circ}\right)$ and $\left(2^{\circ}\right)$.

Now we prove the uniqueness. Suppose that there is a function $\phi$ on $S_{1}^{3}$ such that both $(V, A)$ and $(V, A+d \phi)$ are the monopoles satisfying $\left(1^{\circ}\right)$ and $\left(2^{\circ}\right)$. Then the monopole $(0, d \phi)$ also satisfies $\left(1^{\circ}\right)$ and $\left(2^{\circ}\right)$. By condition $\left(1^{\circ}\right), \phi$ is independent of $t$. Hence $d \phi=\check{d} \phi$. Together with condition $\left(2^{\circ}\right)$, we obtain $d \phi=0$. So the uniqueness follows.

Proposition 4.4. Let $(V, A)$ be a monopole on $S_{1}^{3}$. Suppose that $A$ satisfies $\left(1^{\circ}\right)$ $A\left(\partial_{t}\right)=0$ and $\left(2^{\circ}\right) \check{d} \check{*} A=0$. Then there exists a unique function $f \in C_{*}^{\infty}\left(S_{1}^{3}\right)$ satisfying (i) $V=\partial_{t} f$ and (ii) $A=-\check{*} \breve{d} f$. Moreover $f$ satisfies the equation $L f=0$, where $L$ is the partial differential operator defined in (2.9).

Proof. Let $(V, A)$ be a monopole on $S_{1}^{3}$ satisfying $\left(1^{\circ}\right)$ and $\left(2^{\circ}\right)$. We first claim that there is a smooth function $F$ on $S_{1}^{3}$ such that $A=-\check{*} \breve{d} F$. Such a function 
is obtained, for example, by putting $F(t, y):=\int_{(t, o)}^{(t, y)} \mathscr{*} A$, where $o \in \mathbb{S}^{2}$ is a fixed point and the integral path is taken on the sphere $\{t\} \times \mathbb{S}^{2} \subset S_{1}^{3}$. Since $\mathbb{S}^{2}$ is simply connected, and by the condition $\left(2^{\circ}\right), F(t, y)$ is a well-defined smooth function. By construction, the condition $A=-\check{*} \breve{d} F$ holds.

Next we claim that $\breve{d}\left(V-\partial_{t} F\right)=0$. Actually,

$$
\begin{aligned}
\check{d}\left(V-\partial_{t} F\right) & =d V-V_{t} d t-\check{d} F_{t}, \\
* \check{d}\left(V-\partial_{t} F\right) & =* d V+V_{t} \omega_{\mathbb{S}^{2}}+d t \wedge \check{*} \check{d} F_{t}=\check{d} A+V_{t} \omega_{\mathbb{S}^{2}},
\end{aligned}
$$

and $\check{d} A+V_{t} \omega_{\mathbb{S}^{2}}=0$ by the monopole equation. Hence $\check{d}\left(V-\partial_{t} F\right)=0$ as required, and this means that $G(t):=V(t, y)-\partial_{t} F(t, y)$ does not depend on $y \in \mathbb{S}^{2}$. Thus, if we put $f(t, y)=F(t, y)+\int_{0}^{t} G(t) d t$, conditions (i) and (ii) are satisfied. The uniqueness of $f$ is obvious since conditions (i) and (ii) characterize $f$ up to constant.

The rest of the statement directly follows from the monopole equation. Indeed,

$$
\begin{aligned}
* d V & =*\left(f_{t t} d t+\check{d} f_{t}\right)=-f_{t t} \cosh ^{2} t \omega_{\mathbb{S}^{2}}-d t \wedge \check{*} \check{d} f_{t}, \\
d A & =-d \check{*} \check{d} f=-d t \wedge(\check{*} \check{d} f)_{t}-\check{d} \check{*} \breve{d} f=-d t \wedge \check{*} \check{d} f_{t}-\left(\Delta_{\mathbb{S}^{2}} f\right) \omega_{\mathbb{S}^{2}} ;
\end{aligned}
$$

hence $0=* d V-d A=(L f) \cosh ^{2} t \omega_{\mathbb{S}^{2}}$.

For monopoles that converge at infinity, we obtain the following correspondence.

Theorem 4.5. There is a natural one-to-one correspondence between the following objects:

1. [generating functions] smooth functions $h \in C_{*}^{\infty}\left(\mathbb{S}^{2}\right)$,

2. [monopole potentials] smooth functions $f \in C_{*}^{\infty}\left(S_{1}^{3}\right)$ satisfying $L f=0$ such that $f(t, y) \rightarrow$ $h_{ \pm}(v) \in C^{\infty}\left(\mathbb{S}^{2}\right)$ and $f_{t}, f_{t t} \rightarrow 0$ as $t \rightarrow \pm \infty$ uniformly for $y$,

3. [equivalence classes of monopoles] gauge equivalence classes of monopoles $[(V, A)]$ such that $V(t, y), V_{t}(t, y) \rightarrow$ 0 as $t \rightarrow \pm \infty$ uniformly for $y$.

Proof. By Theorem 3.1 the correspondence $1 \Leftrightarrow 2$ is obtained by putting $f:=R h$ or $h:=h_{+}$. On the other hand, $2 \Rightarrow 3$ is obtained by putting

$$
V=\partial_{t} f \quad \text { and } \quad A=-\check{*} \check{d} f .
$$

Now we show $3 \Rightarrow 2$. For any $[(V, A)]$ we can take an element $(V, A)$ in this class satisfying conditions $\left(1^{\circ}\right)$ and $\left(2^{\circ}\right)$ in Proposition 4.3. Then by Proposition 4.4 we get a unique $f \in C_{*}^{\infty}\left(S_{1}^{3}\right)$ satisfying (4.6) and $L f=0$.

Remark 4.6. In the notation in Theorem 4.5, the evenness $f \in C_{\text {even } *}^{\infty}\left(S_{1}^{3}\right)$ and the oddness $V \in C_{\text {odd }}^{\infty}\left(S_{1}^{3}\right)$ automatically hold by Theorem 3.1 ,

Admissible monopoles. To apply Theorem 4.5 to the study of self-dual metrics, we need to assume additional conditions for $(V, A)$, that is, $V$ is positive and $V$ is written as in (4.4). Now we introduce the following notion.

Definition 4.7. Let $(V, A)$ be a monopole on $S_{1}^{3}$. Then $(V, A)$ is called admissible if and only if the following conditions hold: $\left(1^{\circ}\right) A\left(\partial_{t}\right)=0,\left(2^{\circ}\right) \check{d} \breve{*} A=0$, and $\left(3^{\circ}\right)$ $V>0$ and $V$ satisfies the convergence $V(t, y) \rightarrow 1$ and $V_{t}(t, y) \rightarrow 0$ as $t \rightarrow \pm \infty$ uniformly for $y$. 
The following corollary is obviously deduced from Theorem 4.5 and its proof.

Corollary 4.8. There is a natural one-to-one correspondence between

- smooth functions $h \in C_{*}^{\infty}\left(\mathbb{S}^{2}\right)$ satisfying $\left|\partial_{t} R h(t, y)\right|<1$, and

- admissible monopoles $(V, A)$,

related by $V=1+\partial_{t} R h$ and $A=-\check{*} \check{d}(R h)$.

For condition (4.4), the following hold.

Proposition 4.9. Let $(V, A)$ be an admissible monopole. Then condition (4.4) in Proposition 4.2 is always satisfied. Thus any admissible monopole $(V, A)$ defines a self-dual metric $\bar{g}_{V, A}$ on $\overline{\mathcal{M}}=S^{2} \times S^{2}$ with respect to a suitable orientation.

Proof. Let $(V, A)$ be an admissible monopole. If we put $\tilde{V}:=V-1$, then by Theorem 4.5 there exists a generating function $h \in C_{*}^{\infty}\left(\mathbb{S}^{2}\right)$ such that $\tilde{V}=\partial_{t} R h=$ $-Q \Delta_{\mathbb{S}^{2}} h$. Since $\tilde{V}$ is odd, it is enough to check the case of $t \rightarrow+\infty$. Using the same spherical coordinate $(\theta, \phi)$ as in the proof of Lemma 2.2. we can write

$$
\tilde{V}\left(t_{0}, y\right)=-\frac{1}{2 \pi} \int_{\Omega_{\left(t_{0}, y\right)}} \Delta_{\mathbb{S}^{2}} h \omega_{\mathbb{S}^{2}}=-\frac{1}{2 \pi} \int_{0}^{\alpha}\left[\int_{0}^{2 \pi} \Delta_{\mathbb{S}^{2}} h(u(\theta, \phi)) d \phi\right] \sin \theta d \theta
$$

where $\alpha$ is defined by $\cos \alpha=\tanh t_{0}$. Since the parameter $\theta$ is defined by $\cos \theta=$ $\tanh t, \theta$ depends only on $\kappa:=e^{-2 t}$. So we can put

$$
\mathscr{F}(\kappa, y):=\int_{0}^{2 \pi} \Delta_{\mathbb{S}^{2}} h(u(\theta(\kappa), \phi)) d \phi
$$

Then we obtain

$$
\tilde{V}\left(t_{0}, y\right)=-\frac{1}{\pi} \int_{0}^{q_{0}^{2}} \frac{\mathscr{F}(\kappa, y)}{(1+\kappa)^{2}} d \kappa,
$$

where $q_{0}=e^{-t_{0}}$. Hence $\tilde{V}(t, y)$ is a smooth function depending only on $y$ and $q^{2}=e^{-2 t}$ and satisfies $\lim _{q \rightarrow+\infty} \tilde{V}(t, y)=0$. Therefore $V$ is written as in (4.4).

Later (Corollary 7.5), we will prove the self-duality of the metric $\bar{g}_{V, A}$ on $S^{2} \times S^{2}$ in a different way from Tod's or Kamada's method. (See 13, 27] or the positive definite case [16] for their method.) By our method, we can determine the 'orientation', that is, we fix a certain orientation on $S^{2} \times S^{2}$ and show that $\bar{g}_{V, A}$ is anti-self-dual with respect to this orientation. Moreover, we will see in Corollary 7.10 that this metric $\bar{g}_{V, A}$ is Zollfrei.

Example. Finally in this section, we deal with examples of monopole solutions obtained by Tod [27] and Kamada [14. Let $\left\{Y_{m}^{l}(y)\right\}_{|m| \leq l}$ be the basis of the eigenspace of $\Delta_{\mathbb{S}^{2}}$ with the eigenvalue $-l(l+1)$ (i.e. $Y_{m}^{l}(y) \in C^{\infty}\left(\mathbb{S}^{2}\right)$ can be taken as the spherical harmonics). Introducing the variable $z=\tanh t$, let $P_{l}(z)$ be the Legendre polynomial of degree $l$, and put $Z_{l}(z):=\partial_{z} P_{l}(z)$. In this notation, Tod's monopole solution $(V, A)$ is given by

$$
V=1+\sum_{l \geq 1} \sum_{|m| \leq l} c_{l m} Z_{l}(z) Y_{m}^{l}(y), \quad A=-\sum_{l \geq 1} \sum_{|m| \leq l} c_{l m} P_{l}(z) \check{*} \check{d} Y_{m}^{l}(y),
$$

where $\left\{c_{l m}\right\}$ is a finite collection of real constants with sufficiently small $\left|c_{l m}\right|$. We remark that the above solution $V$ was first obtained by Tod, and later Kamada obtained the above $V$ again with the description of $A$. This monopole solution 
$(V, A)$ is admissible, and the corresponding monopole potential $f \in C_{*}^{\infty}\left(S_{1}^{3}\right)$ and the generating function $h \in C_{*}^{\infty}\left(\mathbb{S}^{2}\right)$ are given by

$$
f=\sum_{l \geq 1} \sum_{|m| \leq l} c_{l m} P_{l}(z) Y_{m}^{l}(y), \quad h=\sum_{l \geq 1} \sum_{|m| \leq l} c_{l m} Y_{m}^{l}(y) .
$$

On the other hand, Kamada constructed another type of monopole solution parametrized by the space of probability measures on the hyperboloids $H_{+}^{3} \sqcup H_{-}^{3}$ in the Minkowski space $\mathbb{R}_{1}^{4}$. However, Theorem 4.5 insists that this type of solution should be gauge equivalent to the above Tod-type admissible monopole at least asymptotically. Actually, Tod's example densely covers the space of admissible monopoles since any generating function $h \in C_{*}^{\infty}\left(\mathbb{S}^{2}\right)$ can be expanded as in the form (4.7).

\section{LOCAL REDUCTION THEORY}

To construct the twistor correspondence for the self-dual metric $\bar{g}_{V, A}$ on $S^{2} \times S^{2}$ obtained from an admissible monopole $(V, A)$, in this section we study the $S^{1}$-bundle $\varpi: M^{4} \rightarrow X^{3}$ and integrable structures on $X$ and $M$.

Einstein-Weyl 3-space. Though we only need the integrable property for the Einstein manifold $\left(S_{1}^{3}, g_{S_{1}^{3}}\right)$, we briefly recall the integrability theorem for general three-dimensional torsion-free Einstein-Weyl structures since there are no differences between the general case and the special case of $S_{1}^{3}$ so far as studying local theory. For the definition of an Einstein-Weyl structure, see [11, 20, 24]. Here we only need the fact that $\left(S_{1}^{3},\left[g_{S_{1}^{3}}\right], \nabla^{S_{1}^{3}}\right)$ is Einstein-Weyl, where $\nabla^{S_{1}^{3}}$ is the LeviCivita connection of the indefinite metric $g_{S_{1}^{3}}$.

Let $X$ be a real 3-manifold and let $\left[g_{X}\right]$ be a conformal structure on $X$ of signature $(-++)$. We fix a metric $g_{X} \in\left[g_{X}\right]$ and a frame $\left\{\underline{E}_{1}, \underline{E}_{2}, \underline{E}_{3}\right\}$ of $T X$ on an open set $\underline{U} \subset X$ so that

$$
g_{X}\left(\underline{E}_{j}, \underline{E}_{k}\right)= \begin{cases}-1 & j=k=1, \\ 1 & j=k=2 \text { or } 3, \\ 0 & \text { otherwise. }\end{cases}
$$

Let $\nabla^{X}$ be a torsion-free connection on $T X$, and $\underline{\omega}$ be its connection form with respect to the above frame. Suppose that $\nabla^{X}$ is compatible with $\left[g_{X}\right]$, that is, $\underline{\omega}$ is written as

$$
\underline{\omega}=\left(\begin{array}{ccc}
\phi & \underline{\omega}_{2}^{1} & \underline{\omega}_{3}^{1} \\
\underline{\omega}_{1}^{2} & \phi & \underline{\omega}_{3}^{2} \\
\underline{\omega}_{1}^{3} & \underline{\omega}_{2}^{3} & \phi
\end{array}\right), \quad\left\{\begin{array}{l}
\underline{\omega}_{1}^{2}=\underline{\omega}_{2}^{1}, \\
\underline{\omega}_{1}^{3}=\underline{\omega}_{3}^{1}, \\
\underline{\omega}_{2}^{3}=-\underline{\omega}_{3}^{2} .
\end{array}\right.
$$

A tangent two-plane $\mathbb{V} \subset T_{x} X(x \in X)$ is called a null plane iff $g_{X}$ degenerates on $\mathbb{V}$, or equivalently, iff $\mathbb{V}$ is tangent to the null cone of $g_{X}$. We put $\mathbb{V}(\zeta):=$ $\operatorname{Span}\left\langle\underline{\mathfrak{m}}_{1}(\zeta), \underline{\mathfrak{m}}_{2}(\zeta)\right\rangle$ for each $\zeta \in \mathbb{R} \cup\{\infty\}=\mathbb{R P}^{1}$, where

$$
\underline{\mathfrak{m}}_{1}(\zeta):=-\underline{E}_{1}+\underline{E}_{2}+\zeta \underline{E}_{3}, \quad \underline{\mathfrak{m}}_{2}(\zeta):=\zeta \underline{E}_{1}+\zeta \underline{E}_{2}-\underline{E}_{3} .
$$

Then $\mathbb{V}(\zeta)$ is a null plane, and any null plane is written in this form.

Now let us define the 'bundle of null planes' on $X$ by

$$
\mathcal{W}_{\mathbb{R}}:=\left\{[a] \in \mathbb{P}\left(T^{*} X\right) \mid g_{X}(a, a)=0\right\} .
$$


Notice that, for each $[a] \in \mathcal{W}_{\mathbb{R}, x}=\mathbb{P}\left(T_{x}^{*} X\right)$, the tangent plane ker $a \subset T_{x} X$ is a null plane. If we define a one-form $\mathfrak{a}(\zeta)$ by

$$
\underline{\mathfrak{a}}(\zeta):=\left(1+\zeta^{2}\right) \underline{E}^{1}+\left(1-\zeta^{2}\right) \underline{E}^{2}+2 \zeta \underline{E}^{3}
$$

using the dual frame $\left\{\underline{E}^{i}\right\}$ of $\left\{\underline{E}_{i}\right\}$, then we obtain $\mathbb{V}(\zeta)=\operatorname{ker} \mathfrak{a}(\zeta)$. Hence the map $\underline{U} \times\left.\mathbb{R P}^{1} \rightarrow \mathcal{W}_{\mathbb{R}}\right|_{U}:(x ; \zeta) \mapsto[\underline{\mathfrak{a}}(\zeta)]_{x}$ gives a local trivialization of $\mathcal{W}_{\mathbb{R}}$. If we introduce coordinates $\theta \in S^{1}$ by $\zeta=\tan \frac{\theta}{2}$ and $\omega=e^{i \theta} \in \mathrm{U}(1)$, then we obtain the trivializations

$$
\underline{U} \times\left.\mathrm{U}(1) \stackrel{\sim}{\longrightarrow} \mathcal{W}_{\mathbb{R}}\right|_{\underline{U}}:(x ; \omega) \longmapsto[\mathfrak{a}]_{x}=\left[2 \omega \underline{E}^{1}+\left(1+\omega^{2}\right) \underline{E}^{2}+i\left(1-\omega^{2}\right) \underline{E^{3}}\right]_{x} .
$$

Let us take an open covering $\left\{\underline{U}_{\alpha}\right\}$ of $X$ and the trivializations of $\mathcal{W}_{\mathbb{R}}$ on each $\underline{U}_{\alpha}$ in the form of (5.5). Then the transition functions are given by the maps $F_{\alpha \beta}: \underline{U}_{\alpha} \cap \underline{U}_{\beta} \rightarrow \operatorname{Aut}(\mathrm{U}(1))$, where $\operatorname{Aut}(\mathrm{U}(1))$ is the Möbius transform on U(1). If $\left(X,\left[g_{X}\right]\right)$ is space-time orientable, these transition functions can be taken so that $F_{\alpha \beta}: \underline{U}_{\alpha} \cap \underline{U}_{\beta} \rightarrow \operatorname{Aut}(\mathbb{D})$, where

$$
\mathbb{D}:=\{\omega \in \mathbb{C}|| \omega \mid \leq 1\}
$$

and $\operatorname{Aut}(\mathbb{D})$ is the holomorphic automorphism on $\mathbb{D}$. Hence if $\left(X,\left[g_{X}\right]\right)$ is spacetime orientable, we can define the $\mathbb{D}$-bundle $\mathcal{W}_{+} \rightarrow X$ associated with the $\mathrm{U}(1)$ bundle $\mathcal{W}_{\mathbb{R}} \rightarrow X$. Notice that we obtain a local trivialization $\underline{U} \times\left.\mathbb{D} \stackrel{\sim}{\longrightarrow} \mathcal{W}_{+}\right|_{U}$ by the same equation as (5.5) considering $\omega \in \mathbb{D}$. We remark that $\mathcal{W}_{+}$is also defined intrinsically as the bundle of complex null planes satisfying an orientation compatibility condition (see [24]). We note that the fiber coordinates $\zeta$ and $\omega$ are related by $\zeta=i \frac{1-\omega}{1+\omega}$, and the disk $\mathbb{D}=\{|\omega| \leq 1\}$ corresponds to the upper half plane $\{\zeta \in \mathbb{C} \mid \operatorname{Im} \zeta \geq 0\}$.

Since the connection $\nabla^{X}$ is compatible with $g_{X}, \mathcal{W}_{\mathbb{R}}$ is equipped with a natural connection which we also denote by $\nabla^{X}$. Let $\tilde{v} \in T_{(x ; \zeta)} \mathcal{W}_{\mathbb{R}}$ be the horizontal lift of a vector $v \in T_{x} X$ with respect to $\nabla^{X}$. Then by a direct calculation, we obtain the following lifting formula:

$$
\tilde{v}=v+\frac{1}{2}\left(\left(1+\zeta^{2}\right) \underline{\omega}_{3}^{2}+\left(1-\zeta^{2}\right) \underline{\omega}_{3}^{1}-2 \zeta \underline{\omega}_{2}^{1}\right)(v) \frac{\partial}{\partial \zeta} .
$$

Let $\underline{\mathfrak{m}}_{j}(j=1,2)$ be the tautological lift of $\underline{\mathfrak{m}}_{j}$ on $\mathcal{W}_{\mathbb{R}}$, i.e. $\left(\underline{\mathfrak{\mathfrak { m }}}_{j}\right)_{(x ; \zeta)}=\left(\underline{\mathfrak{m}}_{j}(\zeta)_{x}\right)$, where $(\cdot)^{\sim}$ is the horizontal lift given by (5.6). We define a two-plane distribution on $\mathcal{W}_{\mathbb{R}}$ by $\underline{\mathcal{D}}:=\operatorname{Span}\left\langle\underline{\mathfrak{\mathfrak { m }}}_{1}, \tilde{\mathfrak{\mathfrak { m }}}_{2}\right\rangle$. The integrability of the Einstein-Weyl condition is stated as follows.

Proposition 5.1. The pair $\left(\left[g_{X}\right], \nabla^{X}\right)$ is Einstein-Weyl iff the two-plane distribution $\underline{\mathcal{D}}$ is Frobenius integrable.

See 24] (Proposition 3.9) for the proof.

Indefinite anti-self-dual 4-space. Next we summarize the integrable property for a 4-dimensional anti-self-dual conformal structure of indefinite signature. Let $M$ be a real 4-manifold and $\left[g_{M}\right]$ be a conformal structure on $M$ of signature $(--++)$. We fix $g_{M} \in\left[g_{M}\right]$ and a frame $\left\{E_{0}, E_{1}, E_{2}, E_{3}\right\}$ of $T M$ on an open set $U \subset M$ so 
that

$$
g_{M}\left(E_{j}, E_{k}\right)= \begin{cases}-1 & j=k=0 \text { or } 1 \\ 1 & j=k=2 \text { or } 3 \\ 0 & \text { otherwise. }\end{cases}
$$

The connection form $\omega$ of the Levi-Civita connection $\nabla$ of $g_{M}$ with respect to the above frame is written as

$$
\omega=\left(\begin{array}{cccc}
0 & \omega_{1}^{0} & \omega_{2}^{0} & \omega_{3}^{0} \\
\omega_{0}^{1} & 0 & \omega_{2}^{1} & \omega_{3}^{1} \\
\omega_{0}^{2} & \omega_{1}^{2} & 0 & \omega_{3}^{2} \\
\omega_{0}^{3} & \omega_{1}^{3} & \omega_{2}^{3} & 0
\end{array}\right), \quad\left\{\begin{array} { l } 
{ \omega _ { 0 } ^ { 1 } = - \omega _ { 1 } ^ { 0 } , } \\
{ \omega _ { 0 } ^ { 2 } = \omega _ { 2 } ^ { 0 } , } \\
{ \omega _ { 0 } ^ { 3 } = \omega _ { 3 } ^ { 0 } , }
\end{array} \quad \left\{\begin{array}{l}
\omega_{1}^{2}=\omega_{2}^{1}, \\
\omega_{1}^{3}=\omega_{3}^{1}, \\
\omega_{2}^{3}=-\omega_{3}^{2} .
\end{array}\right.\right.
$$

We have the eigenspace decomposition $\wedge^{2} T M=\wedge_{+} \oplus \wedge_{-}$with respect to the Hodge operator on $M$, where $\wedge_{ \pm}$is the \pm 1 -eigenspace. Using the above frame $\left\{E_{j}\right\}$, we can write

$$
\wedge_{+}=\operatorname{Span}\left\langle\varphi_{1}, \varphi_{2}, \varphi_{3}\right\rangle, \quad\left\{\begin{array}{l}
\sqrt{2} \varphi_{1}=E_{0} \wedge E_{1}+E_{2} \wedge E_{3}, \\
\sqrt{2} \varphi_{2}=E_{0} \wedge E_{2}+E_{1} \wedge E_{3}, \\
\sqrt{2} \varphi_{3}=E_{0} \wedge E_{3}-E_{1} \wedge E_{2} .
\end{array}\right.
$$

Similarly, we have the decomposition $\wedge^{2} T^{*} M=\wedge^{+} \oplus \wedge^{-}$, and we can take a frame $\left\{\varphi^{1}, \varphi^{2}, \varphi^{3}\right\}$ of $\wedge^{+}$so that $\left\{\varphi_{j}\right\}$ and $\left\{\varphi^{j}\right\}$ are dual to each other.

The Levi-Civita connection $\nabla$ induces a connection on $\wedge_{+}$, which is also denoted by $\nabla$, and its connection form is written as

$$
\eta=\left(\begin{array}{ccc}
0 & \eta_{2}^{1} & \eta_{3}^{1} \\
\eta_{1}^{2} & 0 & \eta_{3}^{2} \\
\eta_{1}^{3} & \eta_{2}^{3} & 0
\end{array}\right), \quad\left\{\begin{array}{r}
\eta_{1}^{2}=\eta_{2}^{1}=\omega_{2}^{1}-\omega_{3}^{0}, \\
\eta_{1}^{3}=\eta_{3}^{1}=\omega_{3}^{1}+\omega_{2}^{0}, \\
-\eta_{2}^{3}=\eta_{3}^{2}=\omega_{3}^{2}-\omega_{1}^{0} .
\end{array}\right.
$$

A tangent two-plane $\mathbb{V} \subset T_{x} M$ is called an $\alpha$-plane iff $g_{M}(\mathbb{V}, \mathbb{V})=\{0\}$ (i.e. $\mathbb{V}$ is contained in the null cone of $\left.g_{M}\right)$ and $\wedge^{2} \mathbb{V} \subset \wedge_{+}$. We put $\mathbb{V}(\zeta)=\operatorname{Span}\left\langle\mathfrak{m}_{1}(\zeta)\right.$, $\left.\mathfrak{m}_{2}(\zeta)\right\rangle$ for each $\zeta \in \mathbb{R} \cup\{\infty\}=\mathbb{R} \mathbb{P}^{1}$, where

$$
\mathfrak{m}_{1}(\zeta):=-\zeta E_{0}-E_{1}+E_{2}+\zeta E_{3}, \quad \mathfrak{m}_{2}(\zeta):=-E_{0}+\zeta E_{1}+\zeta E_{2}-E_{3} .
$$

Then $\mathbb{V}(\zeta)$ is an $\alpha$-plane, and each $\alpha$-plane is written in this form.

We define the 'bundle of $\alpha$-planes' on $M$ by

$$
\mathcal{Z}_{\mathbb{R}}=\left\{[\varphi] \in \mathbb{P}\left(\wedge^{+}\right) \mid g(\varphi, \varphi)=0\right\} .
$$

Notice that for each $[\varphi] \in \mathbb{P}\left(\wedge_{x}^{+}\right)$, the tangent plane $\operatorname{ker} \varphi:=\left\{v \in T_{x} X \mid i(v) \varphi=0\right\}$ is an $\alpha$-plane. If we define $\mathfrak{a}(\zeta) \in \wedge^{+}$by

$$
\mathfrak{a}(\zeta)=-\left(1+\zeta^{2}\right) \varphi^{1}-\left(1-\zeta^{2}\right) \varphi^{2}-2 \zeta \varphi^{3},
$$

then we obtain $\mathbb{V}(\zeta)=\operatorname{ker} \mathfrak{a}(\zeta)$. Hence the map $U \times\left.\mathbb{R P}^{1} \rightarrow \mathcal{Z}_{\mathbb{R}}\right|_{U}:(x ; \zeta) \mapsto[\mathfrak{a}(\zeta)]_{x}$ gives a local trivialization of $\mathcal{Z}_{\mathbb{R}}$. Moreover, if $M$ is space-time orientable, we can define the associated disk bundle $\mathcal{Z}_{+} \rightarrow M$ by a similar method as the case of $\mathcal{W}_{+}$ (see also [19]).

The connection $\nabla$ induces a connection on $\mathcal{Z}_{+}$, which is also denoted by $\nabla$. Let $\tilde{v} \in T_{(x ; \zeta)} \mathcal{Z}_{\mathbb{R}}$ be the horizontal lift of a vector $v \in T_{x} M$ with respect to $\nabla$. Then

$$
\tilde{v}=v+\frac{1}{2}\left(\left(1+\zeta^{2}\right) \eta_{3}^{2}+\left(1-\zeta^{2}\right) \eta_{3}^{1}-2 \zeta \eta_{2}^{1}\right)(v) \frac{\partial}{\partial \zeta} .
$$


Let $\tilde{\mathfrak{m}}_{j}(j=1,2)$ be the tautological lift of $\mathfrak{m}_{j}$ on $\mathcal{Z}_{\mathbb{R}}$, i.e. $\left(\tilde{\mathfrak{m}}_{1}\right)_{(x ; \zeta)}=\left(\mathfrak{m}_{1}(\zeta)_{x}\right)^{r}$, where $(\cdot)^{\sim}$ is the horizontal lift given by (5.12). We define a two-plane distribution on $\mathcal{Z}_{\mathbb{R}}$ by $\mathcal{D}:=\operatorname{Span}\left\langle\tilde{\mathfrak{m}}_{1}, \tilde{\mathfrak{m}}_{2}\right\rangle$. We can extend $\tilde{\mathfrak{m}}_{1}$ and $\tilde{\mathfrak{m}}_{2}$ to complex vector fields on $\mathcal{Z}_{+}$so that they are holomorphic in $\zeta$. We define a complex three-plane distribution $\mathcal{E}$ on $\mathcal{Z}_{+}$by $\mathcal{E}:=\operatorname{Span}\left\langle\tilde{\mathfrak{m}}_{1}, \tilde{\mathfrak{m}}_{2}, \partial_{\bar{\zeta}}\right\rangle$. Then we obtain $\mathcal{E} \cap \overline{\mathcal{E}}=\{0\}$ on $\mathcal{Z}_{+} \backslash \mathcal{Z}_{\mathbb{R}}$; hence $\mathcal{E}$ defines an almost complex structure on $\mathcal{Z}_{+}$so that $\mathcal{E}$ gives the $(0,1)$-vectors.

Proposition 5.2. The following conditions are equivalent:

- the conformal structure $[g]$ is anti-self-dual,

- the two-plane distribution $\mathcal{D}$ on $\mathcal{Z}_{\mathbb{R}}$ is Frobenius integrable,

- the almost complex structure on $\mathcal{Z}_{+} \backslash \mathcal{Z}_{\mathbb{R}}$ defined by $\mathcal{E}$ is integrable.

See [19] (Proposition 3.5 and 7.1) for the proof.

$S^{1}$-fibration. Let $\left(X, g_{X}\right)$ be a pseudo-Riemannian 3-manifold of signature $(-++)$ and apply the above argument for $\left(X,\left[g_{X}\right], \nabla^{X}\right)$, where $\nabla^{X}$ is the LeviCivita connection of $g_{X}$. We put $M:=S^{1} \times X$ and let $\varpi: M \rightarrow X$ be the projection. We fix a solution $(V, A)$ of the monopole equation $* d V=d A$ on $X$, where $V$ is a positive function and $A$ is a one-form on $X$. Then $\Theta=d s+A$ defines a connection on the $S^{1}$-bundle $\varpi: M \rightarrow X$, where $s \in S^{1}$ is the fiber coordinate. We study the following metric on $M$ :

$$
g_{M}:=-V^{-2} \Theta \otimes \Theta+g_{X} .
$$

Notice that $g_{M}$ is conformally equivalent to the metric $g_{V, A}=-V^{-1} \Theta \otimes \Theta+V g_{X}$.

Let us take a local frame $\left\{\underline{E}_{1}, \underline{E}_{2}, \underline{E}_{3}\right\}$ of $T X$ on an open set $\underline{U} \subset X$ so that it satisfies the orthonormal condition (5.1) for $g_{X}$. We write $A=A_{1} \underline{E}^{1}+A_{2} \underline{E}^{2}+$ $A_{3} \underline{E}^{3}$. We define a local frame $\left\{E_{0}, E_{1}, E_{2}, E_{3}\right\}$ of $T M$ on $U:=\varpi^{-1}(\underline{U})$ by

$$
E_{0}=V \frac{\partial}{\partial s}, \quad E_{1}=\underline{E}_{1}-A_{1} \frac{\partial}{\partial s}, \quad E_{2}=\underline{E}_{2}-A_{2} \frac{\partial}{\partial s}, \quad E_{3}=\underline{E}_{3}-A_{3} \frac{\partial}{\partial s} .
$$

Then $\left\{E_{j}\right\}$ satisfies the orthonormal condition (5.7) for $g_{M}$. Notice that the dual frame $\left\{E^{j}\right\}$ of $\left\{E_{j}\right\}$ is given by

$$
E^{0}=V^{-1} \Theta, \quad E^{1}=\varpi^{*} \underline{E}^{1}, \quad E^{2}=\varpi^{*} \underline{E}^{2}, \quad E^{3}=\varpi^{*} \underline{E}^{3} .
$$

Now let us use the same notation as above: $\underline{\omega}, \omega, \underline{\mathfrak{m}}_{j}, \mathfrak{m}_{j}$, and so on.

Lemma 5.3. In the above notation, we obtain the following formulas:

$$
\begin{aligned}
\omega_{1}^{0} & =-\nu_{1} E^{0}+\frac{1}{2} \nu_{3} E^{2}-\frac{1}{2} \nu_{2} E^{3}, & \omega_{2}^{1} & =\varpi^{*} \underline{\omega}_{2}^{1}-\frac{1}{2} \nu_{3} E^{0}, \\
\omega_{2}^{0} & =-\nu_{2} E^{0}-\frac{1}{2} \nu_{3} E^{1}-\frac{1}{2} \nu_{1} E^{3}, & \omega_{3}^{1} & =\varpi^{*} \underline{\omega}_{3}^{1}+\frac{1}{2} \nu_{2} E^{0}, \\
\omega_{3}^{0} & =-\nu_{3} E^{0}+\frac{1}{2} \nu_{2} E^{1}+\frac{1}{2} \nu_{1} E^{2}, & \omega_{3}^{2} & =\varpi^{*} \underline{\omega}_{3}^{2}-\frac{1}{2} \nu_{1} E^{0},
\end{aligned}
$$

where $\nu_{j}:=V^{-1} E_{j} V=V^{-1} \underline{E}_{j} V(j=1,2,3)$.

Proof. By the equation $* d V=d A$, we obtain

$$
\begin{aligned}
d E^{0} & =d\left(V^{-1} \Theta\right)=-V^{-2} d V \wedge \Theta+V^{-1} d A=\left(V^{-1} \Theta\right) \wedge\left(V^{-1} d V\right)+V^{-1} * d V \\
& =E^{0} \wedge\left(\nu_{1} E^{1}+\nu_{2} E^{2}+\nu_{3} E^{3}\right)+\left(-\nu_{1} E^{2} \wedge E^{3}-\nu_{2} E^{1} \wedge E^{3}+\nu_{3} E^{1} \wedge E^{2}\right) \\
& =\sqrt{2}\left(\nu_{1} \varphi^{1}+\nu_{2} \varphi^{2}+\nu_{3} \varphi^{3}\right) .
\end{aligned}
$$

Then the required formulas are deduced by a direct calculation so that $\omega$ satisfies the torsion-free condition $d E^{j}+\sum \omega_{k}^{j} \wedge E^{k}=0$ and the symmetry (5.8). 
Proposition 5.4. In the above notation, we obtain

$$
\tilde{\mathfrak{m}}_{1}=\underline{\tilde{\mathfrak{m}}}_{1}-\left(V \zeta+A\left(\underline{\mathfrak{m}}_{1}\right)\right) \frac{\partial}{\partial s}, \quad \tilde{\mathfrak{m}}_{2}=\underline{\mathfrak{\mathfrak { m }}}_{2}-\left(V+A\left(\underline{\mathfrak{m}}_{2}\right)\right) \frac{\partial}{\partial s} .
$$

Proof. The proof is given by a direct calculation. Here we sketch the proof of the first formula. We have $\mathfrak{m}_{1}=-\zeta E_{0}-E_{1}+E_{2}+\zeta E_{3}=\underline{\mathfrak{m}}_{1}-\zeta E_{0}-A\left(\underline{\mathfrak{m}}_{1}\right) \partial_{s}$ by definition. By the lifting formula (5.12), we obtain

$$
\tilde{\mathfrak{m}}_{1}=\mathfrak{m}_{1}+\frac{1}{2}\left(\left(1+\zeta^{2}\right) \eta_{3}^{2}+\left(1-\zeta^{2}\right) \eta_{3}^{1}-2 \zeta \eta_{2}^{1}\right)\left(\mathfrak{m}_{1}\right) \frac{\partial}{\partial \zeta} .
$$

Evaluating (5.9) and (5.15), and by the lifting formula (5.6), we obtain $\tilde{\mathfrak{m}}_{1}=$ $\underline{\tilde{\mathfrak{m}}}_{1}-\zeta E_{0}-A\left(\underline{\mathfrak{m}}_{1}\right) \partial_{s}=\underline{\tilde{\mathfrak{m}}}_{1}-\left(V \zeta+A\left(\underline{\mathfrak{m}}_{1}\right)\right) \partial_{s}$, as required.

Remark 5.5. By the result of P. E. Jones and K. P. Tod [12, it is natural to expect that, in the above situation, the distribution $\mathcal{D}=\operatorname{Span}\left\langle\tilde{\mathfrak{m}}_{1}, \tilde{\mathfrak{m}}_{2}\right\rangle$ is integrable if and only if $\underline{\mathcal{D}}=\operatorname{Span}\left\langle\underline{\mathfrak{\mathfrak { m }}}_{1}, \underline{\mathfrak{\mathfrak { m }}}_{2}\right\rangle$ is integrable, or equivalently, $g_{M}$ is anti-self-dual if and only if $\left(\left[g_{X}\right], \nabla^{X}\right)$ is Einstein-Weyl. To check this claim directly is, however, very hard. In the special case of $\left(S_{1}^{3}, g_{S_{1}^{3}}\right)$, we prove the integrability of $\mathcal{D}$ by constructing all the integral surfaces of $\mathcal{D}$ (Proposition 7.4).

Finally we see that the projection $\varpi: M \rightarrow X$ induces a map $\Pi:\left(\mathcal{Z}_{+}, \mathcal{Z}_{\mathbb{R}}\right) \rightarrow$ $\left(\mathcal{W}_{+}, \mathcal{W}_{\mathbb{R}}\right)$ if $X$ is space-time orientable (then $M=S^{1} \times X$ is also space-time orientable). For this, notice that $\varpi$ maps each $\alpha$-plane to a null plane since $\varpi_{*}\left(\mathfrak{m}_{j}\right)=\underline{\mathfrak{m}}_{j}$ for $j=1,2$. Recall that $\mathcal{Z}_{\mathbb{R}}$ and $\mathcal{W}_{\mathbb{R}}$ are the spaces of $\alpha$-planes and null planes respectively; hence the natural map $\Pi: \mathcal{Z}_{\mathbb{R}} \rightarrow \mathcal{W}_{\mathbb{R}}$ is induced. By taking local trivializations as above, $\Pi$ is locally described as

$$
\left.\mathcal{Z}_{\mathbb{R}}\right|_{U} \simeq U \times\left.\mathbb{R P}^{1} \longrightarrow \mathcal{W}_{\mathbb{R}}\right|_{\underline{U}} \simeq \underline{U} \times \mathbb{R P}^{1}:(s, x ; \zeta) \longmapsto(x ; \zeta) .
$$

Hence the map $\Pi$ naturally extends to a map $\mathcal{Z}_{+} \rightarrow \mathcal{W}_{+}$. By the formula (5.16), we obtain $\Pi_{*}\left(\tilde{\mathfrak{m}}_{i}\right)=\underline{\mathfrak{m}}_{i}$; hence $\Pi_{*} \mathcal{D}=\underline{\mathcal{D}}$.

\section{STANDARD MODEL}

In this section we study the twistor correspondence for the standard case, that is, the case obtained from the trivial monopole $(V, A)=(1,0)$.

Twistor correspondence for $S_{1}^{3}$. Recall that we identify the de Sitter space $\left(S_{1}^{3}, g_{S_{1}^{3}}\right)$ with the space of oriented small circles on $\mathbb{S}^{2}$. This identification naturally arises from the LeBrun-Mason correspondence for Einstein-Weyl 3-manifolds 20, 24. Here we describe this correspondence.

Let us define submanifolds $\Sigma_{u} \subset S_{1}^{3}$ for each $u \in \mathbb{S}^{2}$ by

$$
\Sigma_{u}:=\left\{(t, y) \in S_{1}^{3} \mid u \in \partial \Omega_{(t, y)}\right\}=\left\{(t, y) \in S_{1}^{3} \mid u \cdot y=\tanh t\right\} .
$$

Then $\Sigma_{u}$ gives a null surface; i.e., $\Sigma_{u}$ is tangent to a null plane at any point on $\Sigma_{u}$. By the correspondence $\Sigma_{u} \leftrightarrow u$, the sphere $\mathbb{S}^{2}$ is identified with the space of these null surfaces on $S_{1}^{3}$.

Let us introduce the affine coordinates $\lambda, \eta \in \mathbb{C P}^{1}$ related with $y, u \in \mathbb{S}^{2}$ by the stereographic projection

$$
\lambda=\frac{y_{2}+i y_{3}}{1+y_{1}}, \quad \eta=\frac{u_{2}+i u_{3}}{1+u_{1}} .
$$


Then the pair $(t, \lambda) \in \mathbb{R} \times \mathbb{C P}^{1}$ can be used as the coordinates on $S_{1}^{3}$. We can check by a direct calculation that

$$
u \cdot y>\tanh t \quad \Longleftrightarrow \quad e^{t}<\left|\frac{\bar{\lambda} \eta+1}{\eta-\lambda}\right|,
$$

and that the null surface (6.1) is written as

$$
\Sigma_{\eta}:=\Sigma_{u}=\left\{(t, \lambda) \in \mathbb{R} \times \mathbb{C P}^{1}\left|e^{t}=\right| \frac{\bar{\lambda} \eta+1}{\eta-\lambda} \mid\right\} .
$$

To adapt the formulation in Section [5, we set the frame $\left\{\underline{E}_{j}\right\}$ of $T S_{1}^{3}$ on the open set $\underline{U}:=\left\{(t, \lambda) \in S_{1}^{3} \mid \lambda \neq \infty\right\}$ by

$$
\underline{E}_{1}=\frac{\partial}{\partial t}, \quad \underline{E}_{2}=\frac{1+|\lambda|^{2}}{2 \cosh t}\left(\frac{\partial}{\partial \lambda}+\frac{\partial}{\partial \bar{\lambda}}\right), \quad \underline{E}_{3}=i \frac{1+|\lambda|^{2}}{2 \cosh t}\left(\frac{\partial}{\partial \lambda}-\frac{\partial}{\partial \bar{\lambda}}\right) .
$$

Notice that $\left\{\underline{E}_{j}\right\}$ satisfies the orthonormal condition (5.1) for the metric $g_{S_{1}^{3}}$. Then the dual frame $\left\{\underline{E}^{j}\right\}$ is given by

$$
\underline{E}^{1}=d t, \quad \underline{E}^{2}=\frac{\cosh t}{1+|\lambda|^{2}}(d \lambda+d \bar{\lambda}), \quad \underline{E}^{3}=-i \frac{\cosh t}{1+|\lambda|^{2}}(d \lambda-d \bar{\lambda}),
$$

and the trivialization (5.4) is written as

$$
\underline{U} \times\left. S^{1} \stackrel{\sim}{\longrightarrow} \mathcal{W}_{\mathbb{R}}\right|_{\underline{U}}:(t, \lambda ; \theta) \longmapsto[\mathfrak{a}]=\left[d t+\frac{\cosh t}{1+|\lambda|^{2}}\left(e^{-i \theta} d \lambda+e^{i \theta} d \bar{\lambda}\right)\right] .
$$

Recall that each point $\left.[\mathfrak{a}] \in \mathcal{W}_{\mathbb{R}}\right|_{x}=\mathbb{P}\left(T_{x}^{*} S_{1}^{3}\right)$ corresponds to the null plane $($ ker $\mathfrak{a}) \subset$ $T_{x} S_{1}^{3}$

Proposition 6.1. For each $[\mathfrak{a}]=(t, \lambda ; \theta) \in \underline{U} \times\left. S^{1} \simeq \mathcal{W}_{\mathbb{R}}\right|_{\underline{U}}$, the corresponding null plane $\mathrm{ker} \mathfrak{a}$ is tangent to the null surface $\bar{\Sigma}_{\eta}$ if and only if

$$
\eta=\frac{-e^{i \theta}+\lambda e^{t}}{\bar{\lambda} e^{i \theta}+e^{t}}
$$

Proof. If we put

$$
F:=|\eta-\lambda|^{2} e^{2 t}-|\bar{\lambda} \eta+1|^{2},
$$

then we can write $\Sigma_{\eta}=\left\{(t, \lambda) \in S_{1}^{3} \mid F=0\right\}$. Suppose $(t, \lambda) \in \Sigma_{\eta}$. Then the tangent plane $T_{(t, \lambda)} \Sigma_{\eta}$ is given by $(\operatorname{ker} d F) \subset T_{(t, \lambda)} S_{1}^{3}$, and by a direct calculation we obtain

$$
d F=2|\bar{\lambda} \eta+1|^{2} \cdot\left[d t-\frac{1+|\eta|^{2}}{(\eta-\lambda)(\lambda \bar{\eta}+1)} d \lambda-\frac{1+|\eta|^{2}}{(\bar{\eta}-\bar{\lambda})(\bar{\lambda} \eta+1)} d \bar{\lambda}\right] .
$$

Comparing with (6.7), we see that the coincidence $($ ker $\mathfrak{a})=($ ker $d F)$ occurs if and only if (6.8) holds.

We put $\mathbb{D}:=\{\omega \in \mathbb{C}|| \omega \mid \leq 1\}$. Since $S_{1}^{3}$ is space-time orientable, we can define the $\mathbb{D}$-bundle $\mathcal{W}_{+}$associated with $\mathcal{W}_{\mathbb{R}}$, and $(t, \lambda ; \omega) \in \underline{U} \times \mathbb{D}$ gives a local coordinate on $\left.\mathcal{W}_{+}\right|_{\underline{U}}$. We define a smooth map $\underline{\mathfrak{f}}: \mathcal{W}_{+} \rightarrow \mathbb{C P}^{1} \times \mathbb{C P}^{1}$ by

$$
\underline{\mathfrak{f}}:(t, \lambda ; \omega) \longmapsto\left(\eta_{1}, \eta_{2}\right)=\left(\frac{-\omega+\lambda e^{t}}{\bar{\lambda} \omega+e^{t}}, \frac{\lambda+e^{t} \omega}{-1+\bar{\lambda} e^{t} \omega}\right)
$$


on $\left.\mathcal{W}_{+}\right|_{\underline{U}}$. Then we obtain the double fibration

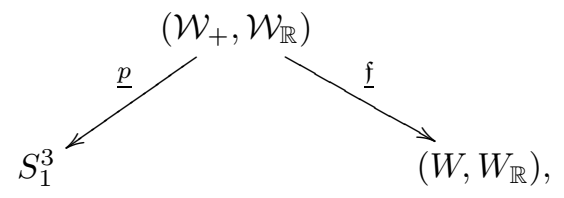

where $\left(W, W_{\mathbb{R}}\right)=\left(\mathbb{C P}^{1} \times \mathbb{C P}^{1}, \mathbb{C P}^{1}\right)$ and $W_{\mathbb{R}} \hookrightarrow W$ is given by $\eta \mapsto\left(\eta, \bar{\eta}^{-1}\right)$. By construction, $\Sigma_{\eta}=p\left(f^{-1}(\eta)\right)$ for each $\eta \in W_{\mathbb{R}} \simeq \mathbb{C P}^{1}$. Notice that if we put $\underline{D}_{(t, \lambda)}:=\underline{f}\left(\underline{p}^{-1}(t, \lambda)\right)$, then $\left\{\underline{D}_{(t, \lambda)}\right\}_{(t, \lambda) \in S_{1}^{3}}$ gives a family of holomorphic disks on $W$ with boundary on $W_{\mathbb{R}}$. Further, by the result in $[20,24]$, the pair $\left(\left[g_{S_{1}^{3}}\right], \nabla^{S_{1}^{3}}\right)$ is the unique torsion-free Einstein-Weyl structure such that $\left\{\Sigma_{\eta}\right\}_{\eta \in W_{\mathbb{R}}}$ gives the family of totally geodesic null surfaces on $S_{1}^{3}$.

As easily seen from (6.3), the domain $\Omega_{(t, \lambda)} \subset \mathbb{S}^{2}$ coincides with the image of the map

$$
\mathbb{D} \longrightarrow \mathbb{C P}^{1}: \omega \longmapsto \eta_{1}(t, \lambda ; \omega)=\frac{-\omega+\lambda e^{t}}{\bar{\lambda} \omega+e^{t}}
$$

under the identification $\mathbb{S}^{2} \stackrel{\sim}{\rightarrow} \mathbb{C P}^{1}$ via stereographic projection. In particular, the oriented small circle $\partial \Omega_{(t, \lambda)}$ coincides with the boundary circle $\partial \underline{D}_{(t, \lambda)} \subset W_{\mathbb{R}}$.

Quaternionic description of $S^{2} \times S^{2}$. Let $\left\{e_{0}, e_{1}, e_{2}, e_{3}\right\}$ be the standard orthonormal basis of the Euclidean space $\mathbb{R}^{4}$ and identify $\mathbb{R}^{4}$ with the quaternion field $\mathbb{H}$ by

$$
a e_{0}+b e_{1}+c e_{2}+d e_{3} \longleftrightarrow a+b i+c j+d k \in \mathbb{H} .
$$

Let $\wedge^{2} \mathbb{R}^{4}=\wedge_{+} \mathbb{R}^{4} \oplus \wedge_{-} \mathbb{R}^{4}$ be the eigenspace decomposition for the Hodge operator on $\mathbb{R}^{4}$. The basis of $\wedge_{ \pm} \mathbb{R}^{4}$ is given by

$$
\begin{aligned}
& \psi_{1}^{ \pm}=\frac{1}{\sqrt{2}}\left(e_{0} \wedge e_{1} \pm e_{2} \wedge e_{3}\right), \\
& \psi_{2}^{ \pm}=\frac{1}{\sqrt{2}}\left(e_{0} \wedge e_{2} \mp e_{1} \wedge e_{3}\right), \\
& \psi_{3}^{ \pm}=\frac{1}{\sqrt{2}}\left(e_{0} \wedge e_{3} \pm e_{1} \wedge e_{2}\right) .
\end{aligned}
$$

Under the identification $\mathbb{H} \simeq \mathbb{R}^{4}$, we obtain for each $q \in \mathbb{R}^{4}$,

$$
\begin{array}{ll}
\sqrt{2} *\left(q \wedge \psi_{1}^{-}\right)=q i, & \sqrt{2} *\left(q \wedge \psi_{1}^{+}\right)=-i q, \\
\sqrt{2} *\left(q \wedge \psi_{2}^{-}\right)=q j, & \sqrt{2} *\left(q \wedge \psi_{2}^{+}\right)=-j q, \\
\sqrt{2} *\left(q \wedge \psi_{3}^{-}\right)=q k, & \sqrt{2} *\left(q \wedge \psi_{3}^{+}\right)=-k q,
\end{array}
$$

where $*: \wedge^{3} \mathbb{R}^{4} \stackrel{\sim}{\rightarrow} \mathbb{R}^{4}$ is the Hodge operator.

We define a bilinear form $h$ on $\wedge^{2} \mathbb{R}^{4}$ so that it satisfies

$$
\xi_{1} \wedge \xi_{2}=h\left(\xi_{1}, \xi_{2}\right) e_{0} \wedge e_{1} \wedge e_{2} \wedge e_{3}
$$

for any $\xi_{1}, \xi_{2} \in \wedge^{2} \mathbb{R}^{4}$. Then the basis $\left\{\psi_{1}^{-}, \psi_{2}^{-}, \psi_{3}^{-}, \psi_{1}^{+}, \psi_{2}^{+}, \psi_{3}^{+}\right\}$gives an orthonormal frame for $h$ of signature $(---+++)$. Let us define

$$
\begin{aligned}
\mathcal{N} & \left.:=\left\{\psi \in \wedge^{2} \mathbb{R}^{4} \mid h(\psi, \psi)=0 \text { (i.e. } \psi \wedge \psi=0\right)\right\}, \\
Q_{\mathbb{R}} & :=\mathcal{N} / \mathbb{R}_{+} \simeq\left\{\sum x^{l} \psi_{l}^{-}+\sum y^{l} \psi_{l}^{+} \mid x, y \in S^{2}\right\},
\end{aligned}
$$


where the positive real numbers $\mathbb{R}_{+}$act on $\mathcal{N}$ by a scalar multiplication. Then $Q_{\mathbb{R}}$ is diffeomorphic to $S^{2} \times S^{2}$ and $h$ induces an indefinite conformal structure on $Q_{\mathbb{R}}$ of signature $(--++)$ which is denoted by $[h]$. If we define

$$
\mathfrak{S}_{q}:=\left\{\psi \in Q_{\mathbb{R}} \mid q \wedge \psi=0\right\}
$$

for each $q \in S^{3} \subset \mathbb{R}^{4}$, then $\mathfrak{S}_{q}$ gives an $\alpha$-surface on $\left(Q_{\mathbb{R}},[h]\right)$ with respect to the natural orientation on $Q_{\mathbb{R}} \simeq S^{2} \times S^{2}$. Since $\mathfrak{S}_{q}=\mathfrak{S}_{-q}$, the $\alpha$-surface $\mathfrak{S}_{q}$ is determined only on $[q] \in \mathbb{R} \mathbb{P}^{3}$, so we also write $\mathfrak{S}_{[q]}=\mathfrak{S}_{q}$.

By the formula (6.12), we can write

$$
\mathfrak{S}_{q}=\{(x, y) \in S(\operatorname{Im} \mathbb{H}) \times S(\operatorname{Im} \mathbb{H}) \mid x=\bar{q} y q\}
$$

under the identification $q \in S^{3} \simeq \operatorname{Sp}(1)$ and $x, y \in S^{2} \simeq S(\operatorname{Im} \mathbb{H})=\{\xi \in \operatorname{Im} \mathbb{H} \mid$ $\xi \bar{\xi}=1\}$. If we put $q=a+b i+c j+d k$, then the transform $\operatorname{Im} \mathbb{H} \rightarrow \operatorname{Im} \mathbb{H}: y \mapsto \bar{q} y q$ is represented by the matrix

$$
\mathscr{A}(\bar{q}):=\left(\begin{array}{ccc}
a^{2}+b^{2}-c^{2}-d^{2} & 2(a d+b c) & -2(a c-b d) \\
-2(a d-b c) & a^{2}-b^{2}+c^{2}-d^{2} & 2(a b+c d) \\
2(a c+b d) & -2(a b-c d) & a^{2}-b^{2}-c^{2}+d^{2}
\end{array}\right)
$$

with respect to the basis $\{i, j, k\} \in \operatorname{Im} \mathbb{H}$. Then we can write $\mathfrak{S}_{q}=\{(x, y) \in$ $\left.S^{2} \times S^{2} \mid x=\mathscr{A}(\bar{q}) y\right\}$. We remark that $\mathscr{A}: \mathrm{Sp}(1) \rightarrow \mathrm{SO}(3)$ gives a natural double cover. By this expression, we see that $\mathfrak{S}_{q}$ is also an $\alpha$-surface for the standard indefinite metric $g_{0}$ on $S^{2} \times S^{2}$, so we obtain $[h]=\left[g_{0}\right]$.

The bundle of $\alpha$-planes $\hat{\mathcal{Z}}_{\mathbb{R}} \rightarrow Q_{\mathbb{R}}$ is naturally given by

$$
\left.\hat{\mathcal{Z}}_{\mathbb{R}}=\left\{(x, y ;[q]) \in Q_{\mathbb{R}} \times \mathbb{R} \mathbb{P}^{3} \mid(x, y) \in \mathfrak{S}_{[q]} \text { (i.e. } x=\mathscr{A}(\bar{q}) y\right)\right\} .
$$

Since $\left(Q_{\mathbb{R}},\left[g_{0}\right]\right)$ is space-time orientable, we can define the disk bundle $\mathcal{Z}_{+}$associated with $\mathcal{Z}_{\mathbb{R}}$. We will see later (Proposition 6.2) that the projection $\hat{\mathfrak{f}}: \hat{\mathcal{Z}}_{\mathbb{R}} \rightarrow \mathbb{R} \mathbb{P}^{3}$ naturally extends to a fiberwise holomorphic map $\hat{\mathfrak{f}}: \hat{\mathcal{Z}}_{+} \rightarrow \mathbb{C P}^{3}$. Then we obtain the following double fibration (see also [19]):

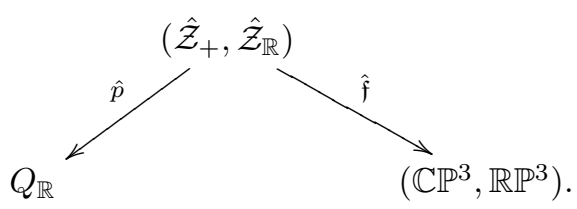

By construction, we have $\hat{p}\left(\hat{\mathfrak{f}}^{-1}([q])\right)=\mathfrak{S}_{[q]}$ for each $[q] \in \mathbb{R P}^{3}$. In this way we obtain the LeBrun-Mason twistor space $\left(\mathbb{C P}^{3}, \mathbb{R P}^{3}\right)$ corresponding to the anti-selfdual 4-manifold $\left(Q_{\mathbb{R}},\left[g_{0}\right]\right)$. Here, the two-plane distribution $\mathcal{D}$ on $\hat{\mathcal{Z}}_{\mathbb{R}}$ is given by the tangent distribution of each fiber of $\hat{\mathfrak{f}}: \hat{\mathcal{Z}}_{\mathbb{R}} \rightarrow \mathbb{R} \mathbb{P}^{3}$.

$S^{1}$-action. Next we study the $S^{1}$-action on $Q_{\mathbb{R}} \simeq S^{2} \times S^{2}$ defined by (4.3). Recall the notation $S_{ \pm}:=\{ \pm \varepsilon\} \times S^{2} \subset S^{2} \times S^{2}, \mathcal{M}:=\left(S^{2} \times S^{2}\right) \backslash\left(S_{+} \sqcup S_{-}\right)$, and so on. We use the coordinate $(s, t, y) \in S^{1} \times \mathbb{R} \times \mathbb{S}^{2}$ on $\mathcal{M}$ as in (4.2). Notice that $\mathcal{M} / S^{1}=\mathbb{R} \times \mathbb{S}^{2} \cong S_{1}^{3}$, and the quotient map $\varpi: \mathcal{M} \rightarrow S_{1}^{3}$ is given by $(s, t, y) \mapsto(t, y)$. As already mentioned, the standard metric $g_{0}$ on $Q_{\mathbb{R}} \simeq S^{2} \times S^{2}$ is conformally equivalent to the metric $g_{1,0}=-d s^{2}+g_{S_{1}^{3}}$ induced from the trivial monopole $(V, A)=(1,0)$.

Now we define the disk bundle $p:\left(\mathcal{Z}_{+}, \mathcal{Z}_{\mathbb{R}}\right) \rightarrow \mathcal{M}$ as the restriction of $\hat{p}$ : $\left(\hat{\mathcal{Z}}_{+}, \hat{\mathcal{Z}}_{\mathbb{R}}\right) \rightarrow Q_{\mathbb{R}}$ on $\mathcal{M}$. By the argument in the previous section, $\varpi: \mathcal{M} \rightarrow S_{1}^{3}$ induces the natural map $\Pi:\left(\mathcal{Z}_{+}, \mathcal{Z}_{\mathbb{R}}\right) \rightarrow\left(\mathcal{W}_{+}, \mathcal{W}_{\mathbb{R}}\right)$. 
Let $\left\{\underline{E}_{1}, \underline{E}_{2}, \underline{E}_{3}\right\}$ be the frame of $T S_{1}^{3}$ on $\underline{U}=\{(t, \lambda) \mid \lambda \neq \infty\}$ defined in (6.5). We introduce a frame $\left\{E_{0}, E_{1}, E_{2}, E_{3}\right\}$ of $T \mathcal{M}$ on $U=\varpi^{-1}(\underline{U})=\{(s, t, \lambda) \mid \lambda \neq \infty\}$ by (5.14), that is,

$$
E_{0}=\frac{\partial}{\partial s}, \quad E_{1}=\underline{E}_{1}, \quad E_{2}=\underline{E}_{2}, \quad E_{3}=\underline{E}_{3} .
$$

If we define $\underline{\mathfrak{m}}_{j}, \mathfrak{m}_{j}$ and so on similarly as in Section 5 , we obtain the trivializations $\underline{U} \times\left.\mathbb{D} \cong \mathcal{W}_{+}\right|_{\underline{U}}$ and $U \times\left.\mathbb{D} \cong \mathcal{Z}_{+}\right|_{U}$. Hence we can use coordinates $(t, \lambda ; \omega)$ on $\left.\mathcal{W}_{+}\right|_{\underline{U}}$ and $(s, \bar{t}, \lambda ; \omega)$ on $\left.\mathcal{Z}_{+}\right|_{U}$. In these coordinates, $\Pi: \mathcal{Z}_{+} \rightarrow \mathcal{W}_{+}$is written as $(s, t, \bar{\lambda} ; \omega) \mapsto(t, \lambda ; \omega)$.

The projection $\varpi$ induces a map between the twistor spaces in the following way. As in (6.16), each $\alpha$-surface $\mathfrak{S}_{q}$ is defined by the equation $x=\mathscr{A}(\bar{q}) y$ for each $q \in S^{3} \subset \mathbb{R}^{4}$. In the coordinate $(s, t, y) \in S^{1} \times \mathbb{R} \times \mathbb{S}^{2} \simeq \mathcal{M}$, this equation is equivalent to the following system:

$$
\begin{aligned}
\frac{e^{i s}}{\cosh t} & =(1, i, 0) \mathscr{A}(\bar{q}) \cdot y, \\
\tanh t & =(0,0,1) \mathscr{A}(\bar{q}) \cdot y .
\end{aligned}
$$

Comparing (6.21) with (6.1), we see that the projection $\varpi$ maps each $\alpha$-surface $\mathfrak{S}_{q}$ to the null surface $\Sigma_{u}$, where $u=(0,0,1) \cdot \mathscr{A}(\bar{q})$. Hence we obtain the natural map

$$
\pi: \mathbb{R P}^{3} \longrightarrow \mathbb{S}^{2} \cong \mathbb{C P}^{1}:[q] \longmapsto u=(0,0,1) \cdot \mathscr{A}(\bar{q})
$$

between the real twistor spaces. We will soon see that $\pi$ extends to the map between complex twistor spaces and obtain the following commutative diagram:

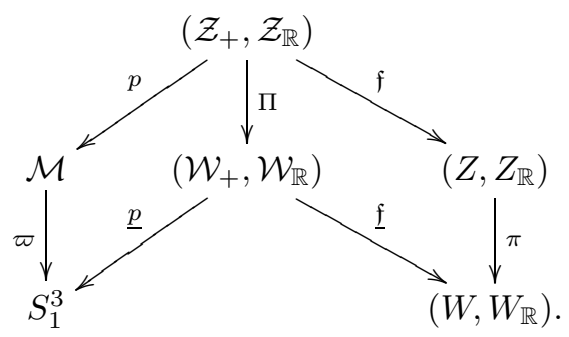

Here $Z_{\mathbb{R}}=\mathbb{R P}^{3} \subset \mathbb{C P}^{3}$ is the standard real submanifold, and $Z \subset \mathbb{C P}^{3}$ is an open set to be defined later.

Explicit description of the double fibration. We set an embedding $\mathbb{H} \cong$ $\mathbb{R}^{4} \rightarrow \mathbb{C}^{4}$ by

$$
\begin{gathered}
q=a+b i+c j+d k \longmapsto\left(z_{0}, z_{1}, z_{2}, z_{3}\right), \\
\sqrt{2} z_{0}=a-i b+c-i d, \quad \sqrt{2} z_{1}=i a-b-i c+d, \\
\sqrt{2} z_{2}=-i a-b+i c+d, \quad \sqrt{2} z_{3}=a+i b+c+i d .
\end{gathered}
$$

Notice that the image of the above embedding is $\left\{z_{3}=\bar{z}_{0}, z_{2}=\bar{z}_{1}\right\}$, and the image of $\operatorname{Sp}(1)=\{q \in \mathbb{H} \mid q \bar{q}=1\}$ is the set of $\left(z_{i}\right)$ satisfying

$$
z_{3}=\bar{z}_{0}, \quad z_{2}=\bar{z}_{1}, \quad\left|z_{0}\right|^{2}+\left|z_{1}\right|^{2}=\left|z_{2}\right|^{2}+\left|z_{3}\right|^{2}=1 .
$$

The above embedding induces the standard embedding $\mathbb{R P}^{3} \rightarrow \mathbb{C P}^{3}$, and we denote its image by $Z_{\mathbb{R}}:=\left\{\left[z_{i}\right] \in \mathbb{C P}^{3} \mid z_{3}=\bar{z}_{0}, z_{2}=\bar{z}_{1}\right\}$. Using this notation, the map $\mathfrak{f}$ is explicitly described in the following way. 
Proposition 6.2. In the above coordinates, the map $\mathfrak{f}: \mathcal{Z}_{\mathbb{R}} \rightarrow Z_{\mathbb{R}}:(s, t, \lambda, \omega) \mapsto\left[z_{i}\right]$ is written as

$$
\begin{aligned}
(s, t, \lambda ; \omega) \longmapsto\left[z_{0}: z_{1}: z_{2}: z_{3}\right] & =\left[e^{i s} \Phi: e^{i s} \Phi \eta: \bar{\eta}: 1\right] \quad\left(\omega=e^{i \theta}\right), \\
\text { where } \eta & =\frac{-\omega+\lambda e^{t}}{\bar{\lambda} \omega+e^{t}}, \quad \Phi=-i \frac{\bar{\lambda} \omega+e^{t}}{\lambda+e^{t} \omega} .
\end{aligned}
$$

Moreover, the extension $\mathfrak{f}: \mathcal{Z}_{+} \rightarrow \mathbb{C P}^{3}$ is written as

$$
(s, t, \lambda ; \omega) \longmapsto\left[z_{0}: z_{1}: z_{2}: z_{3}\right]=\left[e^{i s} \Phi: e^{i s} \Phi \eta_{1}: \eta_{2}^{-1}: 1\right] \quad(|\omega| \leq 1),
$$

where $\eta_{1}$ and $\eta_{2}$ are defined as in (6.9).

We remark that (6.27) can also be written as

$$
(s, t, \lambda ; \omega) \longmapsto\left[-i e^{i s}\left(\bar{\lambda} \omega+e^{t}\right):-i e^{i s}\left(-\omega+\lambda e^{t}\right):-1+\bar{\lambda} e^{t} \omega: \lambda+e^{t} \omega\right] .
$$

We can check by the description (6.27) or (6.28) that the map $f: \mathcal{Z}_{+} \rightarrow \mathbb{C P}^{3}$ naturally extends to a smooth map $\hat{\mathfrak{f}}: \hat{\mathcal{Z}}_{+} \rightarrow \mathbb{C P}^{3}$. (The explicit description of $\hat{\mathfrak{f}}$ near $S_{ \pm}=Q_{\mathbb{R}} \backslash \mathcal{M}$ is given in the proof of Proposition 6.4.)

Proof of 6.2. Recall that the map $\mathfrak{f}: \mathcal{W}_{\mathbb{R}} \rightarrow W_{\mathbb{R}}:(t, \lambda ; \omega) \mapsto \eta$ is written as

$$
\eta=\eta(t, \lambda ; \omega)=\frac{-\omega+\lambda e^{t}}{\bar{\lambda} \omega+e^{t}} \quad\left(\omega=e^{i \theta}\right)
$$

as in Proposition 6.1. Also recall that the map $\pi: \mathbb{R P}^{3} \cong Z_{\mathbb{R}} \rightarrow W_{\mathbb{R}} \cong S^{2}:[q] \mapsto u$ is given by $u=(0,0,1) \mathscr{A}(\bar{q})$. Since $\eta \in \mathbb{C P}^{1}$ and $u \in S^{2}$ are related by stereographic projection, we obtain

$$
\eta=\frac{u_{2}+i u_{3}}{1+u_{1}}=\frac{i a-b-i c+d}{a-i b+c-i d}=\frac{z_{1}}{z_{0}},
$$

where $\left(z_{i}\right) \in \mathbb{C}^{4}$ is the image of $q \in \operatorname{Sp}(1)$. On the other hand, from equation (6.20), we obtain

$$
\frac{e^{i s}}{\cosh t}=i \frac{\left(z_{0}+\bar{\lambda} z_{1}\right)\left(\lambda z_{0}-z_{1}\right)}{2\left(1+|\lambda|^{2}\right)} .
$$

By conditions (6.25) and (6.29), there exists $c \in S^{1}$ satisfying

$$
\left(z_{0}, z_{1}, z_{2}, z_{3}\right)=\frac{2}{\sqrt{1+|\eta|^{2}}}\left(e^{\frac{i c}{2}}, \eta e^{\frac{i c}{2}}, \bar{\eta} e^{-\frac{i c}{2}}, e^{-\frac{i c}{2}}\right) .
$$

Evaluating (6.31) to (6.30), we obtain $e^{i c}=e^{i s} \Phi$. Evaluating this to (6.31) again, we obtain the required description (6.26) of $\mathfrak{f}: \mathcal{Z}_{\mathbb{R}} \rightarrow Z_{\mathbb{R}}$.

The description (6.27) is soon obtained so that the extended map $f: \mathcal{Z}_{+} \rightarrow Z$ is holomorphic in $\omega \in \mathbb{D}$.

We need the following lemma in Section 7

Lemma 6.3. Considering $\Phi=\Phi(t, \lambda ; \omega)$ as a function on $\mathcal{W}_{+}$or on $\mathcal{W}_{\mathbb{R}}$, we obtain $\underline{\mathfrak{\mathfrak { m }}}_{1} \Phi=i \zeta \Phi$ and $\underline{\mathfrak{\mathfrak { m }}}_{2} \Phi=i \Phi$.

Proof. It is enough to check on $\mathcal{W}_{\mathbb{R}}$. Recall that the distribution $\mathcal{D}=\operatorname{Span}\left\langle\tilde{\mathfrak{m}}_{1}, \tilde{\mathfrak{m}}_{2}\right\rangle$ on $\mathcal{Z}_{\mathbb{R}}$ is tangent to each fiber of $\mathfrak{f}: \mathcal{Z}_{\mathbb{R}} \rightarrow Z_{\mathbb{R}}$. Thus we obtain $\tilde{\mathfrak{m}}_{j}\left(e^{i s} \Phi\right)=0$ for $j=1,2$ by the explicit description (6.26) of $\mathfrak{f}$. Then by the formula (5.16), we obtain the required equations since we are now studying the case of the trivial monopole $(V, A)=(1,0)$. 
We remark that, since the distribution $\underline{\mathcal{D}}=\operatorname{Span}\left\langle\underline{\mathfrak{\mathfrak { m }}}_{1}, \tilde{\mathfrak{m}}_{2}\right\rangle$ on $\mathcal{W}_{\mathbb{R}}$ is tangent to each fiber of $\mathfrak{f}: \mathcal{W}_{\mathbb{R}} \rightarrow W_{\mathbb{R}}$, we obtain $\underline{\tilde{\mathfrak{m}}}_{j} \eta=\underline{\mathfrak{\mathfrak { m }}}_{j} \bar{\eta}=0$ for $j=1,2$ on $\mathcal{W}_{\mathbb{R}}$, where $\eta=\eta(t, \lambda ; \omega)$ is defined above. Extending holomorphically, we also obtain $\underline{\mathfrak{m}}_{j} \eta_{k}=0$ for $j=1,2$ and $k=1,2$ on $\mathcal{W}_{+}$.

The twistor space. Now let us define an open set $Z \subset \mathbb{C P}^{3}$ by

$$
Z:=\mathbb{C P}^{3} \backslash\left(L_{+} \sqcup L_{-}\right), \quad \text { where } \quad\left\{\begin{array}{l}
L_{+}=\left\{\left[z_{i}\right] \in \mathbb{C P}^{3} \mid z_{2}=z_{3}=0\right\}, \\
L_{-}=\left\{\left[z_{i}\right] \in \mathbb{C P}^{3} \mid z_{0}=z_{1}=0\right\} .
\end{array}\right.
$$

Further, let us define the holomorphic map $\pi: Z \rightarrow W=\mathbb{C P}^{1} \times \mathbb{C P}^{1}$ by

$$
\pi:\left[z_{0}: z_{1}: z_{2}: z_{3}\right] \longmapsto\left(\eta_{1}, \eta_{2}\right)=\left(\frac{z_{1}}{z_{0}}, \frac{z_{3}}{z_{2}}\right) .
$$

Recall that we defined $Z_{\mathbb{R}}:=\left\{\left[z_{i}\right] \in \mathbb{C P}^{3} \mid z_{3}=\bar{z}_{0}, z_{2}=\bar{z}_{1}\right\}$. Since $\pi\left(Z_{\mathbb{R}}\right)=$ $\left\{\left(\eta_{1}, \eta_{2}\right) \in W \mid \eta_{2}=\bar{\eta}_{1}^{-1}\right\}=W_{\mathbb{R}}$, we obtain the map $\pi:\left(Z, Z_{\mathbb{R}}\right) \rightarrow\left(W, W_{\mathbb{R}}\right)$. Notice that this definition of $\pi$ agrees with the above definition of $\pi: Z_{\mathbb{R}} \rightarrow W_{\mathbb{R}}$ in (6.22) or 6.29).

The set $Z$ is also obtained in the following way. Recall that the $S^{1}$-action on $\mathcal{M}$ is written as $\alpha:(s, t, \lambda) \mapsto(s+\alpha, t, \lambda)$. Then by (6.26), the natural $S^{1}$-action on $Z_{\mathbb{R}}$ is induced and is written as $\alpha:\left[z_{0}: z_{1}: z_{2}: z_{3}\right] \mapsto\left[e^{i \alpha} z_{0}: e^{i \alpha} z_{1}: z_{2}: z_{3}\right]$. This $S^{1}$-action naturally extends to the holomorphic $\mathbb{C}^{*}$-action on $\mathbb{C P}^{3}$ given by

$$
\mu \cdot\left[z_{0}: z_{1}: z_{2}: z_{3}\right] \longmapsto\left[\mu z_{0}: \mu z_{1}: z_{2}: z_{3}\right] \quad\left(\mu \in \mathbb{C}^{*}\right) .
$$

Then $L_{+} \sqcup L_{-}$is just the fixed point set and $Z$ is the free part of this action. Notice that the map $\pi: Z \rightarrow W$ is nothing but the quotient map of the above $\mathbb{C}^{*}$-action.

By the description (6.28), we find that the image of $\mathfrak{f}: \mathcal{Z}_{+} \rightarrow \mathbb{C P}^{3}$ is contained in $Z$. In this way we have obtained the commutative diagram (6.23).

Holomorphic disks. We have already defined the holomorphic disk $\underline{D}_{(t, \lambda)}:=$ $\underline{\mathfrak{f}}\left(\underline{p}^{-1}(t, \lambda)\right)$ for each $(t, \lambda) \in \mathbb{R} \times \mathbb{C P}^{1} \simeq S_{1}^{3}$. Similarly, on the diagram (6.18) we put

$$
D_{\xi}:=\hat{\mathfrak{f}}\left(\hat{p}^{-1}(\xi)\right)
$$

for each $\xi \in Q_{\mathbb{R}}$. Then $\left\{D_{\xi}\right\}_{\xi \in Q_{\mathbb{R}}}$ gives a family of holomorphic disks on $\mathbb{C P}^{3}$ with boundaries on $Z_{\mathbb{R}}$. Recall that we defined $Z:=\mathbb{C P}^{3} \backslash\left(L_{+} \sqcup L_{-}\right)$.

Proposition 6.4. The point $\xi \in Q_{\mathbb{R}}$ is contained in $\mathcal{M}$ if and only if $D_{\xi} \subset Z$. Further, if $\xi \in \mathcal{M}$, then $\pi\left(D_{\xi}\right)=\underline{D}_{\varpi(\xi)}$.

Proof. We change the variable $(s, t, \lambda) \in S^{1} \times \mathbb{R} \times \mathbb{C P}^{1} \cong \mathcal{M}$ to $(\alpha, \lambda) \in \mathbb{C} \times \mathbb{C P}^{1}$ by setting $\alpha=e^{t+i s}$. Then $(\alpha, \lambda)$ gives a coordinate on an open neighborhood $O$ of $S_{-}$, where $S_{ \pm}=\{ \pm \varepsilon\} \times S^{2}$. Notice that $S_{-}=\{(\alpha, \lambda) \in O \mid \alpha=0\}$ and $(\alpha, \lambda)=(0, \lambda)$ corresponds to the point $(-\varepsilon, \lambda)$.

Now recall that $\mathfrak{f}: \mathcal{Z}_{+} \rightarrow Z$ is explicitly written as (6.27). Let us introduce a variable $\omega^{\prime}:=e^{i s} \Phi(t, \lambda ; \omega)$. If $e^{t}<|\lambda|$, then $\omega \mapsto \Phi(t, \lambda ; \omega)$ defines an automorphism on $\mathbb{D}$. Hence we can assume that $\omega^{\prime} \in \mathbb{D}$ on $O$ by shrinking $O$ if needed. Then the triple $\left(\alpha, \lambda ; \omega^{\prime}\right)$ gives a local coordinate on $\left.\hat{\mathcal{Z}}_{+}\right|_{O}$. We obtain that the map $\hat{\mathfrak{f}}:\left.\hat{\mathcal{Z}}_{+}\right|_{O} \rightarrow \mathbb{C P}^{3}$ is written as $\left(\alpha, \lambda ; \omega^{\prime}\right) \longmapsto\left[\omega^{\prime}: \omega^{\prime} \eta_{1}: \eta_{2}^{-1}: 1\right]$, where

$$
\eta_{1}=\frac{\left(1+|\alpha|^{2}\right) \lambda \omega^{\prime}+i\left(1+|\lambda|^{2}\right) \alpha}{\left(|\alpha|^{2}-|\lambda|^{2}\right) \omega^{\prime}}, \quad \eta_{2}=\frac{|\alpha|^{2}-|\lambda|^{2}}{-i\left(1+|\lambda|^{2}\right) \bar{\alpha} \omega^{\prime}+\left(1+|\alpha|^{2}\right) \bar{\lambda}} .
$$


Evaluating $\alpha=0$, we obtain that the disk $D_{(-\varepsilon, \lambda)}$ is given by

$$
\omega^{\prime} \longmapsto\left[\omega^{\prime}:-\omega^{\prime} \bar{\lambda}^{-1}:-\lambda^{-1}: 1\right] \quad\left(\left|\omega^{\prime}\right| \leq 1\right) .
$$

By a similar argument, the disk $D_{(\varepsilon, \lambda)}$ is given by

$$
\omega^{\prime} \longmapsto\left[1: \lambda: \omega^{\prime} \bar{\lambda}: \omega^{\prime}\right] \quad(|\omega| \leq 1) .
$$

Hence each disk $D_{( \pm \varepsilon, \lambda)}$ intersects with $L_{+}$or $L_{-}$, so we obtain $D_{( \pm \varepsilon, \lambda)} \not \subset Z$.

On the other hand, we have $D_{\xi} \subset Z$ for any $\xi \in \mathcal{M}$ since the image of $\mathfrak{f}: \mathcal{Z}_{+} \rightarrow$ $\mathbb{C P}^{3}$ is contained in $Z$. Hence $\xi \in \mathcal{M}$ if and only if $D_{\xi} \subset Z$. The rest of the statement is obvious by the description (6.27).

Compactification of $S_{1}^{3}$. To study the geometry on $S_{1}^{3}$, it is convenient to consider its compactification. Such a picture is actually significant in the study of LeBrun-Mason correspondence for Einstein-Weyl 3-manifolds (see [20, 24]).

Let $\widehat{S}_{1}^{3}:=Q_{\mathbb{R}} / S^{1}$ be the quotient space, and $\hat{\varpi}: Q_{\mathbb{R}} \rightarrow \widehat{S}_{1}^{3}$ be the quotient map. Let us write $\hat{\varpi}( \pm \varepsilon, y)=( \pm \infty, y) \in \widehat{S}_{1}^{3}$. Then $\widehat{S}_{1}^{3} \simeq[-\infty,+\infty] \times S^{2}$ is considered as the natural compactification of $S_{1}^{3} \simeq \mathbb{R} \times S^{2}$, where $[-\infty,+\infty]$ is the natural compactification of $\mathbb{R}$ with two extra points $\pm \infty$.

If we take the limit $t \rightarrow \pm \infty$ for the disks $\underline{D}_{(t, \lambda)}$ on $\left(W, W_{\mathbb{R}}\right)$, then we obtain not a disk but a marked $\mathbb{C P}^{1}$. Actually by (6.9), if we put $\underline{D}_{(+\infty, \lambda)}:=\lim _{t \rightarrow+\infty} \underline{D}_{(t, \lambda)}$, then $\underline{D}_{(+\infty, \lambda)}$ is given by

$$
\{\lambda\} \times \mathbb{C P}^{1} \subset W .
$$

In this limit, the boundaries $\partial \underline{D}_{(t, \lambda)}$ shrink to the point $P_{(+\infty, \lambda)}:=\left(\lambda, \bar{\lambda}^{-1}\right) \in$ $W_{\mathbb{R}}$ which is considered as the marking point of $\underline{D}_{(+\infty, \lambda)}$. Similarly, $\underline{D}_{(-\infty, \lambda)}:=$ $\lim _{t \rightarrow-\infty} \underline{D}_{(t, \lambda)}$ is given by

$$
\mathbb{C P}^{1} \times\{-\lambda\} \subset W
$$

equipped with the marking point at $P_{(-\infty, \lambda)}:=\left(-\bar{\lambda}^{-1},-\lambda\right)$. Notice that by (6.34) or (6.35), we obtain $\pi\left(D_{( \pm \varepsilon, \lambda)} \cap Z\right)=P_{( \pm \infty, \lambda)}$.

Now let us define the maps $\chi_{ \pm}: W_{\mathbb{R}} \rightarrow \partial \widehat{S}_{1}^{3}$ by $\chi_{ \pm}\left(P_{( \pm \infty, \lambda)}\right)=( \pm \infty, \lambda)$. Then we can check that $\widehat{\Sigma}_{\eta}=\Sigma_{\eta} \sqcup\left\{\chi_{+}(\eta), \chi_{-}(\eta)\right\}$ for each $\eta \in W_{\mathbb{R}}$, where $\widehat{\Sigma}_{\eta}$ is the compactification of the null surface $\Sigma_{\eta}$ in $\widehat{S}_{1}^{3}$. Similarly, if we put $\mathfrak{C}_{\left(\eta_{1}, \eta_{2}\right)}:=\underline{p}\left(\mathfrak{f}^{-1}\left(\eta_{1}, \eta_{2}\right)\right)$ for $\left(\eta_{1}, \eta_{2}\right) \in W \backslash W_{\mathbb{R}}$, then we obtain $\widehat{\mathfrak{C}}_{\left(\eta_{1}, \eta_{2}\right)}=\mathfrak{C}_{\left(\eta_{1}, \eta_{2}\right)} \sqcup\left\{\chi_{+}\left(\eta_{1}\right), \chi_{-}\left(\eta_{2}\right)\right\}$, where $\widehat{\mathfrak{C}}_{\left(\eta_{1}, \eta_{2}\right)}$ is the compactification of $\mathfrak{C}_{\left(\eta_{1}, \eta_{2}\right)}$. We remark that $\mathfrak{C}_{\left(\eta_{1}, \eta_{2}\right)} \simeq \mathbb{R}$ is a time-like geodesic on $S_{1}^{3}$ (see [24]).

Finally we remark that, in the picture of the correspondence $S_{1}^{3} \ni(t, y) \leftrightarrow$ $\Omega_{(t, y)} \subset \mathbb{S}^{2}$, the $\operatorname{limit}_{\lim _{t \rightarrow+\infty}} \Omega_{(t, y)}$ shrinks to a point $y \in \mathbb{S}^{2}$ while $\lim _{t \rightarrow-\infty} \Omega_{(t, y)}$ wraps the whole $\mathbb{S}^{2}$ and closes at the point $y \in \mathbb{S}^{2}$.

\section{TWISTOR CORRESPONDENCE}

Main theorem. In Section 6, we put $Z:=\mathbb{C P}^{3} \backslash\left(L_{+} \sqcup L_{-}\right)$and $Z_{\mathbb{R}}:=\left\{\left[z_{0}: z_{1}\right.\right.$ : $\left.\left.z_{2}: z_{3}\right] \in \mathbb{C P}^{3} \mid z_{3}=\bar{z}_{0}, z_{2}=\bar{z}_{1}\right\}$, and showed the correspondence between the map $\pi:\left(Z, Z_{\mathbb{R}}\right) \rightarrow\left(W, W_{\mathbb{R}}\right)$ and the $S^{1}$-bundle $\varpi: \mathcal{M} \rightarrow S_{1}^{3}$ equipped with the standard metrics.

We now define the deformation of the real submanifold $Z_{\mathbb{R}}$ in $Z$ by

$$
P_{h}:=\left\{\left[z_{0}: z_{1}: z_{2}: z_{3}\right] \in Z \mid z_{3}=\bar{z}_{0} e^{-h\left(z_{1} / z_{0}\right)}, z_{2}=\bar{z}_{1} e^{-h\left(z_{1} / z_{0}\right)}\right\},
$$


where $h$ is a smooth function on $\mathbb{C P}^{1} \cong \mathbb{S}^{2}$. Notice that $P_{h}=Z_{\mathbb{R}}$ if $h \equiv 0$, and that $P_{h}$ is invariant under the $\mathrm{U}(1)$-action on $Z$, which is defined as the restriction of the $\mathbb{C}^{*}$-action (6.32). For any real constant $c$, the holomorphic automorphism

$$
\mathbb{C P}^{3} \longrightarrow \mathbb{C P}^{3}:\left[z_{0}: z_{1}: z_{2}: z_{3}\right] \longmapsto\left[z_{0}: z_{1}: z_{2} e^{c}: z_{3} e^{c}\right]
$$

maps $P_{h}$ to $P_{h+c}$, so $P_{h}$ depends on $h$ essentially up to a constant. So we assume $h \in C_{*}^{\infty}\left(\mathbb{S}^{2}\right)$. Then our goal is the following.

Theorem 7.1. Let $(V, A)$ be an admissible monopole, and $h \in C_{*}^{\infty}\left(\mathbb{S}^{2}\right)$ be the corresponding generating function. Then the self-dual metric on $S^{2} \times S^{2}$ induced by $(V, A)$ is Zollfrei, and its LeBrun-Mason twistor space is given by $\left(\mathbb{C P}^{3}, P_{h}\right)$.

Holomorphic disks. To prove Theorem 7.1, we first construct the family of holomorphic disks, and we recover the $S^{1}$-bundle $\varpi: \mathcal{M} \rightarrow S_{1}^{3}$. Recall that for each $(t, \lambda) \in S_{1}^{3}$, the corresponding holomorphic disk on $W$ with boundary on $W_{\mathbb{R}}$ is given by $\underline{D}_{(t, \lambda)}=\mathfrak{f}\left(\underline{p}^{-1}(t, \lambda)\right)$.

Proposition 7.2. There is a unique family of holomorphic disks $\left\{\mathfrak{D}_{(s, t, \lambda)}\right\}$ on $Z$ with boundaries on $P_{h}$ smoothly parametrized by $(s, t, \lambda) \in S^{1} \times \mathbb{R} \times \mathbb{C P}^{1}$ and satisfying the condition: $\mathfrak{D}_{(s, t, \lambda)}$ is mapped biholomorphically onto $\underline{D}_{(t, \lambda)}$ by $\pi$ : $Z \rightarrow W$.

If we put $\mathcal{M}:=S^{1} \times \mathbb{R} \times \mathbb{C P}^{1}$, we obtain the $S^{1}$-bundle $\varpi: \mathcal{M} \rightarrow S_{1}^{3}:(s, t, \lambda) \mapsto$ $(t, \lambda)$ by the above proposition.

Proof of Proposition 7.2. First notice that the boundary $\partial \underline{D}_{(t, \lambda)}$ is given by the image of the map

$$
\underline{\iota}: \partial \mathbb{D} \longrightarrow W_{\mathbb{R}}: \omega \longmapsto \eta=\eta(\omega)=\frac{-\omega+\lambda e^{t}}{\bar{\lambda} \omega+e^{t}} \quad\left(\omega=e^{i \theta} \in \partial \mathbb{D}\right) .
$$

Then any smooth map $\iota: \partial \mathbb{D} \rightarrow P_{h}$ satisfying $\pi \circ \iota=\underline{\iota}$ is written as

$$
\iota: \omega \longmapsto\left[z_{0}: z_{1}: z_{2}: z_{3}\right]=\left[e^{h(\eta(\omega))} K(\omega): e^{h(\eta(\omega))} K(\omega) \eta(\omega): \overline{\eta(\omega)}: 1\right]
$$

using a $\mathrm{U}(1)$-valued smooth function $K$ on $\partial \mathbb{D}$.

Next we deduce the condition for $K$ so that the map $\iota$ extends to a holomorphic map on the disk $\mathbb{D}=\{|\omega| \leq 1\}$. Let us put $H(t, \lambda ; \omega):=h(\eta(\omega))$ and let

$$
H(t, \lambda ; \omega)=\sum_{k=-\infty}^{\infty} H_{k}(t, \lambda) \omega^{k}
$$

be the Fourier expansion. We put

$$
H_{+}(t, \lambda ; \omega):=\sum_{k>0} H_{k}(t, \lambda) \omega^{k}, \quad H_{-}(t, \lambda ; \omega):=\sum_{k<0} H_{k}(t, \lambda) \omega^{k} .
$$

If $\iota$ extends to a holomorphic map on $\mathbb{D}, K$ must be the form

$$
K(\omega)=e^{\left(H_{+}(\omega)-H_{-}(\omega)\right)} \tilde{K}(\omega),
$$

where $\tilde{K}(\omega)$ is a holomorphic function on $\mathbb{D}$ such that $\tilde{K}\left(e^{i \theta}\right) \in \mathrm{U}(1)$. Then $\iota$ is written as

$$
\begin{gathered}
\iota: \omega \longmapsto\left[z_{0}: z_{1}: z_{2}: z_{3}\right]=\left[e^{2 H_{+}+H_{0}} \tilde{K}(\omega): e^{2 H_{+}+H_{0}} \tilde{K}(\omega) \eta_{1}(\omega):\left(\eta_{2}(\omega)\right)^{-1}: 1\right], \\
\text { where } \quad \eta_{1}=\frac{-\omega+\lambda e^{t}}{\bar{\lambda} \omega+e^{t}}, \quad \eta_{2}=\frac{\lambda+e^{t} \omega}{-1+\bar{\lambda} e^{t} \omega} .
\end{gathered}
$$


If the image of $\iota$ is contained in $Z$, then (i) $\tilde{K}(\omega)$ has a unique zero on $\mathbb{D}$ exactly at the pole of $\eta_{1}(\omega)$, and (ii) $\tilde{K}(\omega)$ has a unique pole on $\mathbb{D}$ exactly at the pole of $\eta_{2}(\omega)$. Hence $\tilde{K}(\omega)$ is written as, using a constant $s \in S^{1}$,

$$
\tilde{K}(\omega)=e^{i s} \Phi(\omega), \quad \text { where } \Phi(\omega)=-i \frac{\bar{\lambda} \omega+e^{t}}{\lambda+e^{t} \omega}
$$

Thus $\iota$ is written as

$$
\iota: \omega \longmapsto\left[z_{0}: z_{1}: z_{2}: z_{3}\right]=\left[e^{2 H_{+}+H_{0}+i s} \Phi: e^{2 H_{+}+H_{0}+i s} \Phi \eta_{1}: \eta_{2}^{-1}: 1\right] .
$$

Let us define $\mathfrak{D}_{(s, t, \lambda)}$ to be the holomorphic disk obtained by (7.3). Then the statement follows since $H_{+}, H_{0}$ and $\Phi$ depend smoothly on $(t, \lambda)$, and are independent of $s$.

Recall that the boundary $\partial \underline{D}_{(t, \lambda)} \subset W_{\mathbb{R}} \simeq \mathbb{C P}^{1}$ corresponds to the oriented small circle $\partial \Omega_{(t, y)}$. Hence, in the above proof, the Fourier coefficient $H_{0}(t, \lambda)$ is written as

$$
H_{0}(t, \lambda)=\frac{1}{2 \pi} \int_{0}^{2 \pi} H\left(t, \lambda ; e^{i \theta}\right) d \theta=\frac{1}{2 \pi} \int_{0}^{2 \pi} h\left(\eta\left(e^{i \theta}\right)\right) d \theta=R h(t, \lambda)
$$

using the transform $R$ defined in (2.6). Here we abused the notation as $R h(t, y)=$ $R h(t, \lambda)$.

Non-admissible deformations. Let $\left\{\mathfrak{D}_{\xi}\right\}_{\xi \in \mathcal{M}}$ be the family of holomorphic disks obtained in Proposition 7.2. Let us denote the interior of the disk $\mathfrak{D}_{\xi}$ by $\mathfrak{D}_{\xi}^{\circ}$. We will see later (Proposition 7.7]) that the family $\left\{\mathfrak{D}_{\xi}^{\circ}\right\}_{\xi \in \mathcal{M}}$ foliates $\pi^{-1}\left(W \backslash W_{\mathbb{R}}\right) \subset$ $Z$ if the corresponding monopole $(V, A)$ is admissible, that is, if the generating function $h \in C_{*}^{\infty}\left(\mathbb{S}^{2}\right)$ satisfies $\left|\partial_{t} R h(t, \lambda)\right|<1$.

On the other hand, in the non-admissible case, we obtain the following.

Proposition 7.3. If $\left|\partial_{t} R h(t, \lambda)\right|>1$ for some $(t, \lambda) \in S_{1}^{3}$, then the family $\left\{\mathfrak{D}_{\xi}^{\circ}\right\}_{\xi \in \mathcal{M}}$ does not give a foliation.

Proof. Suppose that there exists a point $\left(t_{0}, \lambda_{0}\right) \in S_{1}^{3}$ such that $\left|\partial_{t} R h\left(t_{0}, \lambda_{0}\right)\right|>1$. Since $R h(t, \lambda)$ is an even function on $S_{1}^{3}$, we can assume $\partial_{t} R h\left(t_{0}, \lambda_{0}\right)+1<0$ by changing $\left(t_{0}, \lambda_{0}\right)$ with $\left(-t_{0},-\lambda_{0}\right)$ if needed.

Now if we evaluate $\omega=0$ to the description (7.3) of the disk $\mathfrak{D}_{(s, t, \lambda)}$, we find that the disk $\mathfrak{D}_{(s, t, \lambda)}$ contains the point $\left[-i e^{H_{0}+t+i s}:-i e^{H_{0}+t+i s} \lambda:-1: \lambda\right] \in Z \backslash P_{h}$. We claim that the map $S^{1} \times \mathbb{R} \rightarrow Z \backslash P_{h}$ given by

$$
(s, t) \longmapsto\left[-i e^{H_{0}\left(t, \lambda_{0}\right)+t+i s}:-i e^{H_{0}\left(t, \lambda_{0}\right)+t+i s} \lambda_{0}:-1: \lambda_{0}\right]
$$

is not injective. If this map is injective, then the function $H_{0}\left(t, \lambda_{0}\right)+t$ must be monotonic in $t \in \mathbb{R}$. We have, however,

$$
\partial_{t} R h\left(t_{0}, \lambda_{0}\right)+1<0 \quad \text { and } \quad \lim _{t \rightarrow \infty}\left(\partial_{t} R h\left(t, \lambda_{0}\right)+1\right)=1>0 ;
$$

hence the function $H_{0}\left(t, \lambda_{0}\right)+t=R h\left(t, \lambda_{0}\right)+t$ is not monotonic. So the map (7.5) is not injective. This means that some members in $\left\{\mathfrak{D}_{\left(s, t, \lambda_{0}\right)}\right\}_{(s, t) \in S^{1} \times \mathbb{R}}$ intersect with each other at their interior points; hence $\left\{\mathfrak{D}_{\xi}^{\circ}\right\}_{\xi \in \mathcal{M}}$ does not give a foliation.

Double fibration. Next we construct the double fibration. Let $(V, A)$ be the monopole corresponding to $h \in C_{*}^{\infty}\left(\mathbb{S}^{2}\right)$, and suppose that $(V, A)$ is admissible. By Proposition 4.9, we obtain an indefinite metric $\bar{g}_{V, A}$ on $\overline{\mathcal{M}}=S^{2} \times S^{2}$. Here we 
show that this metric is anti-self-dual with respect to the natural orientation on $\mathcal{M}=S^{1} \times \mathbb{R} \times \mathbb{C P}^{1}$.

Let $\left(\hat{\mathcal{Z}}_{+}, \hat{\mathcal{Z}}_{\mathbb{R}}\right)$ be the disk bundle on $S^{2} \times S^{2}$ induced from $\bar{g}_{V, A}$ by the method explained in Section 5. Recall that $\hat{\mathcal{Z}}_{\mathbb{R}}$ is equipped with the two-plane distribution $\mathcal{D}$, which is locally written as $\mathcal{D}=\operatorname{Span}\left\langle\tilde{\mathfrak{m}}_{1}, \tilde{\mathfrak{m}}_{2}\right\rangle$, and that $\bar{g}_{V, A}$ is anti-self-dual if and only if $\mathcal{D}$ is integrable.

Let $\left(\mathcal{Z}_{+}, \mathcal{Z}_{\mathbb{R}}\right):=\left(\left.\hat{\mathcal{Z}}_{+}\right|_{\mathcal{M}},\left.\hat{\mathcal{Z}}_{\mathbb{R}}\right|_{\mathcal{M}}\right)$ be the restriction on $\mathcal{M}$. We take a local trivialization of $\left(\mathcal{Z}_{+}, \mathcal{Z}_{\mathbb{R}}\right)$ on the open set $\underline{U}:=\left\{(t, \lambda) \in S_{1}^{3} \mid \lambda \neq \infty\right\}$ in the following way. We fix a frame $\left\{\underline{E}_{j}\right\}_{j=1,2,3}$ of $T S_{1}^{3}$ on $\underline{U}$ in the same way as in (6.5). We define the frame $\left\{E_{j}\right\}_{j=0,1,2,3}$ of $T \mathcal{M}$ on the open set $U:=\varpi^{-1}(\underline{U})=\{(s, t, \lambda) \in \mathcal{M} \mid \lambda \neq \infty\}$ of $\mathcal{M}$ by (5.14) so that we can apply the argument in Section 5 . Then we obtain the trivialization $U \times\left.\mathbb{D} \cong \mathcal{Z}_{+}\right|_{U}$, and we can use $(s, t, \lambda ; \omega) \in U \times \mathbb{D}$ as a local coordinate on $\left.\mathcal{Z}_{+}\right|_{U}$.

Now let $\left\{\mathfrak{D}_{(s, t, \lambda)}\right\}$ be the holomorphic disks obtained in Proposition 7.2, Noticing the explicit description (7.3) of the disk $\mathfrak{D}_{(s, t, \lambda)}$, we define the map $\left(\left.\mathcal{Z}_{+}\right|_{U},\left.\mathcal{Z}_{\mathbb{R}}\right|_{U}\right) \rightarrow$ $\left(Z, P_{h}\right)$ by

$$
(s, t, \lambda ; \omega) \longmapsto\left[z_{0}: z_{1}: z_{2}: z_{3}\right]=\left[e^{2 H_{+}+H_{0}+i s} \Phi: e^{2 H_{+}+H_{0}+i s} \Phi \eta_{1}: \eta_{2}^{-1}: 1\right] .
$$

It is checked that this map uniquely extends to a smooth map $\mathfrak{f}:\left(\mathcal{Z}_{+}, \mathcal{Z}_{\mathbb{R}}\right) \rightarrow$ $\left(Z, P_{h}\right)$. In this way, we obtain a similar diagram to (6.23). By construction, this diagram commutes.

Proposition 7.4. In the above notation, each fiber of the map $\mathfrak{f}: \mathcal{Z}_{\mathbb{R}} \rightarrow P_{h}$ is tangent to the distribution $\left.\mathcal{D}\right|_{\mathcal{Z}_{\mathbb{R}}}$.

Proof. By the explicit description (7.6) of the map $\mathfrak{f}: \mathcal{Z}_{\mathbb{R}} \rightarrow P_{h}$, it is enough to check that the following formulas hold for $j=1,2$ :

$$
\begin{gathered}
\tilde{\mathfrak{m}}_{j}\left(e^{2 H_{+}+H_{0}+i s} \Phi\right)=0, \\
\tilde{\mathfrak{m}}_{j} \eta_{1}=\tilde{\mathfrak{m}}_{j} \eta_{2}=0,
\end{gathered}
$$

on $\left.\mathcal{Z}_{\mathbb{R}}\right|_{U}$. The equation (7.8) is, however, obvious since the vectors $\Pi_{*}\left(\mathfrak{m}_{j}\right)=\underline{\mathfrak{m}}_{j}$ $(j=1,2)$ and the functions $\eta_{k}(k=1,2)$ are not deformed from the standard case, so $\tilde{\mathfrak{m}}_{j} \eta_{k}=\tilde{\mathfrak{\mathfrak { m }}}_{j} \eta_{k}=0$ for each $j, k$. On the other hand, by Proposition [5.4 the equations (7.7) are equivalent to the following equations:

$$
\left\{\begin{array}{l}
\tilde{\tilde{\mathfrak{m}}}_{1}\left(e^{2 H_{+}+H_{0}} \Phi\right)=i\left(V \zeta+A\left(\underline{\mathfrak{m}}_{1}\right)\right) \cdot e^{2 H_{+}+H_{0}} \Phi, \\
\tilde{\mathfrak{m}}_{2}\left(e^{2 H_{+}+H_{0}} \Phi\right)=i\left(V+A\left(\underline{\mathfrak{m}}_{2}\right)\right) \cdot e^{2 H_{+}+H_{0}} \Phi,
\end{array}\right.
$$

where $\zeta=i \frac{1-\omega}{1+\omega}$. If we apply Lemma 6.3, the wanted equation (7.7) is equivalent to

$$
\left\{\begin{array}{l}
\tilde{\mathfrak{\mathfrak { m }}}_{1}\left(2 H_{+}+H_{0}\right)=i\left((V-1) \zeta+A\left(\underline{\mathfrak{m}}_{1}\right)\right), \\
\tilde{\mathfrak{m}}_{2}\left(2 H_{+}+H_{0}\right)=i\left((V-1)+A\left(\underline{\mathfrak{m}}_{2}\right)\right) .
\end{array}\right.
$$

Now notice that for $|\omega|=1$ we have

$$
\underline{\mathfrak{m}}_{j} H(t, \lambda ; \omega)=\underline{\mathfrak{m}}_{j} h(\eta(\omega))=\frac{\partial h}{\partial \eta} \cdot \underline{\mathfrak{m}}_{j} \eta_{1}+\frac{\partial h}{\partial \bar{\eta}} \cdot \underline{\mathfrak{m}}_{j} \eta_{2}=0
$$

If we use the formula (B.4) in Appendix B, we obtain the following equations:

$$
l_{j} H_{k}+\bar{l}_{j} H_{k-1}+k \delta_{j} H_{k}-(k-1) \bar{\delta}_{j} H_{k-1}=0 \quad(k \in \mathbb{Z}),
$$


where $H(t, \lambda ; \omega)=\sum_{k} H_{k}(t, \lambda) \omega^{k}$. Thus we obtain for $j=1$,

$$
\begin{aligned}
(1+\omega) \tilde{\tilde{\mathfrak{m}}}_{1}\left(2 H_{+}+H_{0}\right) & =2\left(l_{1} H_{1}+\delta_{1} H_{1}\right) \omega+\left(l_{1} H_{0}+\omega \bar{l}_{1} H_{0}\right)=l_{1} H_{0}-\omega \bar{l}_{1} H_{0} \\
& =-(1-\omega) \underline{E}_{1} H_{0}+(1-\omega) \underline{E}_{2} H_{0}+i(1+\omega) \underline{E}_{3} H_{0} .
\end{aligned}
$$

On the other hand, we have $H_{0}(t, \lambda)=R h(t, \lambda)$ and by the hypothesis,

$$
\begin{aligned}
& V=1+\partial_{t} R h=1+\underline{E}_{1} H_{0}, \\
& A=-\check{*} \check{d} R h=\left(\underline{E}_{3} H_{0}\right) \underline{E}^{2}-\left(\underline{E}_{2} H_{0}\right) \underline{E}^{3} .
\end{aligned}
$$

Hence

$$
\begin{array}{r}
-(1-\omega)(V-1)+i A\left((1+\omega) \underline{\mathfrak{m}}_{1}\right)=-(1-\omega) \underline{E}_{1} H_{0}+i A\left(l_{1}+\omega \bar{l}_{1}\right) \\
=-(1-\omega) \underline{E}_{1} H_{0}+(1-\omega) \underline{E}_{2} H_{0}+i(1+\omega) \underline{E}_{3} H_{0} .
\end{array}
$$

By (7.12) and (7.13), we obtain

$$
(1+\omega) \underline{\mathfrak{m}}_{1}\left(2 H_{+}+H_{0}\right)=-(1-\omega)(V-1)+i A\left((1+\omega) \underline{\mathfrak{m}}_{1}\right),
$$

which is equivalent to the first equation of (7.9). The second equation of (7.9) is proved in a similar way.

Corollary 7.5. Let $(V, A)$ be an admissible monopole. Then the metric $\bar{g}_{V, A}$ on $S^{2} \times S^{2}$ induced from $(V, A)$ is anti-self-dual with respect to the natural orientation on $\mathcal{M}=S^{1} \times \mathbb{R} \times \mathbb{C P}^{1}$.

Proof. Notice that the map $\mathfrak{f}: \mathcal{Z}_{\mathbb{R}} \rightarrow P_{h}$ is surjective by construction. Hence each fiber of $\mathfrak{f}: \mathcal{Z}_{\mathbb{R}} \rightarrow P_{h}$ is two-dimensional and is an integral surface of $\mathcal{D}$ by Proposition 7.4. Thus $\mathcal{D}$ is Frobenius integrable. Hence $\bar{g}_{V, A}$ is anti-self-dual on $\mathcal{M}$ by Proposition 5.2. Since $\mathcal{M}$ is dense in $S^{2} \times S^{2}, g$ is anti-self-dual on the whole of $S^{2} \times S^{2}$.

By Proposition [5.2, the complex three-plane distribution $\mathcal{E}=\operatorname{Span}\left\langle\tilde{\mathfrak{m}}_{1}, \tilde{\mathfrak{m}}_{2}, \partial_{\bar{\omega}}\right\rangle$ defines the complex structure on $\mathcal{Z}_{+} \backslash \mathcal{Z}_{\mathbb{R}}$. Since $e^{2 H_{+}+H_{0}+i s} \Phi$ is holomorphic in $\omega \in \mathbb{D}$, the equations (7.7) and (7.8) hold on $\mathcal{Z}_{+}$. Hence the map $\mathfrak{f}: \mathcal{Z}_{+} \rightarrow Z$ is holomorphic on $\mathcal{Z}_{+} \backslash \mathcal{Z}_{\mathbb{R}}$. In this way, we have obtained the following result.

Proposition 7.6. In the above notation, $\mathfrak{f}:\left(\mathcal{Z}_{+} \backslash \mathcal{Z}_{\mathbb{R}}\right) \rightarrow\left(Z \backslash P_{h}\right)$ is holomorphic.

Compactification. Recall that the compactification $\mathcal{M} \hookrightarrow S^{2} \times S^{2}$ is given by $(s, t, \lambda) \mapsto(x, y)$, where $y \stackrel{\sim}{\mapsto} \lambda$ is the stereographic projection and $(s, t) \mapsto x$ is given by (4.2). We have $\left(S^{2} \times S^{2}\right) \backslash \mathcal{M}=S_{+} \sqcup S_{-}$, where $S_{ \pm}=\{ \pm \varepsilon\} \times S^{2}$. Similar to the proof of Proposition 6.4 let us introduce the variables $\alpha=e^{t+i s}$ and $\omega^{\prime}=e^{i s} \Phi(t, \lambda, \omega)$. Then $(\alpha, \lambda)$ gives a coordinate on the small open neighborhood $O \subset S^{2} \times S^{2}$ of $S_{-}$and $\left(\alpha, \lambda ; \omega^{\prime}\right)$ gives a local coordinate on $\left.\hat{\mathcal{Z}}_{+}\right|_{O}$. The map $\mathfrak{f}: \mathcal{Z}_{+} \rightarrow Z$ defined in (7.6) is written as

$$
\mathfrak{f}:\left(\alpha, \lambda ; \omega^{\prime}\right) \longmapsto\left[e^{2 H_{+}+H_{0}} \omega^{\prime}: e^{2 H_{+}+H_{0}} \omega^{\prime} \eta_{1}: \eta_{2}^{-1}: 1\right],
$$

where $\eta_{1}$ and $\eta_{2}$ are given by (6.33). Since the function $H\left(t, \lambda ; e^{i \theta}\right)=h\left(\eta_{1}\left(t, \lambda ; e^{i \theta}\right)\right)$ extends to a smooth function on $\left.\left(\alpha, \lambda ; \omega^{\prime}\right) \in \hat{\mathcal{Z}}_{\mathbb{R}}\right|_{O}$, its Fourier coefficient $H_{k}$ extends to a smooth function on $(\alpha, \lambda) \in O$ for each $k \in \mathbb{Z}$. Hence (17.14) extends to the 
smooth map $\hat{\mathfrak{f}}:\left.\hat{\mathcal{Z}}_{+}\right|_{O} \rightarrow \mathbb{C P}^{3}$. By a similar argument for $S_{+}$, we obtain the smooth map $\hat{\mathfrak{f}}: \hat{\mathcal{Z}}_{+} \rightarrow \mathbb{C P}^{3}$ as an extension of $\mathfrak{f}$. So we get the double fibration

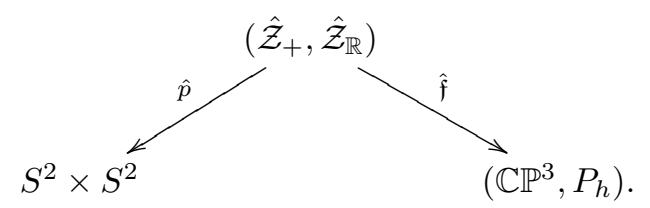

Let us define the holomorphic disks $\left\{\mathfrak{D}_{\xi}\right\}_{\xi \in S^{2} \times S^{2}}$ by $\mathfrak{D}_{\xi}:=\hat{\mathfrak{f}}\left(\hat{p}^{-1}(\xi)\right)$. Of course, this notation agrees with the previous notation of $\mathfrak{D}_{\xi}$ for $\xi \in \mathcal{M}$. Since we have

$$
\begin{gathered}
\left.H_{+}\left(\alpha, \lambda ; \omega^{\prime}\right)\right|_{\alpha=0}=\lim _{t \rightarrow-\infty} H_{+}(t, \lambda, \omega)=0, \\
\left.H_{0}\left(\alpha, \lambda ; \omega^{\prime}\right)\right|_{\alpha=0}=\lim _{t \rightarrow-\infty} H_{0}(t, \lambda, \omega)=h\left(-\bar{\lambda}^{-1}\right),
\end{gathered}
$$

the disk $\mathfrak{D}_{(-\varepsilon, y)}$ is given by the map

$$
\omega^{\prime} \longmapsto\left[e^{h\left(-\bar{\lambda}^{-1}\right)} \omega^{\prime}:-e^{h\left(-\bar{\lambda}^{-1}\right)} \omega^{\prime} \bar{\lambda}^{-1}:-\lambda: 1\right] .
$$

Similarly, we can check that $\mathfrak{D}_{(\varepsilon, y)}$ is given by the map

$$
\omega^{\prime} \longmapsto\left[e^{h(\lambda)}: e^{h(\lambda)} \lambda: \omega^{\prime} \bar{\lambda}: \omega^{\prime}\right] .
$$

The family of holomorphic disks $\left\{\mathfrak{D}_{\xi}\right\}$ on $\left(\mathbb{C P}^{3}, P_{h}\right)$ has the following properties.

Proposition 7.7. The family $\left\{\mathfrak{D}_{\xi}\right\}_{\xi \in S^{2} \times S^{2}}$ satisfies the following conditions:

(1) $\xi \in \mathcal{M}$ if and only if $\mathfrak{D}_{\xi} \subset Z$,

(2) for each disk $\mathfrak{D}_{\xi}$, the class $\left[\mathfrak{D}_{\xi}\right] \in H_{2}\left(\mathbb{C P}^{3}, P ; \mathbb{Z}\right)$ gives a generator, and

(3) $\left\{\mathfrak{D}_{\xi}^{\circ}\right\}_{\xi \in S^{2} \times S^{2}}$ foliates $\mathbb{C P}^{3} \backslash P_{h}$, where $\mathfrak{D}_{\xi}^{\circ}$ is the interior of $\mathfrak{D}_{\xi}$.

Proof. Statement (1) is easily deduced by the above descriptions of $\mathfrak{D}_{\xi}$. To check (2), it is enough to check the case $\xi=( \pm \varepsilon, y) \in S_{ \pm}$since all the disks of $\left\{\mathfrak{D}_{\xi}\right\}$ are homotopic in $\left(\mathbb{C P}^{3}, P_{h}\right)$ to each other, and this is obvious by the above descriptions of $\mathfrak{D}_{( \pm \varepsilon, y)}$.

To prove (3), we show

$\left(1^{\circ}\right)$ the family $\left\{\mathfrak{D}_{\xi}^{\circ}\right\}_{\xi \in \mathcal{M}}$ foliates $Z^{\circ}:=\pi^{-1}\left(W \backslash W_{\mathbb{R}}\right)$, and

$\left(2^{\circ}\right)$ the family $\left\{\mathfrak{D}_{\xi}^{\circ}\right\}_{\xi \in\left(S_{+} \sqcup S_{-}\right)}$foliates $\left(\mathbb{C P}^{3} \backslash Z^{\circ}\right) \backslash P_{h}$.

Here $\left(2^{\circ}\right)$ is obviously deduced by the descriptions of $\mathfrak{D}_{( \pm \varepsilon, y)}$ since

$$
\left(\mathbb{C P}^{3} \backslash Z^{\circ}\right) \backslash P_{h}=\left\{\left[c e^{h(\eta)}: c e^{h(\eta)} \eta: \bar{\eta}: 1\right] \in \mathbb{C P}^{3}\left|\eta, c \in \mathbb{C P}^{1},\right| c \mid \neq 1\right\} .
$$

To check $\left(1^{\circ}\right)$, it is enough to show that $\mathfrak{f}:\left(\mathcal{Z}_{+} \backslash \mathcal{Z}_{\mathbb{R}}\right) \rightarrow Z^{\circ}$ is bijective. For this, we only need to show that the restriction $\mathfrak{f}^{-1}\left(\pi^{-1}\left(\eta_{1}, \eta_{2}\right)\right) \stackrel{\mathfrak{f}}{\rightarrow} \pi^{-1}\left(\eta_{1}, \eta_{2}\right)$ is bijective for each point $\left(\eta_{1}, \eta_{2}\right) \in W \backslash W_{\mathbb{R}}$. We put $\mathcal{C}:=\mathfrak{f}^{-1}\left(\pi^{-1}\left(\eta_{1}, \eta_{2}\right)\right) \subset \mathcal{Z}_{+}$ and $\underline{\mathcal{C}}:=\mathfrak{f}^{-1}\left(\eta_{1}, \eta_{2}\right) \subset \mathcal{W}_{+}$. The set $\underline{\mathcal{C}}$ is diffeomorphic to $\mathbb{R}$. (In fact this is a canonical lift of the time-like geodesic $\mathfrak{C}_{\left(\eta_{1}, \eta_{2}\right)}$. See [24.) The map $\left.\Pi\right|_{\mathcal{C}}: \mathcal{C} \rightarrow \underline{\mathcal{C}}$ is an $S^{1}$-bundle with fiber coordinate $s \in S^{1}$. Notice that $\mathcal{C}$ is a complex submanifold of $\mathcal{Z}_{+} \backslash \mathcal{Z}_{\mathbb{R}}$ since $\mathfrak{f}$ and $\pi$ are holomorphic.

Now we suppose $\eta_{1} \neq \infty$ and $\eta_{2} \neq 0$. First we check that $\left.\mathfrak{f}\right|_{\mathcal{C}}: \mathcal{C} \rightarrow \pi^{-1}\left(\eta_{1}, \eta_{2}\right)$ is injective. In this case we can take a coordinate $\mathbb{C}^{*} \cong \pi^{-1}\left(\eta_{1}, \eta_{2}\right)$ by $\mu \mapsto\left[\mu: \mu \eta_{1}\right.$ : $\left.\eta_{2}^{-1}: 1\right]$. Then $\left.\mathfrak{f}\right|_{\mathcal{C}}$ is written as $\mathcal{F}:(s, t, \lambda ; \omega) \mapsto e^{2 H_{+}+H_{0}+i s} \Phi$ by (7.6). Notice that $\partial_{s} \mathcal{F}=i \mathcal{F}$. If we denote the complex structure on $\mathcal{C}$ by $J$, then $\left(\partial_{s}+i J\left(\partial_{s}\right)\right) \mathcal{F}=0$ 
since $\left(\partial_{s}+i J\left(\partial_{s}\right)\right)$ is a $(0,1)$-vector field. Hence $J\left(\partial_{s}\right) \mathcal{F}=-\mathcal{F}$. Here by $\eta_{1} \neq \infty$ and $\eta_{2} \neq 0$, we obtain $\mathcal{F} \neq 0, \infty$. So $J\left(\partial_{s}\right) \mathcal{F}=-\mathcal{F}$ means that any two fiber-circles of $\mathcal{C} \rightarrow \underline{\mathcal{C}}$ are mapped by $\mathfrak{f}$ to different $\mathrm{U}(1)$-orbits in $\pi^{-1}\left(\eta_{1}, \eta_{2}\right) \cong \mathbb{C}^{*}$. Hence $\mathcal{F}$ is injective.

Next we check the surjectivity of $\mathcal{F}$. For this, it is enough to show that $\lim _{t \rightarrow-\infty} \mathcal{F}$ $=0$ and $\lim _{t \rightarrow+\infty} \mathcal{F}=\infty$. As explained in the last part of Section $6, \mathfrak{C}_{\left(\eta_{1}, \eta_{2}\right)}=$ $\underline{p}\left(\mathfrak{f}^{-1}\left(\eta_{1}, \eta_{2}\right)\right)$ is a time-like geodesic connecting $\chi_{+}\left(\eta_{1}\right)$ and $\chi_{-}\left(\eta_{2}\right)$ in the compactification $\widehat{S}_{1}^{3}$ of $S_{1}^{3}$. Recall that $\varpi: \mathcal{M} \rightarrow S_{1}^{3}$ is naturally compactified to the quotient map $\hat{\varpi}: S^{2} \times S^{2} \rightarrow \widehat{S}_{1}^{3}$. So the set $\varpi^{-1}\left(\mathfrak{C}_{\left(\eta_{1}, \eta_{2}\right)}\right) \simeq \mathbb{R} \times S^{1}$ is compactified to $\hat{\varpi}^{-1}\left(\widehat{\mathfrak{C}}_{\left(\eta_{1}, \eta_{2}\right)}\right) \simeq S^{2}$ in $S^{2} \times S^{2}$ with two extra points $\left(+\varepsilon, \eta_{1}\right)$ and $\left(-\varepsilon, \eta_{2}\right)$. Notice that $\mathcal{C}$ is homeomorphically mapped onto $\varpi^{-1}\left(\mathfrak{C}_{\left(\eta_{1}, \eta_{2}\right)}\right)$ by $p$. For any path $\gamma(\tau)$ in $\mathcal{C}$ with parameter $\tau \in[0, \infty)$ such that $\lim _{\tau \rightarrow \infty} p(\gamma(\tau))=\left(+\varepsilon, \eta_{1}\right)$, we obtain that $\lim _{\tau \rightarrow \infty} \mathfrak{f}(\gamma(\tau))$ is, if it exists, contained in the disk $\mathfrak{D}_{\left(+\varepsilon, \eta_{1}\right)}$. On the other hand, $\lim _{\tau \rightarrow \infty} \mathfrak{f}(\gamma(\tau))$ is, if it exists, contained in the closure $\overline{\pi^{-1}\left(\eta_{1}, \eta_{2}\right)}$. Since the inter-

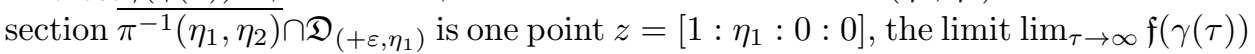
actually exists independently with the path $\gamma(\tau)$ and the limit is above $z$. Hence we obtain $\lim _{t \rightarrow \infty} \mathcal{F}=\infty$. Similarly we can check that $\lim _{t \rightarrow-\infty} \mathcal{F}=0$.

In this way, we have proved that $\left.\mathfrak{f}\right|_{\mathcal{C}}$ is bijective if $\eta_{1} \neq \infty$ and $\eta_{2} \neq 0$. In the case $\eta_{1}=\infty$ or $\eta_{2}=0$, we can check the bijectivity of $\left.\mathfrak{f}\right|_{\mathcal{C}}$ similarly by taking a suitable coordinate $\mathbb{C}^{*} \cong \pi^{-1}\left(\eta_{1}, \eta_{2}\right)$. Thus statement (3) is proved.

Zollfrei condition. On the double fibration (7.15), we set

$$
\mathfrak{S}_{z}:=\hat{p}\left(\hat{\mathfrak{f}}^{-1}(z)\right)=\left\{\xi \in S^{2} \times S^{2} \mid z \in \mathfrak{D}_{\xi}\right\}
$$

for each point $z \in P_{h}$.

Proposition 7.8. The set $\mathfrak{S}_{z}$ is a smoothly embedded $S^{2} \subset S^{2} \times S^{2}$ for each point $z \in P_{h}$.

Proof. We consider the null surface $\Sigma_{\pi(z)}:=\underline{p}\left(\mathfrak{f}^{-1}(\pi(z))\right)$ on $S_{1}^{3}$. By definition, for each $(t, \lambda) \in \Sigma_{\pi(z)}$ we have $\pi(z) \in \partial \underline{D}_{(t, \lambda)}$. Recall that $\left\{\mathfrak{D}_{(s, t, \lambda)}\right\}_{s \in S^{1}}$ gives the family of disks satisfying $\pi\left(\mathfrak{D}_{(s, t, \lambda)}\right)=\underline{D}_{(t, \lambda)}$. Notice that for each $(t, \lambda) \in \Sigma_{\pi(z)}$ there is a unique $s=s(t, \lambda) \in S^{1}$ such that $z \in \partial \mathfrak{D}_{(s, t, \lambda)}$. Actually if we write $z=\left[c e^{h(\eta)}: c e^{h(\eta)} \eta: \bar{\eta}: 1\right]$ using $\eta \in \mathbb{C P}^{1}$ and $c \in \mathrm{U}(1)$, then such an $s \in S^{1}$ is characterized by the equation $e^{2 H_{+}+H_{0}+i s} \Phi=c e^{h(\eta)}$, so $s$ is unique, and $s=s(t, \lambda)$ is smooth. Hence we obtain a smooth section $\Sigma_{\pi(z)} \rightarrow \mathcal{M}:(t, \lambda) \rightarrow(s(t, \lambda), t, \lambda)$ of which the image is $\mathfrak{S}_{z} \cap \mathcal{M}$. Hence $\mathfrak{S}_{z} \cap \mathcal{M}$ is diffeomorphic to $\Sigma_{\pi(z)} \cong \mathbb{R} \times S^{1}$.

Now we notice the disks $\left\{\mathfrak{D}_{\xi}\right\}_{\xi \in\left(S_{+} \sqcup S_{-}\right)}$. Obviously, there are just two disks in this family satisfying $z \in \partial \mathfrak{D}_{\xi}$. Hence $\mathfrak{S}_{z}$ is the natural compactification of $\mathbb{R} \times S^{1}$ with two extra points, so $\mathfrak{S}_{z}$ is homeomorphic to $S^{2}$.

Finally we check the smoothness of $\mathfrak{S}_{z}$. Let us put $\widetilde{\mathfrak{S}}_{z}:=\hat{\mathfrak{f}}^{-1}(z)$. By a similar argument as above, $\widetilde{\mathfrak{S}}_{z} \cap \mathcal{Z}_{\mathbb{R}}$ is an embedded $\mathbb{R} \times S^{1}$ in $\mathcal{Z}_{\mathbb{R}}$, and $\widetilde{\mathfrak{S}}_{z}$ is the natural compactification of $\widetilde{\mathfrak{S}}_{z} \cap \mathcal{Z}_{\mathbb{R}}$ with two extra points. Recall that $\widetilde{\mathfrak{S}}_{z} \cap \mathcal{Z}_{\mathbb{R}}=\mathfrak{f}^{-1}(z)$ is an integral surface of the distribution $\mathcal{D} \mid \mathcal{Z}_{\mathbb{R}}$ by Proposition 7.6. Then, the whole of $\widetilde{\mathfrak{S}}_{z}$ is an integral surface of $\mathcal{D}$ by continuity. Since the distribution $\mathcal{D}$ is smooth, $\widetilde{\mathfrak{S}}_{z}$ is smoothly embedded in $S^{2}$. Hence $\mathfrak{S}_{z}=p\left(\widetilde{\mathfrak{S}}_{z}\right)$ is also smoothly embedded in $S^{2}$. 
Proposition 7.9. The map $\hat{\mathfrak{f}}:\left(\hat{\mathcal{Z}}_{+}, \hat{\mathcal{Z}}_{\mathbb{R}}\right) \rightarrow\left(\mathbb{C P}^{3}, P_{h}\right)$ satisfies the following conditions:

(1) each fiber of $\hat{\mathfrak{f}}: \hat{\mathcal{Z}}_{\mathbb{R}} \rightarrow P_{h}$ is an integral surface of the distribution $\mathcal{D}$,

(2) $\hat{\mathfrak{f}}:\left(\hat{\mathcal{Z}}_{+} \backslash \hat{\mathcal{Z}}_{\mathbb{R}}\right) \rightarrow\left(\mathbb{C P}^{3} \backslash P_{h}\right)$ is biholomorphic.

Proof. We already showed statement (1) in the proof of Proposition 7.8 The holomorphicity of $\hat{\mathfrak{f}}:\left(\hat{\mathcal{Z}}_{+} \backslash \hat{\mathcal{Z}}_{\mathbb{R}}\right) \rightarrow\left(\mathbb{C P}^{3} \backslash P_{h}\right)$ is deduced from (1) and the fiberwise holomorphicity of $\hat{\mathfrak{f}}$. Further, $\hat{\mathfrak{f}}:\left(\hat{\mathcal{Z}}_{+} \backslash \hat{\mathcal{Z}}_{\mathbb{R}}\right) \rightarrow\left(\mathbb{C P}^{3} \backslash P_{h}\right)$ is bijective by (3) of Proposition 7.7 hence (2) follows.

Corollary 7.10. Let $(V, A)$ be an admissible monopole. Then the anti-self-dual metric on $S^{2} \times S^{2}$ induced by $(V, A)$ is Zollfrei.

Proof. As proved in [19] (Theorem 5.14), an anti-self-dual 4-manifold $\left(S^{2} \times S^{2},[g]\right)$ is Zollfrei if and only if every $\alpha$-surface is an embedded $S^{2} \subset S^{2} \times S^{2}$. (Here we are taking the opposite orientation to [19].) In our situation, every $\alpha$-surface is given as the image of an integral surface of $\mathcal{D}$ by $p: \hat{\mathcal{Z}}_{\mathbb{R}} \rightarrow S^{2} \times S^{2}$. Hence every $\alpha$-surface is written as $\mathfrak{S}_{z}=\hat{p}\left(\hat{\mathfrak{f}}^{-1}(z)\right)$ for some $z \in P_{h}$ by Proposition 7.9. Since $\mathfrak{S}_{z}$ is an embedded $S^{2} \subset S^{2} \times S^{2}$ by Proposition 7.8 , the statement follows.

The proof of the main theorem (Theorem 7.1) is already finished. Actually, the Zollfrei condition of the considered metric is proved in Corollary 7.10, and Propositions 7.7 and 7.9 mean that the pair $\left(\mathbb{C P}^{3}, P_{h}\right)$ is the very LeBrun-Mason twistor space.

\section{CONCLUding REMARKS}

Regularity. In this article, we assumed the smoothness of functions, embeddings, and so on. In the previous articles [18, 19, 24, however, we can construct the twistor correspondences of low regularities. Similar to these previous works, the argument in this article should be strengthened to that of low regularities. Actually the integral transforms $R$ and $Q$ are defined even for non-differentiable functions, and hyperbolic partial differential equations admit solutions of low regularities or distribution solutions in general. Thus the notion of a self-dual Zollfrei metric might be generalized to, for example, a non-differentiable class. In fact, infinitely many examples of 'self-dual Zollfrei metrics with singularity' are already obtained in [22].

Degeneration. We introduced the notion of admissible monopoles in Section 4 and showed that the corresponding admissible deformation $\mathbb{R P}^{3}$ in $\mathbb{C P}^{3}$ has nice properties and the LeBrun-Mason correspondence works well (Theorem 7.1). On the other hand, in the non-admissible case, the deformation of $\mathbb{R} \mathbb{P}^{3}$ in $\mathbb{C P}^{3}$ has an unexpected property (Proposition 7.3). Even in the non-admissible case, however, we can get the family of holomorphic disks parametrized by $S^{2} \times S^{2}$ (Proposition 7.2). Then the natural question is:

- Is there any natural structure on the parameter space of the holomorphic disks for the non-admissible case?

In particular, it would be interesting to study the process of the degeneration which occurs in the deformation from an admissible case to a non-admissible case.

Deformation of $\mathbf{S}_{1}^{\mathbf{3}}$. The argument in this article is based on the identification between the de Sitter space $S_{1}^{3}$ and the space of oriented small circles on the twosphere $\mathbb{S}^{2}$, which arises from the LeBrun-Mason correspondence for Einstein-Weyl 
structures [20, 24]. By the result in [20, 24], if we deform the twistor space from $\left(W, W_{\mathbb{R}}\right)=\left(\mathbb{C P}^{1} \times \mathbb{C P}^{1}, \mathbb{C P}^{1}\right)$ to $(W, P)$, we obtain an Einstein-Weyl structure on $\mathbb{R} \times S^{2}$ of indefinite signature. In this construction, $\mathbb{R} \times S^{2}$ is identified with the space of oriented circles embedded in $P \simeq \mathbb{C P}^{1}$. So it is natural to expect the generalization of our story to such deformed situations. If it is successful, we will obtain various significant objects: general solutions of the wave equations on $\mathbb{R} \times S^{2}$, descriptions of more general self-dual Zollfrei metrics, its LeBrun-Mason twistor spaces, and so on.

\section{Appendix A. The BiJectivity of $\mathcal{Q}$}

We give a proof of the bijectivity of the transform $\mathcal{Q}: C_{\text {odd }}^{\infty}\left(\mathbb{S}^{2}\right) \rightarrow C_{\text {odd }}^{\infty}\left(\mathbb{S}^{2}\right)$ by a method similar to Guillemin's [7. Let $\hat{\mathscr{H}}^{k}$ be the space of homogeneous harmonic polynomials of degree $k$ on $\mathbb{R}^{3}$ and let $\mathscr{H}^{k}=\left\{\left.P\right|_{\mathbb{S}^{2}} \in L^{2}\left(\mathbb{S}^{2}\right) \mid P \in \hat{\mathscr{H}}\right\}$. We notice the following fact.

Theorem A.1. The group $\mathrm{SO}(3)$ acts irreducibly on $\mathscr{H}^{k}$ and the representations on $\mathscr{H}^{k}$ and $\mathscr{H}^{l}$ are inequivalent if $k \neq l$. Moreover, we have the decomposition

$$
L^{2}\left(\mathbb{S}^{2}\right) \cong \bigoplus_{k} \mathscr{H}^{k}
$$

as a direct sum of Hilbert spaces.

Since $\mathcal{Q}$ maps $L^{2}\left(\mathbb{S}^{2}\right)$ to itself and commutes with the $S O(3)$-action, so $\mathcal{Q}$ is diagonalized with respect to the decomposition (A.1). Let us denote the eigenvalues of $\mathcal{Q}$ on $\mathscr{H}^{k}$ by $c(k) \in \mathbb{R}$, that is,

$$
\mathcal{Q} h=c(k) \cdot h \quad \text { for } h \in \mathscr{H}^{k} .
$$

\section{Proposition A.2.}

$$
c(k)=\left\{\begin{array}{lll}
1 & k=0, \\
0 & k=2 m \quad(m=1,2, \cdots), \\
(-1)^{m} \frac{4 \pi}{2 m+1} \cdot \frac{1 \cdot 3 \cdot 5 \cdots(2 m+1)}{2 \cdot 4 \cdot 6 \cdots(2 m+2)} & k=2 m+1 \quad(m=0,1, \cdots) .
\end{array}\right.
$$

Proof. Since $\mathcal{Q}(1)=1$ by definition, we obtain $c(0)=1$. On the other hand, since $C_{\text {even } *}^{\infty}\left(\mathbb{S}^{2}\right)$ is annihilated by $\mathcal{Q}$, we obtain $c(2 m)=0$ for $m>0$.

Suppose $k=2 m+1$. Let us choose a harmonic polynomial $P(x, y, z) \in \hat{\mathscr{H}}^{k}$ so that it does not depend on the $z$ variable. Then $P$ is written as

$$
P(x, y, z)=a_{2 m+1} x^{2 m+1}+a_{2 m} x^{2 m} y+\cdots+a_{0} y^{2 m+1} .
$$

Since $P$ is harmonic, the equation $\left(\partial_{x}^{2}+\partial_{y}^{2}\right) P=0$ holds. Hence we obtain

$$
\begin{aligned}
& a_{2 j+1}=-\frac{(2 m-2 j+2)(2 m-2 j+1)}{(2 j+1) \cdot 2 j} a_{2 j-1} \quad(j=1,2, \cdots, m) \\
& \text { or } \quad a_{2 m+1}=(-1)^{m} \frac{2 m \cdot(2 m-1) \cdots 2 \cdot 1}{(2 m+1) \cdot 2 m \cdots 3 \cdot 2} a_{1}=\frac{(-1)^{m}}{2 m+1} a_{1} .
\end{aligned}
$$

Now we have $(\mathcal{Q P})(1,0,0)=c(2 m+1) P(1,0,0)=c(2 m+1) a_{2 m+1}$. On the other hand, by definition,

$$
(\mathcal{Q} P)(1,0,0)=\int_{\Omega} P(x, y) \omega_{\mathbb{S}^{2}}, \quad \text { where } \Omega=\left\{(x, y, z) \in \mathbb{S}^{2} \mid x>0\right\} .
$$


Let us use the coordinate $(\theta, \varphi)$ so that

$$
(x, y, z)=(\sin \theta \cos \varphi, \sin \theta \sin \varphi, \cos \theta), \quad \Omega=\left\{0 \leq \theta \leq \pi,-\frac{\pi}{2} \leq \varphi \leq \frac{\pi}{2}\right\} .
$$

Then, by $\omega_{\mathbb{S}^{2}}=\sin \theta d \theta d \varphi$,

$$
\begin{aligned}
(\mathcal{Q} P)(1,0,0) & =\int_{\Omega} P(\sin \theta \cos \varphi, \sin \theta \sin \varphi) \sin \theta d \theta d \varphi \\
& =\sum_{l=0}^{2 m+1} a_{l}\left(\int_{0}^{\pi}(\sin \theta)^{2 m+2} d \theta\right)\left(\int_{-\frac{\pi}{2}}^{\frac{\pi}{2}}(\cos \varphi)^{l}(\sin \varphi)^{2 m-l+1} d \varphi\right) \\
& =2\left(\int_{0}^{\pi}(\sin \theta)^{2 m+2} d \theta\right) \sum_{j=0}^{m} a_{2 j+1}\left(\int_{0}^{\frac{\pi}{2}}(\cos \varphi)^{2 j+1}(\sin \varphi)^{2 m-2 j} d \varphi\right) .
\end{aligned}
$$

By a usual trick, which is also explained in [7, we obtain

$$
\int_{0}^{\pi}(\sin \theta)^{2 m+2} d \theta=2 \pi \frac{1 \cdot 3 \cdot 5 \cdots(2 m+1)}{2 \cdot 4 \cdot 6 \cdots(2 m+2)} .
$$

On the other hand, if we put

$$
B(j)=\int_{0}^{\frac{\pi}{2}}(\cos \varphi)^{2 j+1}(\sin \varphi)^{2 m-2 j} d \varphi,
$$

then for $j>0$,

$$
\begin{aligned}
B(j) & =\left[(\cos \varphi)^{2 j} \cdot \frac{(\sin \varphi)^{2 m-2 j+1}}{2 m-2 j+1}\right]_{0}^{\frac{\pi}{2}}-\int_{0}^{\frac{\pi}{2}} \frac{\partial}{\partial \varphi}\left((\cos \varphi)^{2 j}\right) \cdot \frac{(\sin \varphi)^{2 m-2 j+1}}{2 m-2 j+1} d \varphi \\
& =\frac{2 j}{2 m-2 j+1} B(j-1) .
\end{aligned}
$$

Hence, combining with (A.2), we obtain

$$
\begin{gathered}
a_{2 j+1} B(j)=-\frac{2 m-2 j+2}{2 j+1} a_{2 j-1} B(j-1) \\
\text { or } \quad a_{2 j+1} B(j)=\frac{2 m-2 j}{2 m+1} a_{2 j+1} B(j)-\frac{2 m-2 j-2}{2 m+1} a_{2 j-1} B(j-1) .
\end{gathered}
$$

If we take a sum of (A.6) for $j=1,2, \cdots, m$, then we obtain

$$
\sum_{j=1}^{m} a_{2 j+1} B(j)=-\frac{2 m}{2 m+1} a_{1} B(0) .
$$

Thus

$$
\sum_{j=0}^{m} a_{2 j+1} B(j)=\frac{1}{2 m+1} a_{1} B(0)=\frac{(-1)^{m}}{2 m+1} a_{2 m+1} .
$$

By (A.4) and (A.7),

$$
(\mathcal{Q P})(1,0,0)=c(2 m+1) a_{2 m+1}=(-1)^{m} \frac{4 \pi}{2 m+1} \cdot \frac{1 \cdot 3 \cdot 5 \cdots(2 m+1)}{2 \cdot 4 \cdot 6 \cdots(2 m+2)} a_{2 m+1} .
$$

Since $a_{2 m+1} \neq 0$, we obtain the required formula. 
We denote the degree $s$ Sobolev space over $\mathbb{S}^{2}$ by $H^{s}$, and put $H_{\text {odd }}^{s}:=H^{s} \cap$ $L_{\text {odd }}^{2}\left(\mathbb{S}^{2}\right)$. Let us define a norm on $H^{s}$ by

$$
|h|_{s}=k^{s}|h|_{L^{2}} \quad \text { for } \quad h \in \mathscr{H}^{k} .
$$

Then, as explained in [7, this norm is equivalent to the usual $H^{s}$ norm.

Proposition A.3. There exists a constant $c>1$ independent of $s$ such that

$$
\frac{1}{c}|h|_{s} \leq|\mathcal{Q} h|_{s+\frac{3}{2}} \leq c|h|_{s}
$$

for all $s$ and $h \in H_{\text {odd }}^{s}$. Hence $\mathcal{Q}$ defines a bijection $H_{\text {odd }}^{s} \rightarrow H_{\text {odd }}^{s+\frac{3}{2}}$.

Proof. Similarly to [7, we consider the formula

$$
\pi^{\frac{1}{2}}=\lim _{k \rightarrow \infty} k^{-\frac{1}{2}} \frac{2 \cdot 4 \cdot 6 \cdots 2 m}{1 \cdot 3 \cdot 5 \cdots(2 m-1)} .
$$

By Proposition A.2, we get

$$
c(2 m-1) \sim(-1)^{k} 2 \pi^{\frac{1}{2}} k^{-\frac{3}{2}} .
$$

So the statement follows.

By Proposition A.3 and the Sobolev embedding theorem, we obtain the following.

Theorem A.4. The transform $\mathcal{Q}: C_{\text {odd }}^{\infty}\left(\mathbb{S}^{2}\right) \rightarrow C_{\text {odd }}^{\infty}\left(\mathbb{S}^{2}\right)$ is bijective.

\section{Appendix B. Formulas on $S_{1}^{3}$}

In Section 6. equation (6.5), we introduced a local orthonormal frame $\left\{\underline{E}_{1}, \underline{E}_{2}\right.$, $\left.\underline{E}_{3}\right\}$ of the tangent bundle $T S_{1}^{3}$ on the open set $\underline{U}=\{(t, \lambda) \mid \lambda \neq \infty\} \subset S_{1}^{3}$. Here we show several formulas concerning this frame. All these formulas are deduced by direct calculations.

The connection form $\omega$ of the Levi-Civita connection for $g_{S_{1}^{3}}$ and its curvature form $K$ are

$$
\begin{gathered}
\omega=\left(\begin{array}{ccc}
0 & \omega_{2}^{1} & \omega_{3}^{1} \\
\omega_{2}^{1} & 0 & \omega_{3}^{2} \\
\omega_{3}^{1} & -\omega_{3}^{2} & 0
\end{array}\right), \quad \underline{\omega}_{2}^{1}=\tanh t \underline{E}^{2}, \quad \underline{\omega}_{3}^{2}=\frac{1}{\cosh t}\left(-\operatorname{Im} \lambda \underline{\underline{E}}^{2}+\operatorname{Re} \lambda \underline{E}^{3}\right), \\
K=\left(\begin{array}{ccc}
0 & \underline{E}^{1} \wedge \underline{E}^{2} & \underline{E^{1}} \wedge \underline{E}^{3} \\
\underline{E^{1}} \wedge \underline{E}^{2} & 0 & \underline{E}^{2} \wedge \underline{E}^{3} \\
\underline{E}^{1} \wedge \underline{E}^{3} & \underline{E}^{3} \wedge \underline{E}^{2} & 0
\end{array}\right) .
\end{gathered}
$$

Let $\underline{\mathfrak{m}}_{1}(\zeta)$ and $\underline{\mathfrak{m}}_{2}(\zeta)$ be the vector fields on $\underline{U}$ defined by (5.3). By the lifting formula (5.6), the tautological lifts $\underline{\mathfrak{\mathfrak { m }}}_{1}$ and $\underline{\mathfrak{\mathfrak { m }}}_{2}$ on $\mathcal{W}_{\mathbb{R}}$ (or on $\mathcal{W}_{+}$) are written as (B.3)

$$
\left\{\begin{array}{ll}
\underline{\tilde{\mathfrak{m}}}_{1}=\underline{\mathfrak{m}}_{1}+\gamma_{1} \partial_{\zeta}, & \gamma_{1}=\Psi \cdot(-\operatorname{Im} \lambda+\zeta \operatorname{Re} \lambda-\zeta \sinh t), \\
\underline{\mathfrak{\mathfrak { m }}}_{2}=\underline{\mathfrak{m}}_{2}+\gamma_{2} \partial_{\zeta}, & \gamma_{2}=\Psi \cdot(-\zeta \operatorname{Im} \lambda-\operatorname{Re} \lambda-\sinh t),
\end{array}:=\frac{1+\zeta^{2}}{2 \cosh t} .\right.
$$

If we change the fiber coordinate by $\zeta=i \frac{1-\omega}{1+\omega}$, we obtain

$$
\left\{\begin{array}{l}
(1+\omega) \underline{\mathfrak{m}}_{1}=l_{1}+\omega \bar{l}_{1}+\left(\delta_{1}-\omega \bar{\delta}_{1}\right) \omega \partial_{\omega}, \\
(1+\omega) \underline{\mathfrak{\mathfrak { m }}}_{2}=l_{2}+\omega \bar{l}_{2}+\left(\delta_{2}-\omega \bar{\delta}_{2}\right) \omega \partial_{\omega},
\end{array}\right.
$$


where

$$
\begin{cases}l_{1}=-\underline{E}_{1}+\underline{E}_{2}+i \underline{E}_{3}, & \delta_{1}=-\frac{\lambda}{\cosh t}+\tanh t, \\ l_{2}=i \underline{E}_{1}+i \underline{E}_{2}-\underline{E}_{3}, & \delta_{2}=-\frac{i \lambda}{\cosh t}-i \tanh t .\end{cases}
$$

\section{ACKNOWLEDGMENTS}

The author would like to thank Hiroyuki Kamada for helpful conversations and for providing many useful references, and Mikio Furuta for helpful discussions. The author would also like to thank Toshio Oshima for introducing previous works on representation theory.

\section{REFERENCES}

[1] M. F. Atiyah, N. Hitchin, I. M. Singer: Self-duality in Four-dimensional Riemannian Geometry, Proc. R. Soc. Lond. A.362 (1978) 425-461. MR506229 (80d:53023)

[2] T. N. Bailey, M. G. Eastwood, A. R. Gover, L. J. Mason: The Funk transform as a Penrose transform, Math. Proc. Cambridge Philos. Soc. 125, no.1 (1999) 67-81. MR.1645521 (99i:32037)

[3] D. M. J. Calderbank: Selfdual 4-manifolds, projective surfaces, and the Dunajski-West construction, e-print math.DG/0606754 (2006)

[4] R. Courant, D. Hilbert: Methods of Mathematical Physics, vol. 2, Wiley-Interscience, New York, 1962. MR0140802 (25:4216)

[5] M. Dunajski, S. West: Anti-self-dual conformal structures with null Killing vectors from projective structures, Comm. Math. Phys. 272, no.1 (2007) 85-118. MR2291803 (2008g:53052)

[6] P. Funk: Über eine geometrische Anwendung der Abelshen Integral-gleichung, Math. Ann. 77 (1916) 129-135.

[7] V. Guillemin: The Radon Transform on Zoll Surfaces, Advances in Math. 22 (1976) 85-119. MR0426063 (54:14009)

[8] V. Guillemin: Cosmology in (2+1)-dimensions, cyclic models, and deformations of $M_{2,1}$, Annals of Mathematics Studies, 121, Princeton University Press, Princeton (1989). MR 999388 (91k:58140)

[9] S. Helgason: The Radon Transform (Second Edition), Progress in Mathematics, vol.5, Birkhäuser (1999). MR1723736 (2000m:44003)

[10] K. Hiraoka, S. Matsumoto, K. Okamoto: Eigenfunctions of the Laplacian on a Real Hyperboloid of One Sheet, Hiroshima Math. J. 7 (1977) 855-864. MR0622203 (58:29861)

[11] N. J. Hitchin: Complex manifolds and Einstein's equations, Twistor Geometry and NonLinear Systems, Lecture Notes in Mathematics vol. 970, Springer, Berlin-New York, 1982. MR0699802 (84i:32041)

[12] P. E. Jones, K. P. Tod: Minitwistor spaces and Einstein-Weyl spaces, Class. Quantum Grav. 2 (1985) 565-577. MR795102 (87b:53115)

[13] H. Kamada: Self-dual Kähler metrics of neutral signature on complex surfaces, Tohoku Mathematical Publications 24, Sendai: Tohoku University (2002). MR.1938369 (2004f:53050)

[14] H. Kamada: Compact Scalar-flat Indefinite Kähler Surfaces with Hamiltonian S $^{1}$-Symmetry, Comm. Math. Phys. 254 (2005) 23-44. MR2116737 (2006g:32025)

[15] V. Kotecha, R. S. Ward: Integrable Yang-Mills-Higgs equations in three-dimensional de Sitter space-time, J. Math. Phys. Vol. 42, Num. 3 (2000) 1018-1025. MR.1814433 (2002a:37104)

[16] C. LeBrun: Explicit self-dual metrics on $\mathbb{C P}_{2} \sharp \cdots \sharp \mathbb{C P}_{2}$, J. Diff. Geom. 34 (1991) 223-253. MR:1114461 (92g:53040)

[17] C. LeBrun: Twistors, Holomorphic Disks, and Riemann Surfaces with Boundary, Perspectives in Riemannian geometry, CRM Proc. Lecture Notes, 40, Amer. Math. Soc. Providence, RI, (2006) pp. 209-221. MR2237111 (2007g:53051)

[18] C. LeBrun, L. J. Mason: Zoll Manifolds and complex surfaces, J. Diff. Geom. 61, (2002) 453-535. MR1979367 (2004d:53043)

[19] C. LeBrun, L. J. Mason: Nonlinear Gravitons, Null Geodesics, and Holomorphic Disks, Duke Math. J. 136, no.2 (2007) 205-273. MR.2286630 (2008d:53058)

[20] C. LeBrun, L. J. Mason: The Einstein-Weyl Equations, Scattering Maps, and Holomorphic Disks, Math. Res. Lett. 16 (2009) 291-301. MR2496745 
[21] L. J. Mason: Global anti-self-dual Yang-Mills fields in split signature and their scattering, J. Reine Angew. Math. 597, (2006), 105-133. MR2264316 (2008a:53018)

[22] F. Nakata: Singular self-dual Zollfrei metrics and twistor correspondence, J. Geom. Phys. 57, no.6 (2007) 1477-1498. MR2294508 (2008e:53080)

[23] F. Nakata: Self-dual Zollfrei conformal structures with $\alpha$-surface foliation, J. Geom. Phys. 57, no.10 (2007) 2077-2097. MR2348280(2008e:53079)

[24] F. Nakata: A construction of Einstein-Weyl spaces via LeBrun-Mason type twistor correspondence, Comm. Math. Phys. 289 (2009) 663-699. MR2506766 (2010j:53079)

[25] T. Oshima, J. Sekiguchi: Eigenspaces of Invariant Differential Operators on an Affine Symmetric Space, Inventiones Math. 57 (1980) 1-81. MR564184 (81k:43014)

[26] R. Penrose: Nonlinear gravitons and curved twistor theory, Gen. Rel. Grav. 7 (1976) 31-52. MR0439004 (55:11905)

[27] K. P. Tod: Indefinite conformally-ASD metric on $S^{2} \times S^{2}$ : Further advances in twistor theory. Vol.III. Chapman \& Hall/CRC (2001) pp.61-63, reprinted from Twistor Newsletter 36 (1993).

Department of Mathematics, Graduate School of Science and Engineering, Tokyo Institute of Technology, 2-12-1, O-OKayama, Meguro, 152-8551, Japan

E-mail address: nakata@math.titech.ac.jp

Current address: Faculty of Human Development and Culture, Fukushima University, 1, Kanayagawa, Fukushima, 960-1296, Japan

E-mail address: fnakata@educ.fukushima-u.ac.jp 